\title{
CRITĖRE POUR L'INTÉGRALITÉ DES COEFFICIENTS DE TAYLOR DES APPLICATIONS MIROIR
}

\author{
E. DELAYGUE
}

\begin{abstract}
RÉSUmÉ. Nous donnons une condition nécessaire et suffisante pour que les coefficients de Taylor de séries de la forme $q(z):=z \exp (G(z) / F(z))$ soient entiers, où $F(z)$ et $G(z)+\log (z) F(z)$ sont des solutions particulières de certaines équations différentielles hypergéométriques généralisées. Ce critère est basé sur les propriétés analytiques de l'application de Landau (classiquement associée aux suites de quotients de factorielles) et il généralise les résultats de Krattenthaler-Rivoal dans On the integrality of the Taylor coefficients of mirror maps, Duke Math. J. à paraître. Pour démontrer ce critère, nous généralisons entre autres un théorème de Dwork concernant les congruences formelles entre séries formelles dans $O n$ p-adic differential equations $I V$ : generalized hypergeometric functions as p-adic functions in one variable, Annales scientifiques de l'E.N.S., ce dernier ne suffisant pas dans notre cas.
\end{abstract}

\section{INTRODUCTION}

1.1. Applications miroir. Les applications miroir sont des séries entières $z(q)$ inverses pour la composition de séries entières $q(z):=z \exp (G(z) / F(z))$, où $F(z)$ et $G(z)+\log (z) F(z)$ sont des solutions particulières de certaines équations différentielles hypergéométriques généralisées. Elles dépendent de nombreux paramètres et, dans les cas les plus simples, elles s'identifient à des formes modulaires classiques définies sur différents sous-groupes de congruence de $S L_{2}(\mathbb{Z})$. Ces dernières apparaissant naturellement dans la théorie de Schwarz des fonctions hypergéométriques de Gauss (voir [12]). Les applications miroir ont connu un regain d'intérêt à la fin des années 1980, à la suite de travaux en théorie des cordes qui ont amené des physiciens à étudier des Calabi-Yau threefolds et à construire leur variété miroir. En particulier, cette construction est associée à une application miroir dont les coefficients de Taylor permettent dans certains cas de compter les courbes rationnelles sur les Calabi-Yau threefolds (voir [1] par exemple).

Sur de nombreux exemples en symétrie miroir, il a été observé que les coefficients de Taylor des applications miroir associées sont entiers. Cette observation a été démontrée dans de nombreux cas (voir un peu plus bas dans l'introduction) et le sujet de cet article est la démonstration d'un critère d'intégralité des coefficients de Taylor des applications miroir issues de certaines équations différentielles hypergéométriques généralisées. Ces équations s'interprètent souvent comme des équations de Picard-Fuchs de familles à un paramètre d'intersections complètes de Calabi-Yau dans des espaces projectifs à poids (voir [1]). Dans les cas où les applications miroir sont des formes modulaires classiques, l'intégralité de leurs coefficients de Taylor est conséquence des théorèmes de structure classiques des algèbres de formes modulaires. Néanmoins, dans le cadre plus général de cet article, la modularité au sens usuel disparait et on doit faire appel à des techniques différentes pour aborder ces problèmes d'intégralité.

Dans la suite, si e $:=\left(e_{1}, \ldots, e_{q_{1}}\right)$ et $\mathbf{f}:=\left(f_{1}, \ldots, f_{q_{2}}\right)$ sont deux suites d'entiers positifs, on note $|\mathbf{e}|:=\sum_{i=1}^{q_{1}} e_{i},|\mathbf{f}|:=\sum_{j=1}^{q_{2}} f_{j}$ et $\mathcal{Q}_{(\mathbf{e}, \mathbf{f})}(n):=\frac{\left(e_{1} n\right) ! \cdots\left(e_{q_{1}} n\right) !}{\left(f_{1} n\right) ! \cdots\left(f_{q_{2}}\right) !}$, où $n \geq 0$. On définit les séries entières

$$
F_{(\mathbf{e}, \mathbf{f})}(z):=\sum_{n=0}^{\infty} \frac{\left(e_{1} n\right) ! \cdots\left(e_{q_{1}} n\right) !}{\left(f_{1} n\right) ! \cdots\left(f_{q_{2}} n\right) !} z^{n}
$$




$$
G_{(\mathbf{e}, \mathbf{f})}(z):=\sum_{n=1}^{\infty} \frac{\left(e_{1} n\right) ! \cdots\left(e_{q_{1}} n\right) !}{\left(f_{1} n\right) ! \cdots\left(f_{q_{2}} n\right) !}\left(\sum_{i=1}^{q_{1}} e_{i} H_{e_{i} n}-\sum_{j=1}^{q_{2}} f_{j} H_{f_{j} n}\right) z^{n}
$$

où $H_{n}:=\sum_{i=1}^{n} \frac{1}{i}$ est le $n$-ième nombre harmonique. La série $F_{(\mathbf{e}, \mathbf{f})}(z)$ est une série hypergéométrique généralisée (1) et est donc solution d'une équation différentielle fuchsienne. Dans certains cas que l'on étudiera (voir la fin du paragraphe 6.2), on obtient, via la méthode de Frobenius (voir [12]), une base des solutions de cette équation différentielle avec au plus des singularités logarithmiques à l'origine, dont $F_{(\mathbf{e}, \mathbf{f})}(z)$ et $G_{(\mathbf{e}, \mathbf{f})}(z)+\log (z) F_{(\mathbf{e}, \mathbf{f})}(z)$.

Dans le contexte de la symétrie miroir, la fonction $q_{(\mathbf{e}, \mathbf{f})}(z):=z \exp \left(G_{(\mathbf{e}, \mathbf{f})}(z) / F_{(\mathbf{e}, \mathbf{f})}(z)\right)$ est une coordonnée canonique et son inverse pour la composition $z(q)$ est une application miroir. Le but de cet article est d'établir une condition nécessaire et suffisante pour l'intégralité des coefficients des applications miroir $z(q)$, c'est-à-dire de déterminer sous quelles conditions on a $z(q) \in \mathbb{Z}[[q]]$. Dans le contexte de théorie des nombres de cet article, l'application miroir $z(q)$ et la coordonnée canonique correspondante $q(z)$ jouent exactement le même rôle car $q(z) \in z \mathbb{Z}[[z]]$ si et seulement si $z(q) \in q \mathbb{Z}[[q]]$ (voir $[9$, Introduction]). On formulera donc le critère pour $q(z)$ uniquement mais il vaut aussi pour $z(q)$.

1.2. Énoncé du critère. Avant d'énoncer le critère d'intégralité des coefficients de Taylor de $q(z)$, nous rappelons la définition de l'application de Landau associée à un quotient de factorielles. Étant données $\mathbf{e}:=\left(e_{1}, \ldots, e_{q_{1}}\right)$ et $\mathbf{f}:=\left(f_{1}, \ldots, f_{q_{2}}\right)$ deux suites d'entiers positifs, on note $\Delta_{(\mathbf{e}, \mathbf{f})}$ la fonction de Landau associée à $\mathcal{Q}_{(\mathbf{e}, \mathbf{f})}$ définie, pour tout $x \in \mathbb{R}$, par

$$
\Delta_{(\mathbf{e}, \mathbf{f})}(x):=\sum_{i=1}^{q_{1}}\left\lfloor e_{i} x\right\rfloor-\sum_{j=1}^{q_{2}}\left\lfloor f_{j} x\right\rfloor
$$

où $\lfloor$.$\rfloor désigne la fonction partie entière. La fonction \Delta_{(\mathbf{e}, \mathbf{f})}$ est constante par morceaux. Notons $M_{(\mathbf{e}, \mathbf{f})}:=\max \left\{e_{1}, \ldots, e_{q_{1}}, f_{1}, \ldots, f_{q_{2}}\right\}$. La fonction $\Delta_{(\mathbf{e}, \mathbf{f})}$ est nulle sur $\left[0,1 / M_{(\mathbf{e}, \mathbf{f})}[\right.$ car $0 \leq$ $e_{i} / M_{(\mathbf{e}, \mathbf{f})} \leq 1$ et $0 \leq f_{j} / M_{(\mathbf{e}, \mathbf{f})} \leq 1$. La proposition suivante montre que la fonction de Landau permet de caractériser les suites e et $\mathbf{f}$ telles que, pour tout $n \in \mathbb{N}, \mathcal{Q}_{(\mathbf{e}, \mathbf{f})}(n)$ est entier.

Critère de Landau (Landau, Bober). Soit $\boldsymbol{e}$ et $\boldsymbol{f}$ deux suites d'entiers strictement positifs disjointes. On a la dichotomie suivante.

(i) Si, pour tout $x \in[0,1]$, on a $\Delta_{(\boldsymbol{e}, \boldsymbol{f})}(x) \geq 0$, alors, pour tout $n \in \mathbb{N}$, on a $\mathcal{Q}_{(\boldsymbol{e}, \boldsymbol{f})}(n) \in \mathbb{N}$.

(ii) S'il existe un $x \in[0,1]$ tel que $\Delta_{(\boldsymbol{e}, \boldsymbol{f})}(x) \leq-1$, alors il n'existe qu'un nombre fini de nombres premiers $p$ tels que tous les termes de la suite $\mathcal{Q}_{(\boldsymbol{e}, \boldsymbol{f})}$ soient dans $\mathbb{Z}_{p}$.

Remarque. Le point $(i)$ est dû à Landau qui énonce une condition nécessaire et suffisante dans [6], et le point (ii) est dû à Bober (voir [2]).

Dans la littérature, on peut distinguer trois résultats établissant l'intégralité des coefficients de Taylor d'applications miroir appartenant à des ensembles de plus en plus grand.

Le premier résultat a été démontré par Lian et Yau dans [8], dans le cas où $\mathcal{Q}_{(\mathbf{e}, \mathbf{f})}(n)=\frac{(p n) !}{(n !)^{p}}$, où $p$ est un nombre premier.

Ce résultat a été généralisé par Zudilin dans [13]. Soit $N \in \mathbb{N}$ et $N=p_{1}^{a_{1}} \ldots p_{\ell}^{a_{\ell}}$ sa décomposition en facteurs premiers. On note $A_{N}$ et $B_{N}$ les multi-ensembles (2) définis par $A_{N}:=$ $\left\{N, \frac{N}{p_{j_{1}} p_{j_{2}}}, \frac{N}{p_{j_{1}} p_{j_{2}} p_{j_{3}} p_{j_{4}}}, \ldots\right\}_{1 \leq j_{1}<j_{2}<\cdots \leq \ell}$ et $B_{N}:=\left\{1, \ldots, 1, \frac{N}{p_{j_{1}}}, \frac{N}{p_{j_{1}} p_{j_{2}} p_{j_{3}}}, \ldots\right\}_{1 \leq j_{1}<j_{2}<\cdots \leq \ell}$, avec un nombre de 1 dans $B_{n}$ égal à $\varphi(N)$, où $\varphi$ est la fonction indicatrice d'Euler. Zudilin a montré que si e

\footnotetext{
${ }^{1}$ On caractérisera dans la proposition 2 de la partie 6 les fonctions hypergéométriques généralisées dont les coefficients peuvent se mettre sous forme de factorielles.

${ }^{2}$ Un multi-ensemble est un ensemble dans lequel on autorise les répétitions des éléments.
} 
est une suite constituée des éléments du multi-ensemble $\bigcup_{i=1}^{k} A_{N_{i}}$ et si $\mathbf{f}$ est une suite constituée des éléments du multi-ensemble $\bigcup_{i=1}^{k} B_{N_{i}}$, où les $N_{i}$ sont des entiers strictement positifs ayant le même ensemble de diviseurs premiers, alors l'application miroir associée à $(\mathbf{e}, \mathbf{f})$ a tous ses coefficients de Taylor entiers (3). Par exemple, on peut appliquer le théorème de Zudilin aux suites $\mathcal{Q}_{(\mathbf{e}, \mathbf{f})}(n)=\frac{(4 n) !}{(2 n) !(n !)^{2}}$ et $\mathcal{Q}_{(\mathbf{e}, \mathbf{f})}(n)=\frac{(6 n) !}{(3 n) !(2 n) ! n !}$, respectivement attachées aux choix des paramètres $k=1, N_{1}=4$ et $k=1, N_{1}=6$.

Enfin, Krattenthaler et Rivoal ont démontré la conjecture de Zudilin (voir [13, p. 605]).

Théorème (Krattenthaler-Rivoal, [5]). Soit $k \in \mathbb{N}, k \geq 1$, et $N_{1}, \ldots, N_{k}$ des entiers strictement positifs. Soit e la suite constituée des éléments du multi-ensemble $\bigcup_{i=1}^{k} A_{N_{i}}$ et $f$ la suite constituée des éléments du multi-ensemble $\bigcup_{i=1}^{k} B_{N_{i}}$. Alors l'application miroir associée à $(\boldsymbol{e}, \boldsymbol{f})$ a tous ses coefficients entiers.

Il s'avère que le cas traité par Krattenthaler et Rivoal correspond exactement aux quotients de factorielles dont l'application de Landau associée est croissante sur [0,1[ (4). On peut donc reformuler ce théorème en portant les conditions sur la fonction $\Delta_{(\mathbf{e}, \mathbf{f})}$.

Théorème bis (Krattenthaler-Rivoal, [5]). Soit e et $\boldsymbol{f}$ deux suites d'entiers strictement positifs disjointes vérifiant $|\boldsymbol{e}|=|\boldsymbol{f}|$. Si $\Delta_{(\boldsymbol{e}, \boldsymbol{f})}$ est croissante sur $\left[0,1\left[\right.\right.$, alors $q_{(\boldsymbol{e}, \boldsymbol{f})} \in z \mathbb{Z}[[z]]$.

Remarque. La croissance de $\Delta_{(\mathbf{e}, \mathbf{f})}$ sur $[0,1[$ implique sa positivité sur $[0,1]$ et donc, d'après le critère de Landau, la suite $\mathcal{Q}_{(\mathbf{e}, \mathbf{f})}$ est à termes entiers.

Le but de cet article est de démontrer le théorème suivant, qui caractérise complètement les applications miroir d'origine hypergéométrique (sous forme factorielle) ayant tous leur coefficients de Taylor entiers. Il contient les résultats des auteurs précédents.

Théorème 1. Soit $\boldsymbol{e}$ et $\boldsymbol{f}$ deux suites d'entiers strictement positifs disjointes, vérifiant $|\boldsymbol{e}|=|\boldsymbol{f}|$ et telles que $\mathcal{Q}_{(\boldsymbol{e}, \boldsymbol{f})}$ soit une suite à termes entiers (ce qui équivaut à $\Delta_{(\boldsymbol{e}, \boldsymbol{f})} \geq 0$ sur $[0,1]$ ). On a alors la dichotomie suivante.

(i) Si, pour tout $x \in\left[1 / M_{(\boldsymbol{e}, \boldsymbol{f})}, 1\left[\right.\right.$, on a $\Delta_{(\boldsymbol{e}, \boldsymbol{f})}(x) \geq 1$, alors $q_{(\boldsymbol{e}, \boldsymbol{f})}(z) \in z \mathbb{Z}[[z]]$.

(ii) S'il existe un $x \in\left[1 / M_{(\boldsymbol{e}, \boldsymbol{f})}, 1\left[\right.\right.$ tel que $\Delta_{(\boldsymbol{e}, \boldsymbol{f})}(x)=0$, alors il n'existe qu'un nombre fini de nombres premiers $p$ tels que $q_{(\boldsymbol{e}, \boldsymbol{f})}(z) \in z \mathbb{Z}_{p}[[z]]$.

Nous allons maintenant énoncer un critère pour l'intégralité des applications de type miroir $q_{L,(\mathbf{e}, \mathbf{f})}$ définies, pour tout entier $L \geq 1$, par $q_{L,(\mathbf{e}, \mathbf{f})}:=\exp \left(G_{L,(\mathbf{e}, \mathbf{f})}(z) / F_{(\mathbf{e}, \mathbf{f})}(z)\right)$, où $G_{L,(\mathbf{e}, \mathbf{f})}$ est la série formelle

$$
G_{L,(\mathbf{e}, \mathbf{f})}(z):=\sum_{n=1}^{\infty} \frac{\left(e_{1} n\right) ! \cdots\left(e_{q_{1}} n\right) !}{\left(f_{1} n\right) ! \cdots\left(f_{q_{2}} n\right) !} H_{L n} z^{n} .
$$

On a $q_{L,(\mathbf{e}, \mathbf{f})}(z) \in 1+z \mathbb{Q}[[z]]$ et $z^{-1} q_{(\mathbf{e}, \mathbf{f})}(z)=\left(\prod_{i=1}^{q_{1}} q_{e_{i},(\mathbf{e}, \mathbf{f})}^{e_{i}}(z)\right) /\left(\prod_{j=1}^{q_{2}} q_{f_{j},(\mathbf{e}, \mathbf{f})}^{f_{j}}(z)\right)$, de sorte que si, pour tout $L \in\left\{1, \ldots, M_{(\mathbf{e}, \mathbf{f})}\right\}$, on a $q_{L,(\mathbf{e}, \mathbf{f})} \in \mathbb{Z}[[z]]$, alors on a $q_{(\mathbf{e}, \mathbf{f})} \in z \mathbb{Z}[[z]]$. Ainsi, le point $(i)$ du théorème 2 implique le point $(i)$ du théorème 1 .

Théorème 2. Soit $\boldsymbol{e}$ et $\boldsymbol{f}$ deux suites d'entiers strictement positifs disjointes, vérifiant $|\boldsymbol{e}|=|\boldsymbol{f}|$ et telles que $\mathcal{Q}_{(\boldsymbol{e}, \boldsymbol{f})}$ soit une suite à termes entiers (ce qui équivaut à $\Delta_{(\boldsymbol{e}, \boldsymbol{f})} \geq 0$ sur $[0,1]$ ). On a alors la dichotomie suivante.

(i) Si, pour tout $x \in\left[1 / M_{(\boldsymbol{e}, \boldsymbol{f})}, 1\left[\right.\right.$, on a $\Delta_{(\boldsymbol{e}, \boldsymbol{f})}(x) \geq 1$, alors, pour tout $L \in\left\{1, \ldots, M_{(\boldsymbol{e}, \boldsymbol{f})}\right\}$, on a $q_{L,(\boldsymbol{e}, \boldsymbol{f})}(z) \in \mathbb{Z}[[z]]$.

\footnotetext{
${ }^{3}$ Le cas traité par Lian et Yau correspond au choix des paramètres $k=1$ et $N_{1}=p$ avec $p$ premier.

${ }^{4}$ Voir la fin de la partie 6.2 pour une explication détaillée.
} 
(ii) S'il existe un $x \in\left[1 / M_{(\boldsymbol{e}, \boldsymbol{f})}, 1\left[\right.\right.$ tel que $\Delta_{(\boldsymbol{e}, \boldsymbol{f})}(x)=0$, alors, pour tout $L \in\left\{1, \ldots, M_{(\boldsymbol{e}, \boldsymbol{f})}\right\}$, il n'existe qu'un nombre fini de nombres premiers $p$ tels que $q_{L,(\boldsymbol{e}, \boldsymbol{f})}(z) \in \mathbb{Z}_{p}[[z]]$.

Remarques. - On remarquera l'analogie entre le critère de Landau et les théorèmes 1 et 2.

- Montrer le cas $(i)$ du théorème 1 revient à montrer que la conclusion du théorème bis perdure lorsque $\Delta_{(\mathbf{e}, \mathbf{f})}$ n'est pas forcément croissante sur [0,1[ mais lorsque l'on a la condition plus faible $\Delta_{(\mathbf{e}, \mathbf{f})} \geq 1$ sur $\left[1 / M_{(\mathbf{e}, \mathbf{f})}, 1\left[\right.\right.$. Un exemple d'une telle fonction $\Delta_{(\mathbf{e}, \mathbf{f})}$ est donné par les suites $\mathbf{e}=(3,3)$ et $\mathbf{f}=(2,1,1,1,1)$.

- Le théorème 2 est une généralisation du théorème 1 de 5$]$.

- Si la suite $\mathcal{Q}_{(\mathbf{e}, \mathbf{f})}$ est à termes entiers alors, d'après le critère de Landau, $\Delta_{(\mathbf{e}, \mathbf{f})}$ est positive sur $[0,1]$. Ainsi, s'il existe un $x \in\left[1 / M_{(\mathbf{e}, \mathbf{f})}, 1\left[\right.\right.$ tel que $\Delta_{(\mathbf{e}, \mathbf{f})}(x)<1$, alors on a $\Delta_{(\mathbf{e}, \mathbf{f})}(x)=0$. Ce qui justifie les dichotomies annoncées dans les théorèmes 1 et 2 ,

- Nous verrons en fin de partie 6.2 que $M_{(\mathbf{e}, \mathbf{f})}$ est en fait le terme maximal de la suite e et on a $M_{(\mathbf{e}, \mathbf{f})} \geq 2$.

1.3. Trame de la démonstration des théorèmes 1 et 2. Dans la partie 2, on énonce et démontre notre théorème 3, qui généralise un critère de congruences formelles de Dwork. Ce dernier était crucial pour les résultats de Lian-Yau, Zudilin et Krattenthaler-Rivoal. Le théorème 3 est au coeur de la preuve des théorèmes 1 et 2 puisque l'on montre dans la partie 4.1 que le critère de Dwork ne suffit pas pour démontrer le point $(i)$ du théorème 2 .

Dans la partie 3, on ramène les théorèmes 1 et 2 à la preuve d'un énoncé $p$-adique.

La partie 4 est consacrée à la preuve de l'assertion $(i)$ du théorème 2, ce qui est de loin la partie la plus longue et la plus technique de l'article. On doit en particulier démontrer un certain nombre d'estimations $p$-adiques fines afin d'être en position d'appliquer le théorème 3 .

Dans la partie 5, on démontre les assertions (ii) des théorèmes 1 et 2 qui découlent assez vite de la reformulation de ces théorèmes établie dans la partie 3 .

On finit, dans la partie 6, par caractériser les fonctions hypergéométriques généralisées dont les coefficients peuvent se mettre sous forme de factorielles et on décrit les sauts des applications de Landau sur $[0,1]$.

\section{Congruences Formelles}

La preuve de l'assertion $(i)$ du théorème 2 est basée essentiellement sur la généralisation suivante du théorème de Dwork [3, Theorem 1, p. 296]. Soit $p$ un nombre premier. On note $\Omega$ le complété de la clôture algébrique de $\mathbb{Q}_{p}$ et $\mathcal{O}$ l'anneau des entiers de $\Omega$.

Théorème 3. Fixons un premier $p$. Soit $\left(\boldsymbol{A}_{r}\right)_{r \geq 0}$ une suite d'applications de $\mathbb{N}$ dans $\Omega \backslash\{0\}$ et $\left(\boldsymbol{g}_{r}\right)_{r \geq 0}$ une suite d'applications de $\mathbb{N}$ dans $\mathcal{O} \backslash\{0\}$ telles que, pour tout $r \geq 0$, on ait

(i) $\left|\boldsymbol{A}_{r}(0)\right|_{p}=1$;

(ii) pour tout $m \in \mathbb{N}$, on a $\boldsymbol{A}_{r}(m) \in \boldsymbol{g}_{r}(m) \mathcal{O}$;

et telles qu'il existe un $k_{0} \in \mathbb{N}$ tel que

(iii) pour tout $m \in \mathbb{N}$ et tout $r \geq 0$, si $v_{p}(m) \geq k_{0}$ alors, pour tout $v$, $u$ et $s$ dans $\mathbb{N}$ tels que $v<p, u<p^{s}$, on a

$$
\begin{gathered}
\frac{\boldsymbol{A}_{r}\left(v+u p+m p^{s+1}\right)}{\boldsymbol{A}_{r}(v+u p)}-\frac{\boldsymbol{A}_{r+1}\left(u+m p^{s}\right)}{\boldsymbol{A}_{r+1}(u)} \in p^{s+k_{0}+1} \frac{\boldsymbol{g}_{r+s+1}(m)}{\boldsymbol{g}_{r}(v+u p)} \mathcal{O} ; \\
\text { si } v_{p}(m) \leq k_{0}-1 \text {, alors } \frac{\boldsymbol{A}_{r}(m p)}{\boldsymbol{A}_{r}(0)}-\frac{\boldsymbol{A}_{r+1}(m)}{\boldsymbol{A}_{r+1}(0)} \in p^{v_{p}(m)+1} \boldsymbol{g}_{r+1}(m) \mathcal{O}
\end{gathered}
$$

(iv) pour tout $k \in\left\{1, \ldots, k_{0}\right\}$, tout $v \in\{1, \ldots, p-1\}$, tout $m \in \mathbb{N}$ et tout $r \geq 0$, on a $\boldsymbol{g}_{r}\left(v+m p^{k}\right) \in p^{k} \boldsymbol{g}_{r}\left(m p^{k}\right) \mathcal{O}$ et $\boldsymbol{g}_{r}\left(m p^{k}\right) \in \boldsymbol{g}_{r+k}(m) \mathcal{O}$. 
Alors, pour tout $a \in\{0, \ldots, p-1\}$, tout $m$ et $s$ dans $\mathbb{N}$, tout $r \geq 0$ et tout $K \in \mathbb{Z}$, on a

$$
\begin{aligned}
& \boldsymbol{S}_{r}(a, K, s, p, m):= \\
& \sum_{j=m p^{s}}^{(m+1) p^{s}-1}\left(\boldsymbol{A}_{r}(a+p(K-j)) \boldsymbol{A}_{r+1}(j)-\boldsymbol{A}_{r+1}(K-j) \boldsymbol{A}_{r}(a+j p)\right) \in p^{s+1} \boldsymbol{g}_{s+r+1}(m) \mathcal{O},
\end{aligned}
$$

où l'on pose, pour tout $r \geq 0, \boldsymbol{A}_{r}(\ell)=0$ si $\ell<0$.

Remarques. - Le théorème de Dwork correspond au cas où $k_{0}=0$, auquel cas la condition (iii) est identique à la condition (iii) du critère de Dwork et la condition (iv) est vide. La preuve pour $k_{0} \geq 1$ est très différente de celle donnée par Dwork pour $k_{0}=0$.

- On peut trouver une généralisation différente du critère de Dwork dans [10]. Cette généralisation ne semble pas adaptée à notre situation.

- Les fonctions $\mathbf{A}_{r}$ et $\mathbf{g}_{r}$ peuvent dépendre de $p$. On utilisera cette souplesse.

Dans la suite, si $k_{0}$ est un entier naturel, on appellera $k_{0}$-couple de Dwork tout couple de suites $\left(\left(\mathbf{A}_{r}\right)_{r>0},\left(\mathbf{g}_{r}\right)_{r>0}\right)$ où les $\mathbf{A}_{r}$ sont des applications de $\mathbb{N}$ dans $\Omega \backslash\{0\}$, les $\mathbf{g}_{r}$ sont des applications de $\mathbb{N}$ dans $\mathcal{O} \backslash\{0\}$, telles que, pour tout $r \geq 0, \mathbf{A}_{r}$ et $\mathbf{g}_{r}$ vérifient les conditions $(i),(i i),(i i i)$ et $(i v)$ du théorème 3 .

Le but de la fin de cette partie est de démontrer le théorème [3. Pour cela, on va avoir besoin d'un certain nombre de résultats intermédiaires.

\subsection{Lemmes préparatoires. On énonce et démontre trois lemmes.}

Lemme 1. Soit $\left(\boldsymbol{g}_{r}\right)_{r \geq 0}$ une suite d'applications de $\mathbb{N}$ dans $\mathcal{O} \backslash\{0\}$ telle qu'il existe un entier naturel $k_{0} \geq 1$ tel que

$(I V)$ pour tout $k \in\left\{1, \ldots, k_{0}\right\}$, tout $v \in\{1, \ldots, p-1\}$, tout $m \in \mathbb{N}$ et tout $r \geq 0$, on a $\boldsymbol{g}_{r}\left(v+m p^{k}\right) \in p^{k} \boldsymbol{g}_{r}\left(m p^{k}\right) \mathcal{O}$ et $\boldsymbol{g}_{r}\left(m p^{k}\right) \in \boldsymbol{g}_{r+k}(m) \mathcal{O}$.

Alors, pour tout $w \in \mathbb{N}, w \geq 1$, et tout $r \geq 0$, on a $\boldsymbol{g}_{r}(w) \in p^{k_{0}} \mathcal{O}$.

Remarque. - La condition $(I V)$ du lemme 1 est la condition $(i v)$ du théorème 3 .

- La condition $w \neq 0$ est essentielle car, pour $w=0$, l'hypothèse $(I V)$ ne donne pas mieux que $\mathbf{g}_{r}(0) \in \mathcal{O} \backslash\{0\}$.

Démonstration. On écrit $w:=\sum_{\ell=0}^{N} w_{\ell} p^{\ell}$ le développement $p$-adique de $w$, où $w_{N} \neq 0$. On va raisonner par récurrence sur $N$.

- Supposons $N=0$.

Dans ce cas, on a $w=w_{0} \in\{1, \ldots, p-1\}$. En appliquant $(I V)$ avec $v=w, k=k_{0}$ et $m=0$, on obtient bien $\mathbf{g}_{r}(w) \in p^{k_{0}} \mathbf{g}_{r}(0) \mathcal{O} \subset p^{k_{0}} \mathcal{O}$.

- Supposons $N \geq 1$.

Soit $\eta$ le plus petit entier naturel tel que $w_{\eta} \neq 0$. Si $\eta=0$, alors $w_{0} \geq 1$ donc, d'après $(I V)$ appliquée en $v=w_{0}, k=1$ et $m=\sum_{\ell=0}^{N-1} w_{\ell+1} p^{\ell}$, on obtient $\mathbf{g}_{r}(w) \in p \mathbf{g}_{r}(m p) \mathcal{O} \subset p \mathbf{g}_{r+1}(m) \mathcal{O}$. On a $m \geq w_{N} p^{N-1}>0$. Ainsi, par hypothèse de récurrence, on a $\mathbf{g}_{r+1}(m) \in p^{k_{0}} \mathcal{O}$, d'où le résultat.

Si $\eta \geq 1$, alors $w=p \sum_{\ell=0}^{N-1} w_{\ell+1} p^{\ell}$. D'après $(I V)$ appliquée en $k=1$ et $m=\sum_{\ell=0}^{N-1} w_{\ell+1} p^{\ell}>0$, on obtient $\mathbf{g}_{r}(w)=\mathbf{g}_{r}(m p) \in \mathbf{g}_{r+1}(m) \mathcal{O}$ et on conclut par l'hypothèse de récurrence. Ceci achève la preuve du lemme.

Lemme 2. Soit $\left(\left(\boldsymbol{A}_{r}\right)_{r \geq 0},\left(\boldsymbol{g}_{r}\right)_{r \geq 0}\right)$ un $k_{0}$-couple de Dwork avec $k_{0} \geq 1$. Alors, pour tout $k \in$ $\left\{0, \ldots, k_{0}\right\}$, tout $a \in\{0, \ldots, p-1\}$, tout $m \in \mathbb{N}$, tout $K \in \mathbb{Z}$ et tout $r \geq 0$, on a $\boldsymbol{S}_{r}\left(a, K, 0, p, m p^{k}\right) \in$ $p^{k+1} \boldsymbol{g}_{r+1}\left(m p^{k}\right) \mathcal{O}$. 
Démonstration. Soit $k \in\left\{0, \ldots, k_{0}\right\}, a \in\{0, \ldots, p-1\}, m \in \mathbb{N}, K \in \mathbb{Z}$ et $r \geq 0$. On a

$$
\mathbf{S}_{r}\left(a, K, 0, p, m p^{k}\right)=\mathbf{A}_{r}\left(a+p\left(K-m p^{k}\right)\right) \mathbf{A}_{r+1}\left(m p^{k}\right)-\mathbf{A}_{r+1}\left(K-m p^{k}\right) \mathbf{A}_{r}\left(a+m p^{k+1}\right) .
$$

Si $K-m p^{k}<0$ alors $\mathbf{S}_{r}\left(a, K, 0, p, m p^{k}\right)=0$ et le lemme 2 est trivialement vrai. On suppose donc $K-m p^{k} \geq 0$ dans la suite de la démonstration. On va distinguer plusieurs cas.

- Supposons $a \neq 0$ et $k \leq k_{0}-1$.

D'après $(i i)$, on a $\mathbf{A}_{r}\left(a+p\left(K-m p^{k}\right)\right) \in \mathbf{g}_{r}\left(a+p\left(K-m p^{k}\right)\right) \mathcal{O}$ et, d'après le lemme 11, on a $\mathbf{g}_{r}\left(a+p\left(K-m p^{k}\right)\right) \in p^{k_{0}} \mathcal{O}$ car $a+p\left(K-m p^{k}\right) \geq 1$. Comme $k+1 \leq k_{0}$, on obtient donc

$$
\mathbf{A}_{r}\left(a+p\left(K-m p^{k}\right)\right) \in p^{k+1} \mathcal{O} .
$$

Toujours d'après $(i i)$, on a

$$
\begin{gathered}
\mathbf{A}_{r+1}\left(m p^{k}\right) \in \mathbf{g}_{r+1}\left(m p^{k}\right) \mathcal{O}, \\
\mathbf{A}_{r+1}\left(K-m p^{k}\right) \in \mathbf{g}_{r+1}\left(K-m p^{k}\right) \mathcal{O} \subset \mathcal{O}
\end{gathered}
$$

et $\mathbf{A}_{r}\left(a+m p^{k+1}\right) \in \mathbf{g}_{r}\left(a+m p^{k+1}\right) \mathcal{O}$. Comme $1 \leq k+1 \leq k_{0}$, on peut appliquer $(i v)$ en $k+1$ et on obtient $\mathbf{g}_{r}\left(a+m p^{k+1}\right) \in p^{k+1} \mathbf{g}_{r}\left(m p^{k+1}\right) \mathcal{O} \subset p^{k+1} \mathbf{g}_{r+1}\left(m p^{k}\right) \mathcal{O}$ et donc

$$
\mathbf{A}_{r}\left(a+m p^{k+1}\right) \in p^{k+1} \mathbf{g}_{r+1}\left(m p^{k}\right) \mathcal{O} .
$$

Ainsi, d'après (2.1), (2.2), (2.3) et (2.4), on obtient bien $\mathbf{S}_{r}\left(a, K, 0, p, m p^{k}\right) \in p^{k+1} \mathbf{g}_{r+1}\left(m p^{k}\right) \mathcal{O}$.

- Supposons $a \neq 0, k=k_{0}$ et $K-m p^{k_{0}}>0$.

D'après $(i i)$, on a $\mathbf{A}_{r}\left(a+p\left(K-m p^{k_{0}}\right)\right) \in \mathbf{g}_{r}\left(a+p\left(K-m p^{k_{0}}\right)\right) \mathcal{O}$ et, d'après $(i v)$, on a $\mathbf{g}_{r}\left(a+p\left(K-m p^{k_{0}}\right)\right) \in p \mathbf{g}_{r}\left(p\left(K-m p^{k_{0}}\right)\right) \mathcal{O}$. Comme $K-m p^{k_{0}}>0$, le lemme 1 implique donc que

$$
\mathbf{A}_{r}\left(a+p\left(K-m p^{k_{0}}\right)\right) \in p^{k_{0}+1} \mathcal{O} \text {. }
$$

D'après $(i i)$, on a

$$
\mathbf{A}_{r+1}\left(m p^{k_{0}}\right) \in \mathbf{g}_{r+1}\left(m p^{k_{0}}\right) \mathcal{O}
$$

et

$$
\mathbf{A}_{r+1}\left(K-m p^{k_{0}}\right) \in \mathbf{g}_{r+1}\left(K-m p^{k_{0}}\right) \mathcal{O} \subset p^{k_{0}} \mathcal{O},
$$

où l'inclusion dans (2.7) est obtenue de nouveau via le lemme1.

Enfin, d'après $(i i)$, on a $\mathbf{A}_{r}\left(a+m p^{k_{0}+1}\right) \in \mathbf{g}_{r}\left(a+m p^{k_{0}+1}\right) \mathcal{O}$. D'après $(i v)$, on obtient

$$
\mathbf{g}_{r}\left(a+m p^{k_{0}+1}\right)=\mathbf{g}_{r}\left(a+\left(m p^{k_{0}}\right) p\right) \in p \mathbf{g}_{r}\left(m p^{k_{0}+1}\right) \mathcal{O} \subset p \mathbf{g}_{r+1}\left(m p^{k_{0}}\right) \mathcal{O}
$$

et donc

$$
\mathbf{A}_{r}\left(a+m p^{k_{0}+1}\right) \in p \mathbf{g}_{r+1}\left(m p^{k_{0}}\right) \mathcal{O} .
$$

Ainsi, d'après (2.5), (2.6) , (2.7) et (2.8) , on obtient bien $\mathbf{S}_{r}\left(a, K, 0, p, m p^{k_{0}}\right) \in p^{k_{0}+1} \mathbf{g}_{r+1}\left(m p^{k_{0}}\right) \mathcal{O}$.

- Supposons $a \neq 0, k=k_{0}$ et $K-m p^{k_{0}}=0$. On a alors

$$
\begin{aligned}
\mathbf{S}_{r}\left(a, K, 0, p, m p^{k_{0}}\right) & =\mathbf{A}_{r}(a) \mathbf{A}_{r+1}\left(m p^{k_{0}}\right)-\mathbf{A}_{r+1}(0) \mathbf{A}_{r}\left(a+m p^{k_{0}+1}\right) \\
& =-\mathbf{A}_{r}(a) \mathbf{A}_{r+1}(0)\left(\frac{\mathbf{A}_{r}\left(a+m p^{k_{0}+1}\right)}{\mathbf{A}_{r}(a)}-\frac{\mathbf{A}_{r+1}\left(m p^{k_{0}}\right)}{\mathbf{A}_{r+1}(0)}\right),
\end{aligned}
$$

avec, d'après $(i)$ et $(i i), \mathbf{A}_{r}(a) \mathbf{A}_{r+1}(0) \in \mathbf{g}_{r}(a) \mathcal{O}$. Comme $v_{p}\left(m p^{k_{0}}\right) \geq k_{0}$, on obtient, d'après $(i i i)$ appliquée en $s=0, v=a, u=0$ et $m p^{k_{0}}$ à la place de $m$, que

$$
\frac{\mathbf{A}_{r}\left(a+m p^{k_{0}+1}\right)}{\mathbf{A}_{r}(a)}-\frac{\mathbf{A}_{r+1}\left(m p^{k_{0}}\right)}{\mathbf{A}_{r+1}(0)} \in p^{k_{0}+1} \frac{\mathbf{g}_{r+1}\left(m p^{k_{0}}\right)}{\mathbf{g}_{r}(a)} \mathcal{O} .
$$

La congruence voulue en découle.

- Il reste un seul cas : $a=0$. 
Dans ce cas, on a

$$
\begin{aligned}
& \mathbf{S}_{r}\left(0, K, 0, p, m p^{k}\right)=\mathbf{A}_{r}\left(p\left(K-m p^{k}\right)\right) \mathbf{A}_{r+1}\left(m p^{k}\right)-\mathbf{A}_{r+1}\left(K-m p^{k}\right) \mathbf{A}_{r}\left(m p^{k+1}\right) \\
& =\mathbf{A}_{r}(0) \mathbf{A}_{r+1}(0)\left(\frac{\mathbf{A}_{r}\left(p\left(K-m p^{k}\right)\right)}{\mathbf{A}_{r}(0)} \frac{\mathbf{A}_{r+1}\left(m p^{k}\right)}{\mathbf{A}_{r+1}(0)}-\frac{\mathbf{A}_{r+1}\left(K-m p^{k}\right)}{\mathbf{A}_{r+1}(0)} \frac{\mathbf{A}_{r}\left(m p^{k+1}\right)}{\mathbf{A}_{r}(0)}\right) .
\end{aligned}
$$

On écrit le terme de droite sous la forme

$$
\begin{aligned}
& \frac{\mathbf{A}_{r}\left(p\left(K-m p^{k}\right)\right)}{\mathbf{A}_{r}(0)} \frac{\mathbf{A}_{r+1}\left(m p^{k}\right)}{\mathbf{A}_{r+1}(0)}-\frac{\mathbf{A}_{r+1}\left(K-m p^{k}\right)}{\mathbf{A}_{r+1}(0)} \frac{\mathbf{A}_{r}\left(m p^{k+1}\right)}{\mathbf{A}_{r}(0)}= \\
& \frac{\mathbf{A}_{r+1}\left(m p^{k}\right)}{\mathbf{A}_{r+1}(0)}\left(\frac{\mathbf{A}_{r}\left(p\left(K-m p^{k}\right)\right)}{\mathbf{A}_{r}(0)}-\frac{\mathbf{A}_{r+1}\left(K-m p^{k}\right)}{\mathbf{A}_{r+1}(0)}\right) \\
&-\frac{\mathbf{A}_{r+1}\left(K-m p^{k}\right)}{\mathbf{A}_{r+1}(0)}\left(\frac{\mathbf{A}_{r}\left(m p^{k+1}\right)}{\mathbf{A}_{r}(0)}-\frac{\mathbf{A}_{r+1}\left(m p^{k}\right)}{\mathbf{A}_{r+1}(0)}\right) .
\end{aligned}
$$

Remarquons maintenant que l'on a

$$
\frac{\mathbf{A}_{r}\left(m p^{k+1}\right)}{\mathbf{A}_{r}(0)}-\frac{\mathbf{A}_{r+1}\left(m p^{k}\right)}{\mathbf{A}_{r+1}(0)} \in p^{k+1} \mathbf{g}_{r+1}\left(m p^{k}\right) \mathcal{O}
$$

En effet, si $v_{p}\left(m p^{k}\right) \geq k_{0}$ alors, en appliquant $(i i i)$ avec $v=u=s=0$ et $m p^{k}$ à la place de $m$, on obtient

$$
\frac{\mathbf{A}_{r}\left(m p^{k+1}\right)}{\mathbf{A}_{r}(0)}-\frac{\mathbf{A}_{r+1}\left(m p^{k}\right)}{\mathbf{A}_{r+1}(0)} \in p^{k_{0}+1} \frac{\mathbf{g}_{r+1}\left(m p^{k}\right)}{\mathbf{g}_{r}(0)} \mathcal{O} .
$$

De plus, d'après $(i)$ et $(i i), \mathbf{g}_{r}(0)$ est inversible dans $\mathcal{O}$ et, par hypothèse, on a $k \leq k_{0}$, donc on obtient bien (2.9) dans ce cas. Si en revanche $v_{p}\left(m p^{k}\right) \leq k_{0}-1$ alors, d'après $(i i i)$ avec $m p^{k}$ à la place de $m$, on obtient

$$
\frac{\mathbf{A}_{r}\left(m p^{k+1}\right)}{\mathbf{A}_{r}(0)}-\frac{\mathbf{A}_{r+1}\left(m p^{k}\right)}{\mathbf{A}_{r+1}(0)} \in p^{v_{p}\left(m p^{k}\right)+1} \mathbf{g}_{r+1}\left(m p^{k}\right) \mathcal{O} \subset p^{k+1} \mathbf{g}_{r+1}\left(m p^{k}\right) \mathcal{O},
$$

ce qui achève la vérification de (2.9).

Ainsi, si $K-m p^{k}=0$, alors on a bien $\mathbf{S}_{r}\left(0, K, 0, p, m p^{k}\right) \in p^{k+1} \mathbf{g}_{r+1}\left(m p^{k}\right) \mathcal{O}$. Si $K-m p^{k}>0$, alors on a

$$
\frac{\mathbf{A}_{r}\left(p\left(K-m p^{k}\right)\right)}{\mathbf{A}_{r}(0)}-\frac{\mathbf{A}_{r+1}\left(K-m p^{k}\right)}{\mathbf{A}_{r+1}(0)} \in p \mathbf{g}_{r+1}\left(K-m p^{k}\right) \mathcal{O} .
$$

En effet, si $v_{p}\left(K-m p^{k}\right) \geq k_{0}$ alors, en appliquant $(i i i)$ avec $v=u=s=0$ et $K-m p^{k}$ à la place de $m$, on obtient

$$
\frac{\mathbf{A}_{r}\left(p\left(K-m p^{k}\right)\right)}{\mathbf{A}_{r}(0)}-\frac{\mathbf{A}_{r+1}\left(K-m p^{k}\right)}{\mathbf{A}_{r+1}(0)} \in p^{k_{0}+1} \frac{\mathbf{g}_{r+1}\left(K-m p^{k}\right)}{\mathbf{g}_{r}(0)} \mathcal{O} .
$$

De plus, d'après $(i)$ et $(i i), \mathbf{g}_{r}(0)$ est inversible dans $\mathcal{O}$ et, par hypothèse, on a $k_{0} \geq 1$, donc on obtient bien (2.10) dans ce cas. Si en revanche $v_{p}\left(K-m p^{k}\right) \leq k_{0}-1$ alors, d'après (iii) avec $K-m p^{k}$ à la place de $m$, on obtient

$$
\frac{\mathbf{A}_{r}\left(p\left(K-m p^{k}\right)\right)}{\mathbf{A}_{r}(0)}-\frac{\mathbf{A}_{r+1}\left(K-m p^{k}\right)}{\mathbf{A}_{r+1}(0)} \in p^{v_{p}\left(K-m p^{k}\right)+1} \mathbf{g}_{r+1}\left(K-m p^{k}\right) \mathcal{O} \subset p \mathbf{g}_{r+1}\left(K-m p^{k}\right) \mathcal{O},
$$

ce qui achève la vérification de (2.10). 
D'après le lemme 1, on a $\mathbf{g}_{r+1}\left(K-m p^{k}\right) \in p^{k_{0}} \mathcal{O}$. D'après $(i)$ et $(i i)$, on a de plus $\frac{\mathbf{A}_{r+1}\left(m p^{k}\right)}{\mathbf{A}_{r+1}(0)} \in$ $\mathbf{g}_{r+1}\left(m p^{k}\right) \mathcal{O}$, donc

$$
\mathbf{A}_{r}(0) \mathbf{A}_{r+1}(0) \frac{\mathbf{A}_{r+1}\left(m p^{k}\right)}{\mathbf{A}_{r+1}(0)}\left(\frac{\mathbf{A}_{r}\left(p\left(K-m p^{k}\right)\right)}{\mathbf{A}_{r}(0)}-\frac{\mathbf{A}_{r+1}\left(K-m p^{k}\right)}{\mathbf{A}_{r+1}(0)}\right) \in p^{k_{0}+1} \mathbf{g}_{r+1}\left(m p^{k}\right) \mathcal{O}
$$

et comme également $\frac{\mathbf{A}_{r+1}\left(K-m p^{k}\right)}{\mathbf{A}_{r+1}(0)} \in \mathbf{g}_{r+1}\left(K-m p^{k}\right) \mathcal{O} \subset \mathcal{O}$, on a

$$
\mathbf{A}_{r}(0) \mathbf{A}_{r+1}(0) \frac{\mathbf{A}_{r+1}\left(K-m p^{k}\right)}{\mathbf{A}_{r+1}(0)} \cdot\left(\frac{\mathbf{A}_{r}\left(m p^{k+1}\right)}{\mathbf{A}_{r}(0)}-\frac{\mathbf{A}_{r+1}\left(m p^{k}\right)}{\mathbf{A}_{r+1}(0)}\right) \in p^{k+1} \mathbf{g}_{r+1}\left(m p^{k}\right) \mathcal{O} .
$$

On a donc bien $\mathbf{S}_{r}\left(0, K, 0, p, m p^{k}\right) \in p^{k+1} \mathbf{g}_{r+1}\left(m p^{k}\right) \mathcal{O}$, ce qui achève la preuve du lemme.

Enfin, on aura besoin du lemme suivant.

Lemme 3. Soit $\left(\left(\boldsymbol{A}_{r}\right)_{r \geq 0},\left(\boldsymbol{g}_{r}\right)_{r \geq 0}\right)$ un $k_{0}$-couple de Dwork avec $k_{0} \geq 1$. Soit $a \in\{0, \ldots, p-1\}$, $K \in \mathbb{Z}$ et $s \in \mathbb{N}, s \geq 1$. Si, pour tout $s_{0}<s$, pour tout $m \in \mathbb{N}$ et tout $r \geq 0$, on a

$$
\boldsymbol{S}_{r}\left(a, K, s_{0}, p, m p^{k_{0}}\right) \in p^{s_{0}+k_{0}+1} \boldsymbol{g}_{s_{0}+r+1}\left(m p^{k_{0}}\right) \mathcal{O},
$$

et

$$
\boldsymbol{S}_{r}\left(a, K, s_{0}, p, m\right) \in p^{s_{0}+1} \boldsymbol{g}_{s_{0}+r+1}(m) \mathcal{O}
$$

Alors, pour tout $m \in \mathbb{N}$ et tout $r \geq 0$, on a $\boldsymbol{S}_{r}(a, K, s, p, m) \in p^{s+1} \boldsymbol{g}_{s+r+1}(m) \mathcal{O}$.

Démonstration. On va d'abord montrer que, pour tout $m \in \mathbb{N}$, tout $r \geq 1$ et tout $k \leq \min \left(s, k_{0}\right)$, on a $\mathbf{S}_{r}(a, K, s, p, m) \equiv \mathbf{S}_{r}\left(a, K, s-k, p, m p^{k}\right) \bmod p^{s+1} \mathbf{g}_{s+r+1}(m) \mathcal{O}$. Pour cela, on va raisonner par récurrence sur $k$.

Si $k=0$, il n'y a rien à montrer.

Supposons que $\min \left(s, k_{0}\right) \geq k \geq 1$. Par hypothèse de récurrence, on a

$$
\mathbf{S}_{r}(a, K, s, p, m) \equiv \mathbf{S}_{r}\left(a, K, s-k+1, p, m p^{k-1}\right) \quad \bmod p^{s+1} \mathbf{g}_{s+r+1}(m) \mathcal{O} .
$$

Comme $\left\{m p^{s}, \ldots, m p^{s}+p^{s-k+1}-1\right\}=\bigcup_{v=0}^{p-1}\left\{m p^{s}+v p^{s-k}, \ldots, m p^{s}+v p^{s-k}+p^{s-k}-1\right\}$, on a

$$
\mathbf{S}_{r}\left(a, K, s-k+1, p, m p^{k-1}\right)=\sum_{v=0}^{p-1} \mathbf{S}_{r}\left(a, K, s-k, p, v+m p^{k}\right) .
$$

Comme $s \geq k \geq 1$, on a $0 \leq s-k<s$ et on obtient, d'après (2.12), que, pour tout $v \in\{0, \ldots, p-1\}$, on a $\mathbf{S}_{r}\left(a, K, s-k, p, v+m p^{k}\right) \in p^{s-k+1} \mathbf{g}_{s-k+r+1}\left(v+m p^{k}\right) \mathcal{O}$. Comme également $k \in\left\{1, \ldots, k_{0}\right\}$ on obtient d'après $(i v)$, que, pour tout $v \in\{1, \ldots, p-1\}$, on a

$$
\mathbf{g}_{s-k+r+1}\left(v+m p^{k}\right) \in p^{k} \mathbf{g}_{s-k+r+1}\left(m p^{k}\right) \mathcal{O} \subset p^{k} \mathbf{g}_{s+r+1}(m) \mathcal{O} .
$$

En utilisant ces informations dans (2.14), on obtient (il reste seulement le terme pour $v=0$ ) :

$$
\mathbf{S}_{r}\left(a, K, s-k+1, p, m p^{k-1}\right) \equiv \mathbf{S}_{r}\left(a, K, s-k, p, m p^{k}\right) \quad \bmod p^{s+1} \mathbf{g}_{s+r+1}(m) \mathcal{O},
$$

ce qui, joint à (2.13), montre que $\mathbf{S}_{r}(a, K, s, p, m) \equiv \mathbf{S}_{r}\left(a, K, s-k, p, m p^{k}\right) \bmod p^{s+1} \mathbf{g}_{s+r+1}(m) \mathcal{O}$ et achève la récurrence sur $k$.

On a donc $\mathbf{S}_{r}(a, K, s, p, m) \equiv \mathbf{S}_{r}\left(a, K, s-\min \left(s, k_{0}\right), p, m p^{\min \left(s, k_{0}\right)}\right) \bmod p^{s+1} \mathbf{g}_{s+r+1}(m) \mathcal{O}$.

Si $s<k_{0}$, alors $\mathbf{S}_{r}(a, K, s, p, m) \equiv \mathbf{S}_{r}\left(a, K, 0, p, m p^{s}\right) \bmod p^{s+1} \mathbf{g}_{s+r+1}(m) \mathcal{O}$. En appliquant le lemme 2 en $k=s$, puis $(i v)$ en $k=s$, on obtient $\mathbf{S}_{r}\left(a, K, 0, p, m p^{s}\right) \in p^{s+1} \mathbf{g}_{r+1}\left(m p^{s}\right) \mathcal{O} \subset$ $p^{s+1} \mathbf{g}_{s+r+1}(m) \mathcal{O}$ et donc $\mathbf{S}_{r}(a, K, s, p, m) \in p^{s+1} \mathbf{g}_{s+r+1}(m) \mathcal{O}$, comme voulu.

Si maintenant $s \geq k_{0}$ alors $\mathbf{S}_{r}(a, K, s, p, m) \equiv \mathbf{S}_{r}\left(a, K, s-k_{0}, p, m p^{k_{0}}\right) \bmod p^{s+1} \mathbf{g}_{s+r+1}(m) \mathcal{O}$. Or, comme $s \geq k_{0} \geq 1$, on a $0 \leq s-k_{0}<s$ et on obtient (d'après (2.11) et (iv) appliquée en 
$\left.k=k_{0}\right)$ que $\mathbf{S}_{r}\left(a, K, s-k_{0}, p, m p^{k_{0}}\right) \in p^{s-k_{0}+k_{0}+1} \mathbf{g}_{s-k_{0}+r+1}\left(m p^{k_{0}}\right) \mathcal{O} \subset p^{s+1} \mathbf{g}_{s+r+1}(m) \mathcal{O}$, ce qui achève la preuve du lemme.

2.2. Démonstration du théorème 3. Comme dit au début de la partie 2, le cas $k_{0}=0$ correspond au critère de Dwork. Il nous suffit donc de démontrer le cas où il existe un $k_{0} \geq 1$ tel que les hypothèses $(i i i)$ et $(i v)$ soient vérifiées. En particulier, on utilisera les lemmes 1, 2 et 3, La trame de la démonstration s'inspire de celle du théorème de Dwork, mais elle diffère assez sensiblement dans les détails.

On doit montrer que, pour tout $a \in\{0, \ldots, p-1\}$, tout $m$ et $s$ dans $\mathbb{N}$, tout $r \geq 0$ et tout $K \in \mathbb{Z}$, on a

$$
\mathbf{S}_{r}(a, K, s, p, m) \in p^{s+1} \mathbf{g}_{s+r+1}(m) \mathcal{O} .
$$

Pour tout $a \in\{0, \ldots, p-1\}$, tout $j \in \mathbb{N}$, tout $r \geq 0$ et tout $K \in \mathbb{Z}$, on note

$$
\mathbf{U}_{r}(a, K, p, j):=\mathbf{A}_{r}(a+p(K-j)) \mathbf{A}_{r+1}(j)-\mathbf{A}_{r+1}(K-j) \mathbf{A}_{r}(a+j p),
$$

de sorte que $\mathbf{S}_{r}(a, K, s, p, m)=\sum_{j=m p^{s}}^{(m+1) p^{s}-1} \mathbf{U}_{r}(a, K, p, j)$. Pour tout $s \in \mathbb{N}, s \geq 1$, on note $\alpha_{s}$ l'assertion suivante : pour tout $a \in\{0, \ldots, p-1\}$, tout $u \in\{0, \ldots, s-1\}$, tout $m \in \mathbb{N}$, tout $r \geq 0$ et tout $K \in \mathbb{Z}$, on a les congruences

$$
\mathbf{S}_{r}(a, K, u, p, m) \in p^{u+1} \mathbf{g}_{u+r+1}(m) \mathcal{O} \quad \text { et } \quad \mathbf{S}_{r}\left(a, K, u, p, m p^{k_{0}}\right) \in p^{u+k_{0}+1} \mathbf{g}_{u+r+1}\left(m p^{k_{0}}\right) \mathcal{O} .
$$

Pour tout $s \in \mathbb{N}, s \geq 1$, et tout $t \in\{0, \ldots, s\}$, on note $\beta_{t, s}$ l'assertion suivante : pour tout $a \in\{0, \ldots, p-1\}$, tout $m \in \mathbb{N}$, tout $r \geq 0$ et tout $K \in \mathbb{Z}$, on a la congruence

$$
\begin{aligned}
& \mathbf{S}_{r}\left(a, K+m p^{s+k_{0}}, s, p, m p^{k_{0}}\right) \equiv \\
& \sum_{j=0}^{p^{s-t}-1} \frac{\mathbf{A}_{t+r+1}\left(j+m p^{s-t+k_{0}}\right)}{\mathbf{A}_{t+r+1}(j)} \mathbf{S}_{r}(a, K, t, p, j) \quad \bmod p^{s+k_{0}+1} \mathbf{g}_{s+r+1}\left(m p^{k_{0}}\right) \mathcal{O} .
\end{aligned}
$$

Nous allons maintenant énoncer trois lemmes permettant de montrer (2.15).

Lemme 4. L'assertion $\alpha_{1}$ est vraie.

Lemme 5. Pour tout $s, r$ et $m$ dans $\mathbb{N}$, tout $a \in\{0, \ldots, p-1\}$, tout $j \in\left\{0, \ldots, p^{s}-1\right\}$ et tout $K \in \mathbb{Z}$, on $a$

$\boldsymbol{U}_{r}\left(a, K+m p^{s+k_{0}}, p, j+m p^{s+k_{0}}\right) \equiv \frac{\boldsymbol{A}_{r+1}\left(j+m p^{s+k_{0}}\right)}{\boldsymbol{A}_{r+1}(j)} \boldsymbol{U}_{r}(a, K, p, j) \quad \bmod p^{s+k_{0}+1} \boldsymbol{g}_{s+r+1}\left(m p^{k_{0}}\right) \mathcal{O}$.

Lemme 6. Pour tout $s \in \mathbb{N}, s \geq 1$, et tout $t \in\{0, \ldots, s-1\}$, les assertions $\alpha_{s}$ et $\beta_{t, s}$ impliquent l'assertion $\beta_{t+1, s}$.

Avant de prouver ces lemmes, nous allons montrer que leur validité implique bien (2.15). On va montrer que $\alpha_{s}$ est vraie pour tout $s \geq 1$ par récurrence sur $s$, ce qui donnera en particulier la conclusion du théorème [3. D'après le lemme 4, $\alpha_{1}$ est vraie. Supposons $\alpha_{s}$ vraie pour un $s \geq 1$ fixé. On remarque que $\beta_{0, s}$ est l'assertion

$$
\begin{aligned}
\beta_{0, s}: \mathbf{S}_{r}\left(a, K+m p^{s+k_{0}}, s, p, m p^{k_{0}}\right) \equiv \\
\qquad \sum_{j=0}^{p^{s}-1} \frac{\mathbf{A}_{r+1}\left(j+m p^{s+k_{0}}\right)}{\mathbf{A}_{r+1}(j)} \mathbf{S}_{r}(a, K, 0, p, j) \quad \bmod p^{s+k_{0}+1} \mathbf{g}_{s+r+1}\left(m p^{k_{0}}\right) \mathcal{O} .
\end{aligned}
$$


Comme $\mathbf{S}_{r}(a, K, 0, p, j)=\mathbf{U}_{r}(a, K, p, j)$, on a

$$
\sum_{j=0}^{p^{s}-1} \frac{\mathbf{A}_{r+1}\left(j+m p^{s+k_{0}}\right)}{\mathbf{A}_{r+1}(j)} \mathbf{S}_{r}(a, K, 0, p, j)=\sum_{j=0}^{p^{s}-1} \frac{\mathbf{A}_{r+1}\left(j+m p^{s+k_{0}}\right)}{\mathbf{A}_{r+1}(j)} \mathbf{U}_{r}(a, K, p, j)
$$

et, d'après le lemme 5, on obtient

$$
\begin{aligned}
\sum_{j=0}^{p^{s}-1} & \frac{\mathbf{A}_{r+1}\left(j+m p^{s+k_{0}}\right)}{\mathbf{A}_{r+1}(j)} \mathbf{U}_{r}(a, K, p, j) \\
& \equiv \sum_{j=0}^{p^{s}-1} \mathbf{U}_{r}\left(a, K+m p^{s+k_{0}}, p, j+m p^{s+k_{0}}\right) \quad \bmod p^{s+k_{0}+1} \mathbf{g}_{s+r+1}\left(m p^{k_{0}}\right) \mathcal{O} \\
& \equiv \mathbf{S}_{r}\left(a, K+m p^{s+k_{0}}, s, p, m p^{k_{0}}\right) \quad \bmod p^{s+k_{0}+1} \mathbf{g}_{s+r+1}\left(m p^{k_{0}}\right) \mathcal{O}
\end{aligned}
$$

Ainsi, l'assertion $\beta_{0, s}$ est vraie. On obtient alors, par le lemme 6, la validité de $\beta_{1, s}$. Par itération $\mathrm{du}$ lemme 6. on obtient finalement $\beta_{s, s}$, qui est l'assertion

$$
\mathbf{S}_{r}\left(a, K+m p^{s+k_{0}}, s, p, m p^{k_{0}}\right) \equiv \frac{\mathbf{A}_{s+r+1}\left(m p^{k_{0}}\right)}{\mathbf{A}_{s+r+1}(0)} \mathbf{S}_{r}(a, K, s, p, 0) \quad \bmod p^{s+k_{0}+1} \mathbf{g}_{s+r+1}\left(m p^{k_{0}}\right) \mathcal{O}
$$

Nous allons maintenant montrer que, pour tout $a \in\{0, \ldots, p-1\}$, tout $s \in \mathbb{N}$, tout $r \geq 0$ et tout $K \in \mathbb{Z}$, on a $\mathbf{S}_{r}(a, K, s, p, 0) \in p^{s+k_{0}+1} \mathcal{O}$. Soit $T \in \mathbb{N}$ tel que $(T+1) p^{s}>K$. On a

$$
\begin{aligned}
\sum_{m=0}^{T} \mathbf{S}_{r}(a, K, s, p, m) & =\sum_{m=0}^{T} \sum_{j=m p^{s}}^{(m+1) p^{s}-1}\left(\mathbf{A}_{r}(a+p(K-j)) \mathbf{A}_{r+1}(j)-\mathbf{A}_{r+1}(K-j) \mathbf{A}_{r}(a+j p)\right) \\
& =\sum_{j=0}^{K}\left(\mathbf{A}_{r}(a+p(K-j)) \mathbf{A}_{r+1}(j)-\mathbf{A}_{r+1}(K-j) \mathbf{A}_{r}(a+j p)\right) \\
& =0
\end{aligned}
$$

où l'on a utilisé dans (2.17) le fait que $\mathbf{A}_{r}(\ell)=0$ pour $\ell<0$, et (2.18) a lieu car le terme de la somme (2.17) est changé en son opposé lorsque l'on change l'indice $j$ en $K-j$.

Comme $\alpha_{s}$ est vraie, on obtient via le lemme 3 (avec $\left.s_{0}=u\right): \mathbf{S}_{r}(a, K, s, p, m) \in p^{s+1} \mathbf{g}_{s+r+1}(m) \mathcal{O}$, ce qui prouve la première partie de l'assertion $\alpha_{s+1}$. Si $m>0$, alors d'après le lemme 1 , on a $\mathbf{g}_{s+r+1}(m) \in p^{k_{0}} \mathcal{O}$. Donc, on obtient, pour tout $m>0$, que $\mathbf{S}_{r}(a, K, s, p, m) \in p^{s+k_{0}+1} \mathcal{O}$. D'où également, $\mathbf{S}_{r}(a, K, s, p, 0)=-\sum_{m=1}^{T} \mathbf{S}_{r}(a, K, s, p, m) \in p^{s+k_{0}+1} \mathcal{O}$. De plus, d'après les conditions (i) et $(i i)$, pour tout $m \in \mathbb{N}$ et tout $r \geq 0$, on a $\frac{\mathbf{A}_{s+r+1}\left(m p^{k_{0}}\right)}{\mathbf{A}_{s+r+1}(0)} \in \mathbf{g}_{s+r+1}\left(m p^{k_{0}}\right) \mathcal{O}$. Ainsi, d'après $\beta_{s, s}$ (i.e. (2.16) ), pour tout $m \in \mathbb{N}$ et tout $K \in \mathbb{Z}$, on obtient $\mathbf{S}_{r}\left(a, K+m p^{s+k_{0}}, s, p, m p^{k_{0}}\right) \in$ $p^{s+k_{0}+1} \mathbf{g}_{s+r+1}\left(m p^{k_{0}}\right) \mathcal{O}$ et donc $\alpha_{s+1}$ est vraie. Ceci achève la récurrence sur $s$. Ainsi, pour tout $s \in \mathbb{N}, s \geq 1, \alpha_{s}$ est vraie et, en particulier, $\mathbf{S}_{r}(a, K, s, p, m) \in p^{s+1} \mathbf{g}_{s+r+1}(m) \mathcal{O}$. Il ne reste plus qu'à démontrer les lemmes 4, 5] et 6.

Démonstration du lemme 4. En appliquant le lemme 2 avec $k=0$ et $k=k_{0}$, on obtient respectivement $\mathbf{S}_{r}(a, K, 0, p, m) \in p \mathbf{g}_{r+1}(m) \mathcal{O}$ et $\mathbf{S}_{r}\left(a, K, 0, p, m p^{k_{0}}\right) \in p^{k_{0}+1} \mathbf{g}_{r+1}\left(m p^{k_{0}}\right) \mathcal{O}$, ce qui n'est rien d'autre que l'assertion $\alpha_{1}$ et termine la preuve du lemme 4 . 
Démonstration du lemme 5. On a

$$
\begin{aligned}
\mathbf{U}_{r}(a, K+ & \left.m p^{s+k_{0}}, p, j+m p^{s+k_{0}}\right)-\frac{\mathbf{A}_{r+1}\left(j+m p^{s+k_{0}}\right)}{\mathbf{A}_{r+1}(j)} \mathbf{U}_{r}(a, K, p, j) \\
& =-\mathbf{A}_{r+1}(K-j) \mathbf{A}_{r}(a+j p)\left(\frac{\mathbf{A}_{r}\left(a+j p+m p^{s+k_{0}+1}\right)}{\mathbf{A}_{r}(a+j p)}-\frac{\mathbf{A}_{r+1}\left(j+m p^{s+k_{0}}\right)}{\mathbf{A}_{r+1}(j)}\right) .
\end{aligned}
$$

Comme $a<p, j<p^{s}$ et $v_{p}\left(m p^{k_{0}}\right) \geq k_{0}$, l'hypothèse (iii) implique que le terme de droite de l'égalité (2.19) est dans $\mathbf{A}_{r+1}(K-j) \mathbf{A}_{r}(a+j p) p^{s+k_{0}+1} \frac{\mathbf{g}_{s+r+1}\left(m p^{k}\right)}{\mathbf{g}_{r}(a+j p)} \mathcal{O}$. De plus, d'après la condition $(i i)$, on a $\mathbf{A}_{r}(a+j p) \in \mathbf{g}_{r}(a+j p) \mathcal{O}$ et $\mathbf{A}_{r+1}(K-j) \in \mathbf{g}_{r+1}(K-j) \mathcal{O} \subset \mathcal{O}$. Ces estimations montrent que le membre de gauche de (2.19) est dans $p^{s+k_{0}+1} \mathbf{g}_{s+r+1}\left(m p^{k_{0}}\right) \mathcal{O}$, comme voulu.

Démonstration du lemme [6. Pour $t<s$, on écrit $\beta_{t, s}$ sous la forme

$$
\begin{aligned}
& \mathbf{S}_{r}\left(a, K+m p^{s+k_{0}}, s, p, m p^{k_{0}}\right) \equiv \\
& \sum_{i=0}^{p-1} \sum_{\mu=0}^{p^{s-t-1}-1} \frac{\mathbf{A}_{t+r+1}\left(i+\mu p+m p^{s-t+k_{0}}\right)}{\mathbf{A}_{t+r+1}(i+\mu p)} \mathbf{S}_{r}(a, K, t, p, i+\mu p) \quad \bmod p^{s+k_{0}+1} \mathbf{g}_{s+r+1}\left(m p^{k_{0}}\right) \mathcal{O} .
\end{aligned}
$$

On veut montrer $\beta_{t+1, s}$, qui s'écrit

$$
\begin{aligned}
\mathbf{S}_{r}\left(a, K+m p^{s+k_{0}}, s, p, m p^{k_{0}}\right) \equiv \\
\sum_{\mu=0}^{p^{s-t-1}-1} \frac{\mathbf{A}_{t+r+2}\left(\mu+m p^{s-t+k_{0}-1}\right)}{\mathbf{A}_{t+r+2}(\mu)} \mathbf{S}_{r}(a, K, t+1, p, \mu) \quad \bmod p^{s+k_{0}+1} \mathbf{g}_{s+r+1}\left(m p^{k_{0}}\right) \mathcal{O} .
\end{aligned}
$$

On remarque que $\mathbf{S}_{r}(a, K, t+1, p, \mu)=\sum_{i=0}^{p-1} \mathbf{S}_{r}(a, K, t, p, i+\mu p)$. Ainsi, en posant

$$
X:=\mathbf{S}_{r}\left(a, K+m p^{s+k_{0}}, s, p, m p^{k_{0}}\right)-\sum_{\mu=0}^{p^{s-t-1}-1} \frac{\mathbf{A}_{t+r+2}\left(\mu+m p^{s-t+k_{0}-1}\right)}{\mathbf{A}_{t+r+2}(\mu)} \sum_{i=0}^{p-1} \mathbf{S}_{r}(a, K, t, p, i+\mu p),
$$

il ne reste plus qu'à montrer que $X \in p^{s+k_{0}+1} \mathbf{g}_{s+r+1}\left(m p^{k_{0}}\right) \mathcal{O}$. D'après $\beta_{t, s}$ sous la forme (2.20), on obtient que

$$
\begin{aligned}
X & \equiv \sum_{i=0}^{p-1} \sum_{\mu=0}^{p^{s-t-1}-1} \mathbf{S}_{r}(a, K, t, p, i+\mu p) \\
& \times\left(\frac{\mathbf{A}_{t+r+1}\left(i+\mu p+m p^{s-t+k_{0}}\right)}{\mathbf{A}_{t+r+1}(i+\mu p)}-\frac{\mathbf{A}_{t+r+2}\left(\mu+m p^{s-t-1+k_{0}}\right)}{\mathbf{A}_{t+r+2}(\mu)}\right) \quad \bmod p^{s+k_{0}+1} \mathbf{g}_{s+r+1}\left(m p^{k_{0}}\right) \mathcal{O} .
\end{aligned}
$$

Or, d'après l'hypothèse $($ iii $)$ appliquée avec $s-t-1$ pour $s$ et $m p^{k_{0}}$ pour $m$, on a

$$
\frac{\mathbf{A}_{t+r+1}\left(i+\mu p+m p^{s-t+k_{0}}\right)}{\mathbf{A}_{t+r+1}(i+\mu p)}-\frac{\mathbf{A}_{t+r+2}\left(\mu+m p^{s-t-1+k_{0}}\right)}{\mathbf{A}_{t+r+2}(\mu)} \in p^{s-t+k_{0}} \frac{\mathbf{g}_{s+r+1}\left(m p^{k_{0}}\right)}{\mathbf{g}_{t+r+1}(i+\mu p)} \mathcal{O} .
$$

De plus, comme $t<s$ et puisque $\alpha_{s}$ est vraie, on a $\mathbf{S}_{r}(a, K, t, p, i+\mu p) \in p^{t+1} \mathbf{g}_{t+r+1}(i+\mu p) \mathcal{O}$. Donc on a bien $X \in p^{s+k_{0}+1} \mathbf{g}_{s+r+1}\left(m p^{k_{0}}\right) \mathcal{O}$. Ceci achève la preuve du lemme 6 et donc celle du théorème 3 . 


\section{Un ÉNONCÉ $p$-ADIQUE ÉQUIVALENT AU CRITÈRE}

On se place sous les hypothèses des théorèmes 1 et 2 . On fixe $L \in\left\{1, \ldots, M_{(\mathbf{e}, \mathbf{f})}\right\}$ dans cette partie. On rappelle que $q_{(\mathbf{e}, \mathbf{f})} \in z \mathbb{Z}[[z]]$, respectivement $q_{L,(\mathbf{e}, \mathbf{f})} \in \mathbb{Z}[[z]]$, si, et seulement si, pour tout nombre premier $p$, on a $q_{(\mathbf{e}, \mathbf{f})} \in z \mathbb{Z}_{p}[[z]]$, respectivement $q_{L,(\mathbf{e}, \mathbf{f})} \in \mathbb{Z}_{p}[[z]]$.

Nous allons définir, pour tout nombre premier $p$, des éléments $\Phi_{p}(a+K p)$ et $\Phi_{L, p}(a+K p)$ de $\mathbb{Q}_{p}$, où $a \in\{0, \ldots, p-1\}$ et $K \in \mathbb{N}$, et montrer que $q_{(\mathbf{e}, \mathbf{f})} \in z \mathbb{Z}_{p}[[z]]$, respectivement $q_{L,(\mathbf{e}, \mathbf{f})} \in \mathbb{Z}_{p}[[z]]$, si, et seulement si, pour tout nombre premier $p$, tout $a \in\{0, \ldots, p-1\}$ et tout $K \in \mathbb{N}$, on a $\Phi_{p}(a+K p) \in p \mathbb{Z}_{p}$, respectivement $\Phi_{L, p}(a+K p) \in p \mathbb{Z}_{p}$.

Pour alléger les notations, on notera $\Delta:=\Delta_{(\mathbf{e}, \mathbf{f})}, \mathcal{Q}:=\mathcal{Q}_{(\mathbf{e}, \mathbf{f})}, F:=F_{(\mathbf{e}, \mathbf{f})}, G:=G_{(\mathbf{e}, \mathbf{f})}, G_{L}:=$ $G_{L,(\mathbf{e}, \mathbf{f})}, q:=q_{(\mathbf{e}, \mathbf{f})}$ et $q_{L}:=q_{L,(\mathbf{e}, \mathbf{f})}$, comme dans toute la suite de l'article. On fixe un nombre premier $p$ dans cette partie.

Avant de donner les preuves des théorèmes 1 et 2, on va les reformuler. Le résultat classique suivant est dû à Dieudonné et Dwork (voir [4, Chap. VI, Sec. 2, Lemma 3]; 77, Chap. 14, Sec. 2]).

Lemme 7. Soit $F(z)$ une série formelle dans $1+z \mathbb{Q}[[z]]$. Alors $F(z) \in 1+z \mathbb{Z}_{p}[[z]]$ si et seulement si $\frac{F\left(z^{p}\right)}{F(z)^{p}} \in 1+p z \mathbb{Z}_{p}[[z]]$.

On déduit de ce lemme le corollaire suivant (voir [13, Lemma 5, p. 610]), qui va nous permettre «d'éliminer » l'exponentielle dans les expressions $q(z)=z \exp (G(z) / F(z))$ et $q_{L}(z)=$ $\exp \left(G_{L}(z) / F(z)\right)$.

Corollaire 1. Soit $f(z) \in z \mathbb{Q}[[z]]$. On a $e^{f(z)} \in 1+z \mathbb{Z}_{p}[[z]]$ si et seulement si $f\left(z^{p}\right)-p f(z) \in$ $p z \mathbb{Z}_{p}[[z]]$.

D'après les identités (1.1) et (1.2) définissant respectivement $G$ et $G_{L}$, on a $G(0)=G_{L}(0)=0$ et donc $G(z) / F(z)$ et $G_{L}(z) / F(z)$ sont dans $z \mathbb{Q}[[z]]$. Ainsi, d'après le corollaire[1, on a $q(z) \in z \mathbb{Z}_{p}[[z]]$, respectivement $q_{L}(z) \in \mathbb{Z}_{p}[[z]]$, si, et seulement si $\frac{G}{F}\left(z^{p}\right)-p \frac{G}{F}(z) \in p z \mathbb{Z}_{p}[[z]]$, respectivement $\frac{G_{L}}{F}\left(z^{p}\right)-p \frac{G_{L}}{F}(z) \in p z \mathbb{Z}_{p}[[z]]$.

Or, comme $\mathcal{Q}$ est une suite à termes entiers, on a $F(z) \in 1+z \mathbb{Z}[[z]] \subset 1+z \mathbb{Z}_{p}[[z]]$. Ainsi, $q(z) \in z \mathbb{Z}_{p}[[z]]$, respectivement $q_{L}(z) \in \mathbb{Z}_{p}[[z]]$, si, et seulement si on a $F(z) G\left(z^{p}\right)-p F\left(z^{p}\right) G(z) \in$ $p z \mathbb{Z}_{p}[[z]]$, respectivement $F(z) G_{L}\left(z^{p}\right)-p F\left(z^{p}\right) G_{L}(z) \in p z \mathbb{Z}_{p}[[z]]$.

D'après l'identité (1.1) définissant $G$, le coefficient de $z^{a+K p}$ dans $F(z) G\left(z^{p}\right)-p F\left(z^{p}\right) G(z)$ est

$$
\begin{aligned}
& \Phi_{p}(a+K p):= \\
& \quad \sum_{j=0}^{K} \mathcal{Q}(K-j) \mathcal{Q}(a+j p)\left(\sum_{i=1}^{q_{1}} e_{i}\left(H_{e_{i}(K-j)}-p H_{e_{i}(a+j p)}\right)-\sum_{i=1}^{q_{2}} f_{i}\left(H_{f_{i}(K-j)}-p H_{f_{i}(a+j p)}\right)\right)
\end{aligned}
$$

et, d'après l'identité (1.2) définissant $G_{L}$, le coefficient de $z^{a+K p}$ dans $F(z) G_{L}\left(z^{p}\right)-p F\left(z^{p}\right) G_{L}(z)$ est

$$
\Phi_{L, p}(a+K p):=\sum_{j=0}^{K} \mathcal{Q}(K-j) \mathcal{Q}(a+j p)\left(H_{L(K-j)}-p H_{L(a+j p)}\right) .
$$

On a donc $q(z) \in z \mathbb{Z}_{p}[[z]]$, respectivement $q_{L}(z) \in \mathbb{Z}_{p}[[z]]$, si, et seulement si, pour tout $a \in$ $\{0, \ldots, p-1\}$ et tout $K \in \mathbb{N}$, on a $\Phi_{p}(a+K p) \in p \mathbb{Z}_{p}$, respectivement $\Phi_{L, p}(a+K p) \in p \mathbb{Z}_{p}$.

\section{Démonstration des Cas $(i)$ Des théorèmes 1 et 2}

On se place sous les hypothèses des thèorèmes 1 et [2. On suppose de plus que, pour tout $x \in\left[1 / M_{(\mathbf{e}, \mathbf{f})}, 1[\right.$, on a $\Delta(x) \geq 1$. Comme il a été dit en partie 1.2, le point $(i)$ du théorème 2 entraîne la validité du point $(i)$ du théorème 1. Le but de cette partie est donc de montrer que, pour tout $L \in\left\{1, \ldots, M_{(\mathbf{e}, \mathbf{f})}\right\}$, on a $q_{L}(z) \in \mathbb{Z}[[z]]$. D'après la partie [3, il nous suffit de montrer 
que, pour tout $L \in\left\{1, \ldots, M_{(\mathbf{e}, \mathbf{f})}\right\}$, tout nombre premier $p$, tout $a \in\{0, \ldots, p-1\}$ et tout $K \in \mathbb{N}$, on a $\Phi_{L, p}(a+K p) \in p \mathbb{Z}_{p}$. On fixe $L \in\left\{1, \ldots, M_{(\mathbf{e}, \mathbf{f})}\right\}$ dans cette partie.

4.1. Nouvelle reformulation du problème. Pour tout premier $p$, tout $a \in\{0, \ldots, p-1\}$ et tout $K, s$ et $m$ dans $\mathbb{N}$, on définit

$$
S(a, K, s, p, m):=\sum_{j=m p^{s}}^{(m+1) p^{s}-1}(\mathcal{Q}(a+j p) \mathcal{Q}(K-j)-\mathcal{Q}(j) \mathcal{Q}(a+(K-j) p))
$$

où l'on pose $\mathcal{Q}(\ell)=0$ si $\ell$ est un entier strictement négatif.

Le but de cette partie est de produire, pour tout nombre premier $p$, une fonction $g_{p}$ de $\mathbb{N}$ dans $\mathbb{Z}_{p}$ telle que : si pour tout premier $p$, tout $a \in\{0, \ldots, p-1\}$ et tout $K, s$ et $m$ dans $\mathbb{N}$, on a $S(a, K, s, p, m) \in p^{s+1} g_{p}(m) \mathbb{Z}_{p}$, alors on a $\Phi_{L, p}(a+p K) \in p \mathbb{Z}_{p}$. Démontrer le cas $(i) \mathrm{du}$ théorème 2 reviendra alors à minorer convenablement la valuation $p$-adique de $S(a, K, s, p, m)$ pour tout nombre premier $p$. Cette méthode de réduction est une adaptation de l'approche du problème faite par Dwork dans [3].

4.1.1. Une réécriture de $\Phi_{L, p}(a+K p)$ modulo $p \mathbb{Z}_{p}$. Cette étape est l'analogue d'une réécriture effectuée par Krattenthaler et Rivoal dans la partie 2 de [5]. On fixe un nombre premier $p$. Nous allons montrer que

$$
\Phi_{L, p}(a+K p) \equiv \sum_{j=0}^{K} H_{L j}(\mathcal{Q}(a+j p) \mathcal{Q}(K-j)-\mathcal{Q}(j) \mathcal{Q}(a+(K-j) p)) \quad \bmod p \mathbb{Z}_{p}
$$

Pour tout $a \in\{0, \ldots, p-1\}$ et tout $j \in \mathbb{N}$, on a

$$
\begin{aligned}
p H_{L(a+j p)} & =p\left(\sum_{i=1}^{L j p} \frac{1}{i}+\sum_{i=1}^{L a} \frac{1}{L j p+i}\right) \\
& \equiv p\left(\sum_{i=1}^{L j} \frac{1}{i p}+\sum_{i=1}^{\lfloor L a / p\rfloor} \frac{1}{L j p+i p}\right) \quad \bmod p \mathbb{Z}_{p} \\
& \equiv H_{L j}+\sum_{i=1}^{\lfloor L a / p\rfloor} \frac{1}{L j+i} \bmod p \mathbb{Z}_{p} .
\end{aligned}
$$

Nous avons besoin d'un résultat, que l'on démontrera plus loin dans une forme plus générale (lemme 9, partie 4.1.2) :

Pour tout $L \in\left\{1, \ldots, M_{(\mathbf{e}, \mathbf{f})}\right\}$, tout $a \in\{0, \ldots, p-1\}$ et tout $j \in \mathbb{N}$, on a

$$
\mathcal{Q}(a+j p) \sum_{i=1}^{\lfloor L a / p\rfloor} \frac{1}{L j+i} \in p \mathbb{Z}_{p}
$$


En appliquant (4.3) à (4.2), on obtient $\mathcal{Q}(a+j p) p H_{L(a+j p)} \equiv \mathcal{Q}(a+j p) H_{L j} \bmod p \mathbb{Z}_{p}$ et comme $\mathcal{Q}(K-j) \in \mathbb{Z}_{p}$, cela donne

$$
\begin{aligned}
\Phi_{L, p}(a+K p) & =\sum_{j=0}^{K} \mathcal{Q}(K-j) \mathcal{Q}(a+j p)\left(H_{L(K-j)}-p H_{L(a+j p)}\right) \\
& \equiv \sum_{j=0}^{K} \mathcal{Q}(K-j) \mathcal{Q}(a+j p)\left(H_{L(K-j)}-H_{L j}\right) \quad \bmod p \mathbb{Z}_{p} \\
& \equiv \sum_{j=0}^{K} H_{L j}(\mathcal{Q}(a+j p) \mathcal{Q}(K-j)-\mathcal{Q}(j) \mathcal{Q}(a+(K-j) p)) \quad \bmod p \mathbb{Z}_{p},
\end{aligned}
$$

ce qui est bien l'équation (4.1) attendue.

On utilise maintenant un lemme combinatoire dû à Dwork (voir [3, Lemma 4.2, p. 308]) qui nous permet d'écrire

$$
\sum_{j=0}^{K} H_{L j}(\mathcal{Q}(a+j p) \mathcal{Q}(K-j)-\mathcal{Q}(j) \mathcal{Q}(a+(K-j) p))=\sum_{s=0}^{r} \sum_{m=0}^{p^{r+1-s}-1} W_{L}(a, K, s, p, m),
$$

où $r$ est tel que $K<p^{r}$, et $W_{L}(a, K, s, p, m):=\left(H_{L m p^{s}}-H_{L\lfloor m / p\rfloor p^{s+1}}\right) S(a, K, s, p, m)$. Si l'on montre que, pour tout $m$ et $s$ dans $\mathbb{N}$, on a $W_{L}(a, K, s, p, m) \in p \mathbb{Z}_{p}$, alors on aura bien $\Phi_{L, p}(a+K p) \in p \mathbb{Z}_{p}$, comme voulu.

Dans la suite de l'article, on notera $\{\cdot\}$ la fonction partie fractionnaire. Pour tout $m \in \mathbb{N}$, on pose $\mu_{p}(m):=\sum_{\ell=1}^{\infty} \mathbf{1}_{\left[1 / M_{(\mathbf{e}, \mathbf{f})}, 1[\right.}\left(\left\{m / p^{\ell}\right\}\right)$, où $\mathbf{1}_{\left[1 / M_{(\mathbf{e}, \mathbf{f})}, 1[\right.}$ est la fonction caractéristique de $\left[1 / M_{(\mathbf{e}, \mathbf{f})}, 1[\right.$. Pour tout $m \in \mathbb{N}$, on pose $g_{p}(m):=p^{\mu_{p}(m)}$. On utilise maintenant le lemme suivant que l'on démontre dans la partie 4.1.2.

Lemme 8. Pour tout nombre premier $p$, tout $L \in\left\{1, \ldots, M_{(\boldsymbol{e}, \boldsymbol{f})}\right\}$ et tout $s$ et $m$ dans $\mathbb{N}$, on a

$$
p^{s+1} g_{p}(m)\left(H_{L m p^{s}}-H_{L\lfloor m / p\rfloor p^{s+1}}\right) \in p \mathbb{Z}_{p} .
$$

D'après le lemme [8, si on montre que, pour tout $a \in\{0, \ldots, p-1\}$, tout $K, s$ et $m$ dans $\mathbb{N}$, on a $S(a, K, s, p, m) \in p^{s+1} g_{p}(m) \mathbb{Z}_{p}$, alors, on aura $q_{L}(z) \in \mathbb{Z}_{p}[[z]]$, ce qui est la reformulation annoncée.

4.1.2. Démonstration de (4.3) et du lemme 8. Nous allons énoncer un résultat plus général permettant de démontrer le résultat (4.3) et le lemme 8 .

Lemme 9. Soit $s \in \mathbb{N}, s \geq 1, a \in\left\{0, \ldots, p^{s}-1\right\}, M \geq 1$ et $m \in \mathbb{N}$. Soit $L \in\{1, \ldots, M\}$. Si $\left\lfloor L a / p^{s}\right\rfloor \geq 1$ alors, pour tout $u \in\left\{1, \ldots,\left\lfloor L a / p^{s}\right\rfloor\right\}$ et tout $\ell \in\left\{s, \ldots, s+v_{p}(L m+u)\right\}$, on a $\left\{\frac{a+m p^{s}}{p^{\ell}}\right\} \geq \frac{1}{M}$.

Démonstration. On note $m=\sum_{j=0}^{\infty} m_{j} p^{j}$ le développement $p$-adique de $m$. On a

$$
\left\{\frac{a+m p^{s}}{p^{\ell}}\right\}=\frac{a+p^{s} \sum_{j=0}^{\ell-s-1} m_{j} p^{j}}{p^{\ell}}
$$

On a $p^{\ell-s} \mid(u+L m)$ et donc $p^{\ell-s} \mid\left(u+L m-L\left(\sum_{j=\ell-s}^{\infty} m_{j} p^{j}\right)\right)=\left(u+L\left(\sum_{j=0}^{\ell-s-1} m_{j} p^{j}\right)\right)$. Ainsi, on obtient

$$
p^{\ell-s} \leq u+L\left(\sum_{j=0}^{\ell-s-1} m_{j} p^{j}\right) \leq \frac{1}{p^{s}} L a+L\left(\sum_{j=0}^{\ell-s-1} m_{j} p^{j}\right)=\frac{L}{p^{s-\ell}}\left\{\frac{a+m p^{s}}{p^{\ell}}\right\} \leq \frac{M}{p^{s-\ell}}\left\{\frac{a+m p^{s}}{p^{\ell}}\right\}
$$

et on a bien $\left\{\frac{a+m p^{s}}{p^{\ell}}\right\} \geq \frac{1}{M}$. 
Nous allons maintenant appliquer le lemme 9 pour démontrer (4.3) en utilisant le fait que, pour tout $n \in \mathbb{N}$, on a $v_{p}(\mathcal{Q}(n))=\sum_{\ell=1}^{\infty} \Delta\left(\left\{\frac{n}{p^{\ell}}\right\}\right)$. En effet, pour tout entier positif $c$, on a $\lfloor c x\rfloor=$ $\lfloor c\{x\}\rfloor+c\lfloor x\rfloor$ et donc $\Delta(x)=\Delta(\{x\})+(|\mathbf{e}|-|\mathbf{f}|)\lfloor x\rfloor$. Ainsi, on a $|\mathbf{e}|=|\mathbf{f}|$ si et seulement si $\Delta$ est 1-périodique. On rappelle que si $m$ est un entier naturel, on a la formule $v_{p}((m) !)=\sum_{\ell=1}^{\infty}\left\lfloor\frac{m}{p^{\ell}}\right\rfloor$. Ainsi, on obtient bien

$$
v_{p}(\mathcal{Q}(n))=v_{p}\left(\frac{\left(e_{1} n\right) ! \cdots\left(e_{q_{1}} n\right) !}{\left(f_{1} n\right) ! \cdots\left(f_{q_{2}} n\right) !}\right)=\sum_{\ell=1}^{\infty} \Delta\left(\frac{n}{p^{\ell}}\right)=\sum_{\ell=1}^{\infty} \Delta\left(\left\{\frac{n}{p^{\ell}}\right\}\right) .
$$

Démonstration de (4.3). Soit $L \in\left\{1, \ldots, M_{(\mathbf{e}, \mathbf{f})}\right\}, a \in\{0, \ldots, p-1\}$ et $j \in \mathbb{N}$. Il faut montrer que $\mathcal{Q}(a+j p) \sum_{i=1}^{\lfloor L a / p\rfloor} \frac{1}{L j+i} \in p \mathbb{Z}_{p}$. Si $\lfloor L a / p\rfloor=0$, c'est évident. Supposons que $\lfloor L a / p\rfloor \geq 1$. En appliquant le lemme 9 avec $s=1, m=j$ et $M=M_{(\mathbf{e}, \mathbf{f})}$, on obtient que, pour tout $i \in$ $\{1, \ldots,\lfloor L a / p\rfloor\}$ et tout $\ell \in\left\{1, \ldots, 1+v_{p}(i+L j)\right\}$, on a $\left\{(a+j p) / p^{\ell}\right\} \geq 1 / M_{(\mathbf{e}, \mathbf{f})}$ et donc $\Delta(\{(a+$ $\left.\left.j p) / p^{\ell}\right\}\right) \geq 1$. Comme $\Delta$ est positive sur $\mathbb{R}$, cela donne

$$
v_{p}(\mathcal{Q}(a+j p))=\sum_{\ell=1}^{\infty} \Delta\left(\left\{\frac{a+j p}{p^{\ell}}\right\}\right) \geq \sum_{\ell=1}^{1+v_{p}(L j+i)} \Delta\left(\left\{\frac{a+j p}{p^{\ell}}\right\}\right) \geq 1+v_{p}(L j+i),
$$

ce qui achève la preuve de (4.3).

Démonstration du lemme 8 . Soit $L \in\left\{1, \ldots, M_{(\mathbf{e}, \mathbf{f})}\right\}$ et $m$ et $s$ dans $\mathbb{N}$. Il faut montrer que $p^{s+1} g_{p}(m)\left(H_{L m p^{s}}-H_{L\lfloor m / p\rfloor p^{s+1}}\right) \in p \mathbb{Z}_{p}$. On écrit $m=b+q p$, où $b \in\{0, \ldots, p-1\}$ et $q \in \mathbb{N}$. On a alors $L m p^{s}=L b p^{s}+L q p^{s+1}$ et $L\lfloor m / p\rfloor p^{s+1}=L q p^{s+1}$. Ainsi, on obtient

$$
H_{L m p^{s}}-H_{L\lfloor m / p\rfloor p^{s+1}}=\sum_{j=1}^{L b p^{s}} \frac{1}{L q p^{s+1}+j} \equiv \sum_{i=1}^{\lfloor L b / p\rfloor} \frac{1}{L q p^{s+1}+i p^{s+1}} \quad \bmod \frac{1}{p^{s}} \mathbb{Z}_{p}
$$

et donc $p^{s+1} g_{p}(m)\left(H_{L m p^{s}}-H_{L\lfloor m / p\rfloor p^{s+1}}\right) \equiv g_{p}(b+q p) \sum_{i=1}^{\lfloor L b / p\rfloor} \frac{1}{L q+i} \bmod p \mathbb{Z}_{p}$. Il nous reste à montrer que $g_{p}(b+q p) \sum_{i=1}^{\lfloor L b / p\rfloor} \frac{1}{L q+i} \in p \mathbb{Z}_{p}$. Si $\lfloor L b / p\rfloor=0$, c'est évident. Supposons que $\lfloor L b / p\rfloor \geq 1$. En appliquant le lemme 9 avec $s=1, M=M_{(\mathbf{e}, \mathbf{f})}, a=b$ et $q$ à la place de $m$, on obtient que, pour tout $i \in\{1, \ldots,\lfloor L b / p\rfloor\}$ et tout $\ell \in\left\{1, \ldots, 1+v_{p}(i+L q)\right\}$, on a $\left\{(b+q p) / p^{\ell}\right\} \geq 1 / M_{(\mathbf{e}, \mathbf{f})}$ et donc

$v_{p}\left(g_{p}(b+q p)\right)=\sum_{\ell=1}^{\infty} \mathbf{1}_{\left[1 / M_{(\mathbf{e}, \mathbf{f})}, 1[\right.}\left(\left\{\frac{b+q p}{p^{\ell}}\right\}\right) \geq \sum_{\ell=1}^{1+v_{p}(L q+i)} \mathbf{1}_{\left[1 / M_{(\mathbf{e}, \mathbf{f})}, 1[\right.}\left(\left\{\frac{b+q p}{p^{\ell}}\right\}\right) \geq 1+v_{p}(L q+i)$,

ce qui achève la preuve du lemme.

4.2. Application du théorème 3. La stratégie utilisée par Krattenthaler et Rivoal dans [5] est d'appliquer le critère de Dwork (cas $k_{0}=0$ du théorème 3) avec le choix des fonctions $A_{r}=g_{r}=\mathcal{Q}$ pour tout $r \geq 0$. Cette stratégie ne marche cependant pas toujours quand $\Delta$ n'est pas croissante sur $[0,1]$, même si on ne prend pas forcément $A_{r}=g_{r}=\mathcal{Q}$ pour tout $r \geq 0$. En effet, si on dispose de deux suites $\left(A_{r}\right)_{r \geq 0}$ et $\left(g_{r}\right)_{r \geq 0}$ vérifiant les conditions du critère de Dwork et telles qu'il existe un $r \geq 0$ tel que $A_{r}=\bar{A}_{r+1}=\mathcal{Q}$ et, pour tout $s, r$ et $m$ dans $\mathbb{N}, p^{s+1} g_{s+r+1}(m)\left(H_{L m p^{s}}-H_{L\lfloor m / p\rfloor p^{s+1}}\right) \in$ $\mathcal{O}$, alors (iii) nous dit que, pour tout nombre premier $p$, tout $v<p$, tout $s, r$ et $m$ dans $\mathbb{N}$ et tout $u<p^{s}$, on a

$$
\left(H_{L m p^{s}}-H_{L\lfloor m / p\rfloor p^{s+1}}\right)\left(\frac{\mathcal{Q}\left(v+u p+m p^{s+1}\right)}{\mathcal{Q}(v+u p)}-\frac{\mathcal{Q}\left(u+m p^{s}\right)}{\mathcal{Q}(u)}\right) \in \frac{1}{\mathbf{g}_{r}(v+u p)} \mathcal{O} \subset \frac{1}{\mathcal{Q}(v+u p)} \mathcal{O} .
$$


Or (4.4) n'est pas vérifiée par la suite $\mathcal{Q}_{(\mathbf{e}, \mathbf{f})}$ définie par $\mathbf{e}=(10,5)$ et $\mathbf{f}=(4,4,3,2,1,1)$. En effet, pour $p=3, L=10, v=1, s=1, m=1$ et $u=2$ on obtient

$$
v_{3}\left(H_{30}\left(\frac{\mathcal{Q}_{(\mathbf{e}, \mathbf{f})}(16)}{\mathcal{Q}_{(\mathbf{e}, \mathbf{f})}(7)}-\frac{\mathcal{Q}_{(\mathbf{e}, \mathbf{f})}(5)}{\mathcal{Q}_{(\mathbf{e}, \mathbf{f})}(2)}\right)\right)=-4 \quad \text { et } \quad v_{3}\left(\frac{1}{\mathcal{Q}_{(\mathbf{e}, \mathbf{f})}(7)}\right)=-3 .
$$

Précisons la manière dont nous allons utiliser le théorème 3 pour terminer la démonstration du cas $(i)$ du théorème 2, Nous allons montrer dans les parties suivantes qu'il existe un entier naturel $\lambda_{p}$ tel qu'en posant $\mathbf{A}_{r}=\mathcal{Q}$ et $\mathbf{g}_{r}=g_{p}$ pour tout $r \geq 0,\left(\left(\mathbf{A}_{r}\right)_{r \geq 0},\left(\mathbf{g}_{r}\right)_{r \geq 0}\right)$ est un $\lambda_{p}$-couple de Dwork. En appliquant alors le théorème [3, on obtiendra bien $S(a, K, s, p, m) \in p^{s+1} g_{p}(m) \mathbb{Z}_{p}$, comme voulu.

Dans les parties suivantes, on vérifie les hypothèses d'application du théorème 3 .

4.3. Vérification des conditions $(i),(i i)$ et $(i v)$ du théorème 3. On fixe $p$ un nombre premier et on note $g:=g_{p}$ et $\mu:=\mu_{p}$. Pour tout $r \geq 0$, on pose $\mathbf{A}_{r}=\mathcal{Q}$ et $\mathbf{g}_{r}=g$. On définit $\lambda_{p}$ comme étant l'unique entier naturel vérifiant $p^{\lambda_{p}}<M_{(\mathbf{e}, \mathbf{f})} \leq p^{\lambda_{p}+1}$. On va montrer dans cette partie que les suites $\left(\mathbf{A}_{r}\right)_{r \geq 0}$ et $\left(\mathbf{g}_{r}\right)_{r \geq 0}$ vérifient les conditions $(i)$, $(i i)$ et $(i v)$ du théorème 3 avec $k_{0}=\lambda_{p}$. Pour cela, nous allons uniquement nous servir de l'inégalité $p^{\lambda_{p}}<M_{(\mathbf{e}, \mathbf{f}) \text {. L'inégalité }} M_{(\mathbf{e}, \mathbf{f})} \leq p^{\lambda_{p}+1}$ nous servira à démontrer la condition (iii) du théorème 3 dans la partie suivante.

- Vérification de $(i)$ et $(i i)$.

Pour tout $r$ et $m$ dans $\mathbb{N}$, on a $\left|\mathbf{A}_{r}(0)\right|_{p}=|\mathcal{Q}(0)|_{p}=1$. De plus, $v_{p}\left(\mathbf{g}_{r}(m)\right)=\mu(m) \geq 0$, donc on a bien $\mathbf{g}_{r}(m) \in \mathbb{Z}_{p} \backslash\{0\}$. Il ne reste plus qu'à montrer que $\mathbf{A}_{r}(m) \in \mathbf{g}_{r}(m) \mathbb{Z}_{p}$, ce qui revient donc à montrer qu'on a $\mu(m) \leq v_{p}(\mathcal{Q}(m))$. C'est bien le cas puisque, pour tout $\ell \in \mathbb{N}, \ell \geq 1$, on a $\Delta\left(\left\{\frac{m}{p^{\ell}}\right\}\right) \geq 1_{\left[1 / M_{(\mathbf{e}, \mathbf{f})}, 1[\right.}\left(\left\{\frac{m}{p^{\ell}}\right\}\right)$, car $\Delta(x) \geq 1$ pour $1>x \geq 1 / M_{(\mathbf{e}, \mathbf{f})}$. On obtient bien $v_{p}(\mathcal{Q}(m))=\sum_{\ell=1}^{\infty} \Delta\left(\left\{\frac{m}{p^{\ell}}\right\}\right) \geq \sum_{\ell=1}^{\infty} 1_{\left[1 / M_{(\mathbf{e}, \mathbf{f})}, 1[\right.}\left(\left\{\frac{m}{p^{\ell}}\right\}\right)=\mu(m)$. D'où le résultat.

- Vérification de $(i v)$ avec $k_{0}=\lambda_{p}$.

Si $\lambda_{p}=0$, il n'y a rien à vérifier. Supposons $\lambda_{p} \geq 1$. Soit $k \in\left\{1, \ldots, \lambda_{p}\right\}, v \in\{1, \ldots, p-1\}$ et $m \in \mathbb{N}$. On a $p^{\lambda_{p}}<M_{(\mathbf{e}, \mathbf{f})}$ donc $p^{k}<M_{(\mathbf{e}, \mathbf{f})}$. Ainsi $1 / M_{(\mathbf{e}, \mathbf{f})}<1 / p^{k}$ et donc, pour tout $\ell \in\{1, \ldots, k\}$, on a $1_{\left[1 / M_{(\mathbf{e}, \mathbf{f})}, 1[\right.}\left(\left\{\frac{v+m p^{k}}{p^{\ell}}\right\}\right)=1_{\left[1 / M_{(\mathbf{e}, \mathbf{f})}, 1[\right.}\left(\left\{\frac{v}{p^{\ell}}\right\}\right)=1$. On a alors

$$
v_{p}\left(g\left(v+m p^{k}\right)\right)=\sum_{\ell=1}^{\infty} 1_{\left[1 / M_{(\mathbf{e}, \mathbf{f})}, 1[\right.}\left(\left\{\frac{v+m p^{k}}{p^{\ell}}\right\}\right)=k+\sum_{\ell=k+1}^{\infty} 1_{\left[1 / M_{(\mathbf{e}, \mathbf{f})}, 1[\right.}\left(\left\{\frac{v+m p^{k}}{p^{\ell}}\right\}\right) .
$$

Pour tout $\ell \geq k+1$, on a $\left\{\frac{v+m p^{k}}{p^{\ell}}\right\}>\left\{\frac{m p^{k}}{p^{\ell}}\right\}$. En effet, on écrit $m=c p^{\ell-k}+d$ où $c \in \mathbb{N}$ et $d \in\left\{0, \ldots, p^{\ell-k}-1\right\}$, et on obtient bien $\left\{\frac{v+m p^{k}}{p^{\ell}}\right\}=\left\{\frac{v+d p^{k}}{p^{\ell}}\right\}=\frac{v+d p^{k}}{p^{\ell}}>\frac{d p^{k}}{p^{\ell}}=\left\{\frac{d p^{k}}{p^{\ell}}\right\}=\left\{\frac{m p^{k}}{p^{\ell}}\right\}$.

Donc $v_{p}\left(g\left(v+m p^{k}\right)\right) \geq k+\sum_{\ell=k+1}^{\infty} 1_{\left[1 / M_{(\mathbf{e}, \mathbf{f})}, 1[\right.}\left(\left\{\frac{m p^{k}}{p^{\ell}}\right\}\right)=k+v_{p}\left(g\left(m p^{k}\right)\right)$ et on a bien $g(v+$ $\left.m p^{k}\right) \in p^{k} g\left(m p^{k}\right) \mathbb{Z}_{p}$. De plus,

$$
\begin{aligned}
v_{p}\left(g\left(m p^{k}\right)\right) & =\sum_{\ell=1}^{\infty} 1_{\left[1 / M_{(\mathbf{e}, \mathbf{f})}, 1[\right.}\left(\left\{\frac{m p^{k}}{p^{\ell}}\right\}\right)=\sum_{\ell=k+1}^{\infty} 1_{\left[1 / M_{(\mathbf{e}, \mathbf{f})}, 1[\right.}\left(\left\{\frac{m p^{k}}{p^{\ell}}\right\}\right) \\
& =\sum_{\ell=1}^{\infty} 1_{\left[1 / M_{(\mathbf{e}, \mathbf{f})}, 1[\right.}\left(\left\{\frac{m}{p^{\ell}}\right\}\right)=v_{p}(g(m)),
\end{aligned}
$$

donc $g\left(m p^{k}\right) \in g(m) \mathbb{Z}_{p}$, comme voulu. 
4.4. Vérification de la condition $($ iii $)$ du théorème 3. On rappelle que $\lambda_{p}$ est l'unique entier naturel vérifiant $p^{\lambda_{p}}<M_{(\mathbf{e}, \mathbf{f})} \leq p^{\lambda_{p}+1}$. On va montrer que les suites $\left(\mathbf{A}_{r}\right)_{r \geq 0}$ et $\left(\mathbf{g}_{r}\right)_{r \geq 0}$ vérifient la condition $($ iii $)$ du théorème 3 avec $k_{0}=\lambda_{p}$. Pour cela, nous n'utiliserons que l'inégalité $M_{(\mathbf{e}, \mathbf{f})} \leq p^{\lambda_{p}+1}$. D'après la partie précédente, la vérification de la condition (iii) montrera que $\left(\left(\mathbf{A}_{r}\right)_{r \geq 0},\left(\mathbf{g}_{r}\right)_{r \geq 0}\right)$ est un $\lambda_{p}$-couple de Dwork et ainsi achèvera la preuve du point $(i)$ du théorème 2, Nous allons montrer que la condition $(i i i)$ est vérifiée en deux étapes, selon que $v_{p}(m) \geq \lambda_{p}$ ou que $v_{p}(m) \leq \lambda_{p}-1$. La preuve est relativement longue et décomposée en nombreuses étapes.

4.4.1. Lorsque $v_{p}(m) \leq \lambda_{p}-1$. Nous devons montrer ici que si $\lambda_{p} \geq 1$ et si $v_{p}(m)=k \leq \lambda_{p}-1$, alors on a $\frac{\mathcal{Q}(m p)}{\mathcal{Q}(0)}-\frac{\mathcal{Q}(m)}{\mathcal{Q}(0)} \in p^{k+1} g(m) \mathbb{Z}_{p}$. Comme $\mathcal{Q}(0)=1$, cela revient à montrer que l'on a

$$
\mathcal{Q}(m)\left(\frac{\mathcal{Q}(m p)}{\mathcal{Q}(m)}-1\right) \in p^{k+1} g(m) \mathbb{Z}_{p}
$$

On écrit $m=p^{k} m^{\prime}$, où $m^{\prime} \in \mathbb{N}$ et (4.5) devient

$$
\mathcal{Q}(m)\left(\frac{\mathcal{Q}\left(m^{\prime} p^{k+1}\right)}{\mathcal{Q}\left(m^{\prime} p^{k}\right)}-1\right) \in p^{k+1} g(m) \mathbb{Z}_{p} .
$$

Pour conclure, on utilise le lemme suivant.

Lemme 10. Pour tout $s \in \mathbb{N}$, tout $c \in\left\{0, \ldots, p^{s}-1\right\}$ et tout $m \in \mathbb{N}$, on a

$$
\frac{\mathcal{Q}(c)}{\mathcal{Q}(c p)} \frac{\mathcal{Q}\left(c p+m p^{s+1}\right)}{\mathcal{Q}\left(c+m p^{s}\right)} \in 1+p^{s+1} \mathbb{Z}_{p}
$$

En appliquant le lemme 10 avec $s=k, c=0$ et $m^{\prime}$ à la place de $m$, on obtient $\frac{\mathcal{Q}\left(m^{\prime} p^{k+1}\right)}{\mathcal{Q}\left(m^{\prime} p^{k}\right)} \in$ $1+p^{k+1} \mathbb{Z}_{p}$ et donc $\left(\frac{\mathcal{Q}\left(m^{\prime} p^{k+1}\right)}{\mathcal{Q}\left(m^{\prime} p^{k}\right)}-1\right) \in p^{k+1} \mathbb{Z}_{p}$. De plus, d'après la condition $(i i)$ du théorème 3 , on a $\mathcal{Q}(m) \in g(m) \mathbb{Z}_{p}$, donc on a bien (4.6). Il ne nous reste plus qu'à démontrer le lemme 10. Pour cela, nous allons utiliser certaines propriétés de la fonction gamma $p$-adique définie par $\Gamma_{p}(n):=(-1)^{n} \gamma_{p}(n)$, où $\gamma_{p}(n):=\prod_{(k, p)=1}^{n-1} k$. On peut étendre $\Gamma_{p}$ à tout $\mathbb{Z}_{p}$ mais on n'en aura pas besoin ici. On résume les propriétés qui nous serviront pour prouver le lemme 10.

Lemme 11. (i) Pour tout $n \in \mathbb{N}$, on a l'identité $\frac{(n p) !}{n !}=p^{n} \gamma_{p}(1+n p)$.

(ii) Pour tout $k, n$ et $s$ dans $\mathbb{N}$, on $a \Gamma_{p}\left(k+n p^{s}\right) \equiv \Gamma_{p}(k) \bmod p^{s}$.

Le point $(i)$ du lemme11 s'obtient en remarquant que $\gamma_{p}(1+n p)=\frac{(n p) !}{n ! p^{n}}$. Le point $(i i)$ du lemme 11 est le lemme 1.1 de [7].

Démonstration du lemme 10. On a

$$
\begin{aligned}
\frac{\mathcal{Q}\left(c p+m p^{s+1}\right)}{\mathcal{Q}\left(c+m p^{s}\right)} & =\prod_{i=1}^{q_{1}} \frac{\left(e_{i}\left(c p+m p^{s+1}\right)\right) !}{\left(e_{i}\left(c+m p^{s}\right)\right) !} \prod_{i=1}^{q_{2}} \frac{\left(f_{i}\left(c+m p^{s}\right)\right) !}{\left(f_{i}\left(c p+m p^{s+1}\right)\right) !} \\
& =\left(\prod_{i=1}^{q_{1}} p^{e_{i}\left(c+m p^{s}\right)} \gamma_{p}\left(1+e_{i} c p+e_{i} m p^{s+1}\right)\right)\left(\prod_{i=1}^{q_{2}} \frac{1}{p^{f_{i}\left(c+m p^{s}\right)} \gamma_{p}\left(1+f_{i} c p+f_{i} m p^{s+1}\right)}\right) \\
& =\left(p^{m p^{s}}\right)^{(|\mathbf{e}|-|\mathbf{f}|)} \frac{\prod_{i=1}^{q_{1}}\left(p^{e_{i} c}(-1)^{1+e_{i} c p+e_{i} m p^{s+1}} \Gamma_{p}\left(1+e_{i} c p+e_{i} m p^{s+1}\right)\right)}{\prod_{i=1}^{q_{2}}\left(p^{f_{i} c}(-1)^{1+f_{i} c p+f_{i} m p^{s+1}} \Gamma_{p}\left(1+f_{i} c p+f_{i} m p^{s+1}\right)\right)} \\
& =\left(p^{m p^{s}}(-1)^{m p^{s+1}}\right)^{(|\mathbf{e}|-|\mathbf{f}|)} \frac{\prod_{i=1}^{q_{1}}\left(p^{e_{i} c}(-1)^{1+e_{i} c p} \Gamma_{p}\left(1+e_{i} c p+e_{i} m p^{s+1}\right)\right)}{\prod_{i=1}^{q_{2}}\left(p^{f_{i} c}(-1)^{1+f_{i} c p} \Gamma_{p}\left(1+f_{i} c p+f_{i} m p^{s+1}\right)\right)} \\
& =\frac{\prod_{i=1}^{q_{1}} p^{e_{i} c}(-1)^{1+e_{i} c p}}{\prod_{i=1}^{q_{2}} p^{f_{i} c}(-1)^{1+f_{i} c p}} \cdot \frac{\prod_{i=1}^{q_{1}} \Gamma_{p}\left(1+e_{i} c p+e_{i} m p^{s+1}\right)}{\prod_{i=1}^{q_{2}} \Gamma_{p}\left(1+f_{i} c p+f_{i} m p^{s+1}\right)} .
\end{aligned}
$$


D'après le point $(i i)$ du lemme 11 , pour tout $n \in \mathbb{N}$, on a $\Gamma_{p}\left(1+n c p+n m p^{s+1}\right) \equiv \Gamma_{p}(1+n c p)$ $\bmod p^{s+1}$. On obtient donc

$$
\frac{\prod_{i=1}^{q_{1}} \Gamma_{p}\left(1+e_{i} c p+e_{i} m p^{s+1}\right)}{\prod_{i=1}^{q_{2}} \Gamma_{p}\left(1+f_{i} c p+f_{i} m p^{s+1}\right)}=\frac{\prod_{i=1}^{q_{1}}\left(\Gamma_{p}\left(1+e_{i} c p\right)+O\left(p^{s+1}\right)\right)}{\prod_{i=1}^{q_{2}}\left(\Gamma_{p}\left(1+f_{i} c p\right)+O\left(p^{s+1}\right)\right)}
$$

où l'on note $x=O\left(p^{k}\right)$ lorsque $x \in p^{k} \mathbb{Z}_{p}$. De plus, par définition de $\Gamma_{p}$, pour tout $n \in \mathbb{N}$, on a $\Gamma_{p}(1+n c p) \in \mathbb{Z}_{p}^{\times}$. On obtient alors

$$
\frac{\prod_{i=1}^{q_{1}}\left(\Gamma_{p}\left(1+e_{i} c p\right)+O\left(p^{s+1}\right)\right)}{\prod_{i=1}^{q_{2}}\left(\Gamma_{p}\left(1+f_{i} c p\right)+O\left(p^{s+1}\right)\right)}=\frac{\prod_{i=1}^{q_{1}} \Gamma_{p}\left(1+e_{i} c p\right)}{\prod_{i=1}^{q_{2}} \Gamma_{p}\left(1+f_{i} c p\right)}\left(1+O\left(p^{s+1}\right)\right)
$$

et ainsi,

$$
\begin{aligned}
\frac{\mathcal{Q}\left(c p+m p^{s+1}\right)}{\mathcal{Q}\left(c+m p^{s}\right)} & =\frac{\prod_{i=1}^{q_{1}} p^{e_{i} c}(-1)^{1+e_{i} c p}}{\prod_{i=1}^{q_{2}} p^{f_{i} c}(-1)^{1+f_{i} c p}} \cdot \frac{\prod_{i=1}^{q_{1}} \Gamma_{p}\left(1+e_{i} c p\right)}{\prod_{i=1}^{q_{2}} \Gamma_{p}\left(1+f_{i} c p\right)}\left(1+O\left(p^{s+1}\right)\right) \\
& =\frac{\prod_{i=1}^{q_{1}} p^{e_{i} c}}{\prod_{i=1}^{q_{2}} p^{f_{i} c}} \cdot \frac{\prod_{i=1}^{q_{1}} \gamma_{p}\left(1+e_{i} c p\right)}{\prod_{i=1}^{q_{2}} \gamma_{p}\left(1+f_{i} c p\right)}\left(1+O\left(p^{s+1}\right)\right) \\
& =\frac{\mathcal{Q}(c p)}{\mathcal{Q}(c)}\left(1+O\left(p^{s+1}\right)\right) .
\end{aligned}
$$

C'est ce qu'il fallait démontrer.

4.4.2. Lorsque $v_{p}(m) \geq \lambda_{p}$. Le but de cette partie est de démontrer le fait suivant.

Soit $p$ un nombre premier et $\lambda_{p}$ l'unique entier naturel tel que $p^{\lambda_{p}}<M_{(\mathbf{e}, \mathbf{f})} \leq p^{\lambda_{p}+1}$. Pour tout $s \in \mathbb{N}$, tout $v \in\{0, \ldots, p-1\}$, tout $u \in\left\{0, \ldots, p^{s}-1\right\}$ et tout $m \in \mathbb{N}$, on a

$$
\frac{\mathcal{Q}\left(v+u p+m p^{s+\lambda_{p}+1}\right)}{\mathcal{Q}(v+u p)}-\frac{\mathcal{Q}\left(u+m p^{s+\lambda_{p}}\right)}{\mathcal{Q}(u)} \in p^{s+\lambda_{p}+1} \frac{g\left(m p^{\lambda_{p}}\right)}{g(v+u p)} \mathbb{Z}_{p} .
$$

L'équation (4.7) est vérifiée si et seulement si, pour tout $v \in\{0, \ldots, p-1\}$, tout $u \in\left\{0, \ldots, p^{s}-\right.$ $1\}$ et tout $m \in \mathbb{N}$, on a

$$
\left(1-\frac{\mathcal{Q}(v+u p)}{\mathcal{Q}(u)} \frac{\mathcal{Q}\left(u+m p^{s+\lambda_{p}}\right)}{\mathcal{Q}\left(v+u p+m p^{s+\lambda_{p}+1}\right)}\right) \frac{\mathcal{Q}\left(v+u p+m p^{s+\lambda_{p}+1}\right)}{\mathcal{Q}(v+u p)} \in p^{s+\lambda_{p}+1} \frac{g\left(m p^{\lambda_{p}}\right)}{g(v+u p)} \mathbb{Z}_{p} .
$$

Dans la suite, on pose $X_{s}(v, u, m):=\frac{\mathcal{Q}(v+u p)}{\mathcal{Q}(u)} \frac{\mathcal{Q}\left(u+m p^{s+\lambda p}\right)}{\mathcal{Q}\left(v+u p+m p^{s+\lambda}+1\right)}$. Ainsi, pour démontrer (4.7), il nous suffit de montrer que

$$
\left(X_{s}(v, u, m)-1\right) \frac{\mathcal{Q}\left(v+u p+m p^{s+\lambda_{p}+1}\right)}{g\left(m p^{\lambda_{p}}\right)} \in p^{s+\lambda_{p}+1} \frac{\mathcal{Q}(v+u p)}{g(v+u p)} \mathbb{Z}_{p} .
$$

Afin d'estimer la valuation de $X_{s}(v, u, m)-1$, posons, pour tout $v \in\{0, \ldots, p-1\}$, tout $u \in$ $\left\{0, \ldots, p^{s}-1\right\}$ et tout $s$ et $m$ dans $\mathbb{N}$,

$$
Y_{s}(v, u, m):=\frac{\prod_{i=1}^{q_{2}} \prod_{j=1}^{\left\lfloor f_{i} v / p\right\rfloor}\left(1+\frac{f_{i} m p^{s+\lambda_{p}}}{f_{i} u+j}\right)}{\prod_{i=1}^{q_{1}} \prod_{j=1}^{\left\lfloor e_{i} v / p\right\rfloor}\left(1+\frac{e_{i} m p^{s+\lambda_{p}}}{e_{i} u+j}\right)} .
$$

Pour $s$ et $m$ dans $\mathbb{N}$ et $a \in\left\{0, \ldots, p^{s}-1\right\}$, on note $\eta_{s}(a, m):=\sum_{\ell=s+1}^{\infty} \Delta\left(\left\{\frac{a+m p^{s}}{p^{\ell}}\right\}\right)$. On va énoncer un certain nombre de lemmes, que l'on démontre dans la partie 4.4.3.

Lemme 12. Pour tout $v \in\{0, \ldots, p-1\}$, tout $u \in\left\{0, \ldots, p^{s}-1\right\}$ et tout $s$ et $m$ dans $\mathbb{N}$, on a $X_{s}(v, u, m) \in Y_{s}(v, u, m)\left(1+p^{s+\lambda_{p}+1} \mathbb{Z}_{p}\right)$ et

$$
v_{p}\left(Y_{s}(v, u, m)\right)=\left(\eta_{s+\lambda_{p}+1}(v+u p, 0)-\eta_{s+\lambda_{p}}(u, 0)\right)-\left(\eta_{s+\lambda_{p}+1}(v+u p, m)-\eta_{s+\lambda_{p}}(u, m)\right) .
$$


Lemme 13. Soit $s \in \mathbb{N}, v \in\{0, \ldots, p-1\}$ et $u \in\left\{0, \ldots, p^{s}-1\right\}$. Si $\left\{(v+u p) / p^{j}\right\}<1 / M_{(\boldsymbol{e}, \boldsymbol{f})}$ pour un $j \in\left\{1, \ldots, s+\lambda_{p}+1\right\}$, alors $Y_{s}(v, u, m) \in 1+p^{s+\lambda_{p}-j+2} \mathbb{Z}_{p}$.

Lemme 14. Pour tout $s \in \mathbb{N}$, tout $a \in\left\{0, \ldots, p^{s+1}-1\right\}$ et tout $m \in \mathbb{N}$, on a

$$
\eta_{s+\lambda_{p}+1}(a, m) \geq \mu\left(m p^{\lambda_{p}}\right)
$$

et

$$
v_{p}\left(\frac{\mathcal{Q}\left(a+m p^{s+\lambda_{p}+1}\right)}{g\left(m p^{\lambda_{p}}\right)}\right) \geq \sum_{\ell=1}^{s+\lambda_{p}+1} \Delta\left(\left\{\frac{a}{p^{\ell}}\right\}\right)
$$

Lemme 15. Pour tout $s \in \mathbb{N}$ et tout $a \in\left\{0, \ldots, p^{s+1}-1\right\}$, on $a: \eta_{s+\lambda_{p}+1}(a, 0)=0$,

$$
v_{p}(Q(a))=\sum_{\ell=1}^{s+\lambda_{p}+1} \Delta\left(\left\{\frac{a}{p^{\ell}}\right\}\right) \quad \text { et } \quad v_{p}(g(a))=\sum_{\ell=1}^{s+\lambda_{p}+1} 1_{\left[1 / M_{(e, f)}, 1[\right.}\left(\left\{\frac{a}{p^{\ell}}\right\}\right) .
$$

Remarque. Le lemme 15 repose essentiellement sur l'inégalité $M_{(\mathbf{e}, \mathbf{f})} \leq p^{\lambda_{p}+1}$.

Afin de montrer (4.9), on va maintenant différencier deux cas.

- Cas 1: Supposons qu'il existe $j \in\left\{1, \ldots, s+\lambda_{p}+1\right\}$ tel que

$$
\left\{\frac{v+u p}{p^{j}}\right\}<\frac{1}{M_{(\mathbf{e}, \mathbf{f})}}
$$

Soit $j_{0}$ le plus petit des $j \in\left\{1, \ldots, s+\lambda_{p}+1\right\}$ vérifiant (4.12). D'après le lemme 13 appliqué en $j_{0}$, on obtient $Y_{s}(v, u, m) \in 1+p^{s+\lambda_{p}-j_{0}+2} \mathbb{Z}_{p}$ et donc, d'après le lemme 12, $v_{p}\left(X_{s}(v, u, m)-1\right) \geq$ $s+\lambda_{p}-j_{0}+2$.

Montrons qu'on a $v_{p}(g(v+u p)) \geq j_{0}-1$. Si $j_{0}=1$, c'est évident. Si $j_{0} \geq 2$, alors, pour tout $\ell \in$ $\left\{1, \ldots, j_{0}-1\right\}$, on a $\left\{(v+u p) / p^{\ell}\right\} \geq 1 / M_{(\mathbf{e}, \mathbf{f})}$ et donc $v_{p}(g(v+u p))=\sum_{\ell=1}^{\infty} \mathbf{1}_{\left[1 / M_{(\mathbf{e}, \mathbf{f})}, 1[\right.}\left(\left\{\frac{v+u p}{p^{\ell}}\right\}\right) \geq$ $j_{0}-1$. D'après (4.11), on obtient

$$
v_{p}\left(\left(X_{s}(v, u, m)-1\right) \frac{\mathcal{Q}\left(v+u p+m p^{s+\lambda_{p}+1}\right)}{g\left(m p^{\lambda_{p}}\right)}\right) \geq v_{p}\left(X_{s}(v, u, m)-1\right)+\sum_{\ell=1}^{s+\lambda_{p}+1} \Delta\left(\left\{\frac{v+u p}{p^{\ell}}\right\}\right) .
$$

D'où

$$
\begin{aligned}
& v_{p}\left(\left(X_{s}(v, u, m)-1\right) \frac{\mathcal{Q}\left(v+u p+m p^{s+\lambda_{p}+1}\right)}{g\left(m p^{\lambda_{p}}\right)}\right) \\
& \quad \geq v_{p}\left(X_{s}(v, u, m)-1\right)+v_{p}(g(v+u p))+\left(\sum_{\ell=1}^{s+\lambda_{p}+1} \Delta\left(\left\{\frac{v+u p}{p^{\ell}}\right\}\right)-v_{p}(g(v+u p))\right) \\
& \quad \geq\left(s+\lambda_{p}-j_{0}+2\right)+j_{0}-1+v_{p}\left(\frac{\mathcal{Q}(v+u p)}{g(v+u p)}\right) \\
& \quad \geq s+\lambda_{p}+1+v_{p}\left(\frac{\mathcal{Q}(v+u p)}{g(v+u p)}\right),
\end{aligned}
$$

où l'on a utilisé l'identité $v_{p}(\mathcal{Q}(v+u p))=\sum_{\ell=1}^{s+\lambda_{p}+1} \Delta\left(\left\{\frac{v+u p}{p^{\ell}}\right\}\right)$ du lemme 15 dans (4.13). On a donc bien (4.9) dans ce cas.

- Cas 2 : Supposons que, pour tout $j \in\left\{1, \ldots, s+\lambda_{p}+1\right\}$, on ait $\left\{(v+u p) / p^{j}\right\} \geq 1 / M_{(\mathbf{e}, \mathbf{f})}$. 
Ainsi, on a $v_{p}(g(v+u p))=\sum_{\ell=1}^{\infty} \mathbf{1}_{\left[1 / M_{(\mathbf{e}, \mathbf{f}), 1}[\right.}\left(\left\{(v+u p) / p^{\ell}\right\}\right) \geq s+\lambda_{p}+1$.

Si $v_{p}\left(Y_{s}(v, u, m)\right) \geq 0$, alors, d'après le lemme [12, $v_{p}\left(X_{s}(v, u, m)-1\right) \geq 0$ et, d'après (4.11) et le lemme 15, on a

$$
\begin{aligned}
v_{p}\left(\frac{\mathcal{Q}\left(v+u p+m p^{s+\lambda_{p}+1}\right)}{g\left(m p^{\lambda_{p}}\right)}\right) & \geq \sum_{\ell=1}^{s+\lambda_{p}+1} \Delta\left(\left\{\frac{v+u p}{p^{\ell}}\right\}\right)=v_{p}(g(v+u p))+v_{p}\left(\frac{\mathcal{Q}(v+u p)}{g(v+u p)}\right) \\
& \geq s+\lambda_{p}+1+v_{p}\left(\frac{\mathcal{Q}(v+u p)}{g(v+u p)}\right) .
\end{aligned}
$$

On a donc bien (4.9).

Supposons maintenant que $v_{p}\left(Y_{s}(v, u, m)\right)<0$. Dans ce cas, d'après le lemme 12, on a

$$
\begin{aligned}
v_{p}\left(X_{s}(v, u, m)-1\right) & =v_{p}\left(Y_{s}(v, u, m)\right) \\
& =\left(\eta_{s+\lambda_{p}+1}(v+u p, 0)-\eta_{s+\lambda_{p}}(u, 0)\right)-\left(\eta_{s+\lambda_{p}+1}(v+u p, m)-\eta_{s+\lambda_{p}}(u, m)\right) .
\end{aligned}
$$

D'après le lemme 15, on a $\eta_{s+\lambda_{p}+1}(v+u p, 0)=0$ et $\eta_{s+\lambda_{p}}(u, 0)=0$ et donc

$$
v_{p}\left(X_{s}(v, u, m)-1\right)=\eta_{s+\lambda_{p}}(u, m)-\eta_{s+\lambda_{p}+1}(v+u p, m)
$$

De plus,

$$
\begin{aligned}
v_{p}\left(\mathcal{Q}\left(v+u p+m p^{s+\lambda_{p}+1}\right)\right) & =\sum_{\ell=1}^{\infty} \Delta\left(\left\{\frac{v+u p+m p^{s+\lambda_{p}+1}}{p^{\ell}}\right\}\right) \\
& =\sum_{\ell=1}^{s+\lambda_{p}+1} \Delta\left(\left\{\frac{v+u p}{p^{\ell}}\right\}\right)+\sum_{\ell=s+\lambda_{p}+2}^{\infty} \Delta\left(\left\{\frac{v+u p+m p^{s+\lambda_{p}+1}}{p^{\ell}}\right\}\right) \\
& =\sum_{\ell=1}^{s+\lambda_{p}+1} \Delta\left(\left\{\frac{v+u p}{p^{\ell}}\right\}\right)+\eta_{s+\lambda_{p}+1}(v+u p, m) .
\end{aligned}
$$

Ainsi, on obtient

$$
\begin{aligned}
& v_{p}\left(\left(X_{s}(v, u, m)-1\right) \frac{\mathcal{Q}\left(v+u p+m p^{s+\lambda_{p}+1}\right)}{g\left(m p^{\lambda_{p}}\right)}\right) \\
& =\eta_{s+\lambda_{p}}(u, m)-\eta_{s+\lambda_{p}+1}(v+u p, m)+\sum_{\ell=1}^{s+\lambda_{p}+1} \Delta\left(\left\{\frac{v+u p}{p^{\ell}}\right\}\right)+\eta_{s+\lambda_{p}+1}(v+u p, m)-\mu\left(m p^{\lambda_{p}}\right) \\
& =\sum_{\ell=1}^{s+\lambda_{p}+1} \Delta\left(\left\{\frac{v+u p}{p^{\ell}}\right\}\right)+\eta_{s+\lambda_{p}}(u, m)-\mu\left(m p^{\lambda_{p}}\right) \\
& =v_{p}(g(v+u p))+v_{p}\left(\frac{\mathcal{Q}(v+u p)}{g(v+u p)}\right)+\eta_{s+\lambda_{p}}(u, m)-\mu\left(m p^{\lambda_{p}}\right) \\
& \geq s+\lambda_{p}+1+v_{p}\left(\frac{\mathcal{Q}(v+u p)}{g(v+u p)}\right)+\eta_{s+\lambda_{p}}(u, m)-\mu\left(m p^{\lambda_{p}}\right) .
\end{aligned}
$$

Si $s=0$, alors on a $u=0$ et $\eta_{\lambda_{p}}(0, m)=\sum_{\ell=\lambda_{p}+1}^{\infty} \Delta\left(\left\{\frac{m p^{\lambda p}}{p^{\ell}}\right\}\right) \geq \sum_{\ell=\lambda_{p}+1}^{\infty} \mathbf{1}_{\left[1 / M_{(\mathbf{e}, \mathbf{f})}, 1[\right.}\left(\left\{\frac{m p^{\lambda_{p}}}{p^{\ell}}\right\}\right)=$ $\mu\left(m p^{\lambda_{p}}\right)$ et on a bien (4.9). En revanche, si $s \geq 1$ alors, en utilisant le lemme 14 avec $s-1$ à la place de $s$ et $a=u$, on obtient $\eta_{s+\lambda_{p}}(u, m) \geq \mu\left(m p^{\lambda_{p}}\right)$, ce qui donne bien (4.9). Ceci achève la preuve de l'équation (4.7), modulo celles des divers lemmes. 


\subsubsection{Démonstration des lemmes 12, 13, 14 et 15.}

Démonstration du lemme 12. On veut montrer que $X_{s}(v, u, m) \in Y_{s}(v, u, m)\left(1+p^{s+\lambda_{p}+1} \mathbb{Z}_{p}\right)$.

On a

$$
X_{s}(v, u, m)=\frac{\mathcal{Q}(v+u p)}{\mathcal{Q}(u p)} \frac{\mathcal{Q}\left(u p+m p^{s+\lambda_{p}+1}\right)}{\mathcal{Q}\left(v+u p+m p^{s+\lambda_{p}+1}\right)} \cdot \frac{\mathcal{Q}(u p)}{\mathcal{Q}(u)} \frac{\mathcal{Q}\left(u+m p^{s+\lambda_{p}}\right)}{\mathcal{Q}\left(u p+m p^{s+\lambda_{p}+1}\right)} .
$$

En appliquant le lemme 10 avec $c=u$ et $s+\lambda_{p}$ pour $s$ au terme tout à droite de (4.14), on obtient

$$
X_{s}(v, u, m) \in \frac{\mathcal{Q}(v+u p)}{\mathcal{Q}(u p)} \frac{\mathcal{Q}\left(u p+m p^{s+\lambda_{p}+1}\right)}{\mathcal{Q}\left(v+u p+m p^{s+\lambda_{p}+1}\right)}\left(1+p^{s+\lambda_{p}+1} \mathbb{Z}_{p}\right) .
$$

De plus, on a

$$
\begin{aligned}
\frac{\mathcal{Q}(v+u p)}{\mathcal{Q}(u p)} \cdot \frac{\mathcal{Q}\left(u p+m p^{s+\lambda_{p}+1}\right)}{\mathcal{Q}\left(v+u p+m p^{s+\lambda_{p}+1}\right)}= & \frac{\left(\prod_{i=1}^{q_{1}} \prod_{k=1}^{e_{i} v}\left(e_{i} u p+k\right)\right)\left(\prod_{i=1}^{q_{2}} \prod_{k=1}^{f_{i} v}\left(f_{i}\left(u p+m p^{s+\lambda_{p}+1}\right)+k\right)\right)}{\left(\prod_{i=1}^{q_{2}} \prod_{k=1}^{f_{i} v}\left(f_{i} u p+k\right)\right)\left(\prod_{i=1}^{q_{1}} \prod_{k=1}^{e_{i} v}\left(e_{i}\left(u p+m p^{s+\lambda_{p}+1}\right)+k\right)\right)} \\
& =\frac{\prod_{i=1}^{q_{2}} \prod_{k=1}^{f_{i} v}\left(1+\frac{f_{i} m p^{s+\lambda_{p}+1}}{f_{i} u p+k}\right)}{\prod_{i=1}^{q_{1}} \prod_{k=1}^{e_{i} v}\left(1+\frac{e_{i} m p^{s+\lambda_{p}+1}}{e_{i} u p+k}\right)} .
\end{aligned}
$$

Si $d \in\left\{e_{1}, \ldots, e_{q_{1}}, f_{1}, \ldots, f_{q_{2}}\right\}$ et $k \in\{1, \ldots, d v\}$, alors $d u p+k$ est divisible par $p$ si et seulement s'il existe $j \in\{1, \ldots,\lfloor d v / p\rfloor\}$ tel que $k=j p$. On a donc

$$
\prod_{k=1}^{d v}\left(1+\frac{d m p^{s+\lambda_{p}+1}}{d u p+k}\right)=\prod_{j=1}^{\lfloor d v / p\rfloor}\left(1+\frac{d m p^{s+\lambda_{p}}}{d u+j}\right)\left(1+O\left(p^{s+\lambda_{p}+1}\right)\right) .
$$

D'où

$$
\begin{aligned}
\frac{\mathcal{Q}(v+u p)}{\mathcal{Q}(u p)} \cdot \frac{\mathcal{Q}\left(u p+m p^{s+\lambda_{p}+1}\right)}{\mathcal{Q}\left(v+u p+m p^{s+\lambda_{p}+1}\right)} & =\frac{\prod_{i=1}^{q_{2}} \prod_{j=1}^{\left\lfloor f_{i} v / p\right\rfloor}\left(1+\frac{f_{i} m p^{s+\lambda_{p}}}{f_{i} u+j}\right)}{\prod_{i=1}^{q_{1}} \prod_{j=1}^{\left\lfloor e_{i} v / p\right\rfloor}\left(1+\frac{e_{i} m p^{s+\lambda_{p}}}{e_{i} u+j}\right)}\left(1+O\left(p^{s+\lambda_{p}+1}\right)\right) \\
& =Y_{s}(v, u, m)\left(1+O\left(p^{s+\lambda_{p}+1}\right)\right)
\end{aligned}
$$

et donc $X_{s}(v, u, m) \in Y_{s}(v, u, m)\left(1+p^{s+\lambda_{p}+1} \mathbb{Z}_{p}\right)$, comme voulu.

On va maintenant montrer qu'on a bien aussi

$$
v_{p}\left(Y_{s}(v, u, m)\right)=\eta_{s+\lambda_{p}+1}(v+u p, 0)-\eta_{s+\lambda_{p}}(u, 0)-\left(\eta_{s+\lambda_{p}+1}(v+u p, m)-\eta_{s+\lambda_{p}}(u, m)\right) .
$$

On a vu ci-dessus que $v_{p}\left(Y_{s}(v, u, m)\right)=v_{p}\left(X_{s}(v, u, m)\right)$. Or, d'après (4.15), on a aussi

$$
\begin{aligned}
v_{p}\left(X_{s}(v, u, m)\right) & =v_{p}\left(\frac{\mathcal{Q}(v+u p)}{\mathcal{Q}(u p)} \cdot \frac{\mathcal{Q}\left(u p+m p^{s+\lambda_{p}+1}\right)}{\mathcal{Q}\left(v+u p+m p^{s+\lambda_{p}+1}\right)}\right) \\
& =v_{p}(\mathcal{Q}(v+u p))-v_{p}(\mathcal{Q}(u p))+v_{p}\left(\mathcal{Q}\left(u p+m p^{s+\lambda_{p}+1}\right)\right)-v_{p}\left(\mathcal{Q}\left(v+u p+m p^{s+\lambda_{p}+1}\right)\right) \\
& =\sum_{\ell=1}^{\infty} \Delta\left(\left\{\frac{v+u p}{p^{\ell}}\right\}\right)-\sum_{\ell=1}^{\infty} \Delta\left(\left\{\frac{u p}{p^{\ell}}\right\}\right)+\sum_{\ell=1}^{\infty} \Delta\left(\left\{\frac{\left.u p+m p^{s+\lambda_{p}+1}\right)}{p^{\ell}}\right\}\right) \\
& -\sum_{\ell=1}^{\infty} \Delta\left(\left\{\frac{v+u p+m p^{s+\lambda_{p}+1}}{p^{\ell}}\right\}\right) .
\end{aligned}
$$


On a

$$
\begin{gathered}
\sum_{\ell=1}^{\infty} \Delta\left(\left\{\frac{v+u p}{p^{\ell}}\right\}\right)-\sum_{\ell=1}^{\infty} \Delta\left(\left\{\frac{v+u p+m p^{s+\lambda_{p}+1}}{p^{\ell}}\right\}\right) \\
=\sum_{\ell=1}^{\infty} \Delta\left(\left\{\frac{v+u p}{p^{\ell}}\right\}\right)-\sum_{\ell=1}^{s+\lambda_{p}+1} \Delta\left(\left\{\frac{v+u p}{p^{\ell}}\right\}\right)-\sum_{\ell=s+\lambda_{p}+2}^{\infty} \Delta\left(\left\{\frac{v+u p+m p^{s+\lambda_{p}+1}}{p^{\ell}}\right\}\right) \\
=\sum_{\ell=s+\lambda_{p}+2}^{\infty} \Delta\left(\left\{\frac{v+u p}{p^{\ell}}\right\}\right)-\sum_{\ell=s+\lambda_{p}+2}^{\infty} \Delta\left(\left\{\frac{v+u p+m p^{s+\lambda_{p}+1}}{p^{\ell}}\right\}\right) \\
=\eta_{s+\lambda_{p}+1}(v+u p, 0)-\eta_{s+\lambda_{p}+1}(v+u p, m),
\end{gathered}
$$

et

$$
\begin{aligned}
& \sum_{\ell=1}^{\infty} \Delta\left(\left\{\frac{u p}{p^{\ell}}\right\}\right)-\sum_{\ell=1}^{\infty} \Delta\left(\left\{\frac{u p+m p^{s+\lambda_{p}+1}}{p^{\ell}}\right\}\right) \\
& =\sum_{\ell=s+\lambda_{p}+2}^{\infty} \Delta\left(\left\{\frac{u p}{p^{\ell}}\right\}\right)-\sum_{\ell=s+\lambda_{p}+2}^{\infty} \Delta\left(\left\{\frac{u p+m p^{s+\lambda_{p}+1}}{p^{\ell}}\right\}\right) \\
& =\sum_{\ell=s+\lambda_{p}+1}^{\infty} \Delta\left(\left\{\frac{u}{p^{\ell}}\right\}\right)-\sum_{\ell=s+\lambda_{p}+1}^{\infty} \Delta\left(\left\{\frac{u+m p^{s+\lambda_{p}}}{p^{\ell}}\right\}\right)=\eta_{s+\lambda_{p}}(u, 0)-\eta_{s+\lambda_{p}}(u, m) .
\end{aligned}
$$

Donc, $v_{p}\left(Y_{s}(v, u, m)\right)=\eta_{s+\lambda_{p}+1}(v+u p, 0)-\eta_{s+\lambda_{p}}(u, 0)-\left(\eta_{s+\lambda_{p}+1}(v+u p, m)-\eta_{s+\lambda_{p}}(u, m)\right)$, ce qui achève la preuve du lemme.

Démonstration du lemme 13. Soit $s \in \mathbb{N}, v \in\{0, \ldots, p-1\}$ et $u \in\left\{0, \ldots, p^{s}-1\right\}$. Soit $\sum_{k=0}^{\infty} u_{k} p^{k}$ le développement $p$-adique de $u$ et $L \in\left\{e_{1}, \ldots, e_{q_{1}}, f_{1}, \ldots, f_{q_{2}}\right\}$. On définit $s+\lambda_{p}+1$ entiers naturels par les relations $b_{L, 0}:=\lfloor L v / p\rfloor$ et $b_{L, k+1}:=\left\lfloor\left(L u_{k}+b_{L, k}\right) / p\right\rfloor$ pour $k \in\left\{0, \ldots, s+\lambda_{p}-1\right\}$. On note, pour tout $x \in \mathbb{R},\lceil x\rceil$ l'entier immédiatement supérieur à $x$ et on définit $s+\lambda_{p}+1$ entiers naturels par les relations $a_{L, 0}:=1$ et $a_{L, k+1}:=\left\lceil\left(L u_{k}+a_{L, k}\right) / p\right\rceil$. Dans un premier temps, nous allons montrer par récurrence sur $r$ que l'assertion $\mathcal{A}_{r}$ :

$$
\prod_{n=1}^{\lfloor L v / p\rfloor}\left(1+\frac{L m p^{s+\lambda_{p}}}{L u+n}\right)=\prod_{n=a_{L, r}}^{b_{L, r}}\left(1+\frac{L m p^{s+\lambda_{p}-r}}{L \sum_{k=r}^{\infty} u_{k} p^{k-r}+n}\right)\left(1+O\left(p^{s+\lambda_{p}-r+1}\right)\right)
$$

est vraie pour tout $r \in\left\{0, \ldots, s+\lambda_{p}\right\}$.

On a $b_{L, 0}=\lfloor L v / p\rfloor$ et $a_{L, 0}=1$, donc $\mathcal{A}_{0}$ est vraie.

Soit $r \geq 0$. Supposons que $\mathcal{A}_{r}$ est vraie et montrons $\mathcal{A}_{r+1}$. Si $n \in\left\{a_{L, r}, \ldots, b_{L, r}\right\}$, alors $p$ divise $L \sum_{k=r}^{\infty} u_{k} p^{k-r}+n$ si et seulement si $p$ divise $L u_{r}+n$, i.e. si et seulement s'il existe $i \in$ $\left\{\left\lceil\left(L u_{r}+a_{L, r}\right) / p\right\rceil, \ldots,\left\lfloor\left(L u_{r}+b_{L, r}\right) / p\right\rfloor\right\}$ tel que $L u_{r}+n=i p$. On obtient donc

$$
\begin{aligned}
\prod_{n=a_{L, r}}^{b_{L, r}}\left(1+\frac{L m p^{s+\lambda_{p}-r}}{L \sum_{k=r}^{\infty} u_{k} p^{k-r}+n}\right) & =\prod_{i=a_{L, r+1}}^{b_{L, r+1}}\left(1+\frac{L m p^{s+\lambda_{p}-r}}{L \sum_{k=r+1}^{\infty} u_{k} p^{k-r}+i p}\right)\left(1+O\left(p^{s+\lambda_{p}-r}\right)\right) \\
& =\prod_{i=a_{L, r+1}}^{b_{L, r+1}}\left(1+\frac{L m p^{s+\lambda_{p}-r-1}}{L \sum_{k=r+1}^{\infty} u_{k} p^{k-r-1}+i}\right)\left(1+O\left(p^{s+\lambda_{p}-r}\right)\right) .
\end{aligned}
$$

D'après $\mathcal{A}_{r}$ et (4.16), on a bien $\mathcal{A}_{r+1}$, ce qui achève la récurrence sur $r$. 
Soit $L \in\left\{e_{1}, \ldots, e_{q_{1}}, f_{1}, \ldots, f_{q_{2}}\right\}$. Nous allons montrer par récurrence sur $k$ que l'assertion $\mathcal{B}_{k}:$ $a_{L, k} \geq 1$ et $b_{L, k} \leq\left\lfloor L\left\{(v+u p) / p^{k+1}\right\}\right\rfloor$ est vraie pour tout $k \in\left\{0, \ldots, s+\lambda_{p}\right\}$.

On a $a_{L, 0}=1$ et $b_{L, 0}=\lfloor L v / p\rfloor=\lfloor L\{(v+u p) / p\}\rfloor$, donc $\mathcal{B}_{0}$ est vraie.

Soit $k \geq 0$. Supposons que $\mathcal{B}_{k}$ est vraie et montrons $\mathcal{B}_{k+1}$. On a $a_{L, k+1}=\left\lceil\left(L u_{k}+a_{L, k}\right) / p\right\rceil$ et $b_{L, k+1}=\left\lfloor\left(L u_{k}+b_{L, k}\right) / p\right\rfloor$, donc $a_{L, k+1} \geq\left\lceil\left(L u_{k}+1\right) / p\right\rceil \geq 1$ et

$$
b_{L, k+1} \leq\left\lfloor\frac{L u_{k}}{p}+\frac{L}{p}\left\{\frac{v+u p}{p^{k+1}}\right\}\right\rfloor=\left\lfloor L\left(\frac{u_{k} p^{k+1}}{p^{k+2}}+\frac{v+p \sum_{i=0}^{k-1} u_{i} p^{i}}{p^{k+2}}\right)\right\rfloor=\left\lfloor L\left\{\frac{v+u p}{p^{k+2}}\right\}\right\rfloor,
$$

ce qui achève la récurrence sur $k$.

Soit $j \in\left\{1, \ldots, s+\lambda_{p}+1\right\}$ tel que $\left\{(v+u p) / p^{j}\right\}<1 / M_{(\mathbf{e}, \mathbf{f})}$. Pour tout $L \in\left\{e_{1}, \ldots, e_{q_{1}}, f_{1}, \ldots, f_{q_{2}}\right\}$, on obtient, via $\mathcal{B}_{j-1}$, que $a_{L, j-1} \geq 1$ et $b_{L, j-1} \leq\left\lfloor L\left\{(v+u p) / p^{j}\right\}\right\rfloor \leq\left\lfloor M_{(\mathbf{e}, \mathbf{f})}\left\{(v+u p) / p^{j}\right\}\right\rfloor=0$. Ainsi, d'après $\mathcal{A}_{j-1}$, on a

$$
\prod_{n=1}^{\lfloor L v / p\rfloor}\left(1+\frac{L m p^{s+\lambda_{p}}}{L u+n}\right)=1+O\left(p^{s+\lambda_{p}-j+2}\right)
$$

et donc

$$
Y_{s}(v, u, m)=\frac{\prod_{i=1}^{q_{2}} \prod_{n=1}^{\left\lfloor f_{i} v / p\right\rfloor}\left(1+\frac{f_{i} m p^{s+\lambda_{p}}}{f_{i} u+n}\right)}{\prod_{i=1}^{q_{1}} \prod_{n=1}^{\left\lfloor e_{i} v / p\right\rfloor}\left(1+\frac{e_{i} m p^{s+\lambda_{p}}}{e_{i} u+n}\right)}=\frac{1+O\left(p^{s+\lambda_{p}-j+2}\right)}{1+O\left(p^{s+\lambda_{p}-j+2}\right)}=1+O\left(p^{s+\lambda_{p}-j+2}\right),
$$

ce qui achève la preuve du lemme 13 .

Démonstration du lemme 14. Dans un premier temps, nous allons montrer qu'on a bien (4.10). Écrivons $m=\sum_{k=0}^{q} m_{k} p^{k}$, où $m_{k} \in\{0, \ldots, p-1\}$. On a

$$
\begin{aligned}
\eta_{s+\lambda_{p}+1} & (a, m)-\mu\left(m p^{\lambda_{p}}\right) \\
& =\sum_{\ell=s+\lambda_{p}+2}^{\infty} \Delta\left(\left\{\frac{a+m p^{s+\lambda_{p}+1}}{p^{\ell}}\right\}\right)-\sum_{\ell=1}^{\infty} 1_{\left[1 / M_{(\mathbf{e}, \mathbf{f})}, 1[\right.}\left(\left\{\frac{m p^{\lambda_{p}}}{p^{\ell}}\right\}\right) \\
& =\sum_{\ell=s+\lambda_{p}+2}^{\infty}\left(\Delta\left(\left\{\frac{a+m p^{s+\lambda_{p}+1}}{p^{\ell}}\right\}\right)-1_{\left[1 / M_{(\mathbf{e}, \mathbf{f})}, 1[\right.}\left(\left\{\frac{m p^{s+\lambda_{p}+1}}{p^{\ell}}\right\}\right)\right) \\
& =\sum_{\ell=s+\lambda_{p}+2}^{\infty}\left(\Delta\left(\frac{a+\sum_{k=0}^{\ell-s-\lambda_{p}-2} m_{k} p^{k+s+\lambda_{p}+1}}{p^{\ell}}\right)-1_{\left[1 / M_{(\mathbf{e}, \mathbf{f})}, 1[\right.}\left(\frac{\sum_{k=0}^{\ell-s-\lambda_{p}-2} m_{k} p^{k+s+\lambda_{p}+1}}{p^{\ell}}\right)\right) .
\end{aligned}
$$

De plus, pour tout $\ell \geq s+\lambda_{p}+2$, on a

$$
0 \leq \frac{\sum_{k=0}^{\ell-s-\lambda_{p}-2} m_{k} p^{k+s+\lambda_{p}+1}}{p^{\ell}} \leq \frac{a+\sum_{k=0}^{\ell-s-\lambda_{p}-2} m_{k} p^{k+s+\lambda_{p}+1}}{p^{\ell}} \leq \frac{p^{\ell}-1}{p^{\ell}}<1 .
$$

Donc

$$
\begin{aligned}
1_{\left[1 / M_{(\mathbf{e}, \mathbf{f})}, 1[\right.}\left(\frac{\sum_{k=0}^{\ell-s-\lambda_{p}-2} m_{k} p^{k+s+\lambda_{p}+1}}{p^{\ell}}\right)=1 & \Longrightarrow 1>\frac{\sum_{k=0}^{\ell-s-\lambda_{p}-2} m_{k} p^{k+s+\lambda_{p}+1}}{p^{\ell}} \geq \frac{1}{M_{(\mathbf{e}, \mathbf{f})}} \\
& \Longrightarrow 1>\frac{a+\sum_{k=0}^{\ell-s-\lambda_{p}-2} m_{k} p^{k+s+\lambda_{p}+1}}{p^{\ell}} \geq \frac{1}{M_{(\mathbf{e}, \mathbf{f})}} \\
& \Longrightarrow \Delta\left(\frac{a+\sum_{k=0}^{\ell-s-\lambda_{p}-2} m_{k} p^{k+s+\lambda_{p}+1}}{p^{\ell}}\right) \geq 1
\end{aligned}
$$


et donc $\eta_{s+\lambda_{p}+1}(a, m)-\mu\left(m p^{\lambda_{p}}\right) \geq 0$. Ceci termine la preuve de (4.10).

Montrons maintenant (4.11). On a

$$
\begin{aligned}
v_{p}\left(\frac{\mathcal{Q}\left(a+m p^{s+\lambda_{p}+1}\right)}{g\left(m p^{\lambda_{p}}\right)}\right) & =\sum_{\ell=1}^{\infty} \Delta\left(\left\{\frac{a+m p^{s+\lambda_{p}+1}}{p^{\ell}}\right\}\right)-\mu\left(m p^{\lambda_{p}}\right) \\
& =\sum_{\ell=1}^{s+\lambda_{p}+1} \Delta\left(\left\{\frac{a}{p^{\ell}}\right\}\right)+\sum_{\ell=s+\lambda_{p}+2}^{\infty} \Delta\left(\left\{\frac{a+m p^{s+\lambda_{p}+1}}{p^{\ell}}\right\}\right)-\mu\left(m p^{\lambda_{p}}\right) \\
& =\sum_{\ell=1}^{s+\lambda_{p}+1} \Delta\left(\left\{\frac{a}{p^{\ell}}\right\}\right)+\eta_{s+\lambda_{p}+1}(a, m)-\mu\left(m p^{\lambda_{p}}\right) \\
& \geq \sum_{\ell=1}^{s+\lambda_{p}+1} \Delta\left(\left\{\frac{a}{p^{\ell}}\right\}\right) .
\end{aligned}
$$

où dans (4.17), on a utilisé l'inégalité (4.10).

Démonstration du lemme 15. On a $\eta_{s+\lambda_{p}+1}(a, 0)=\sum_{\ell=s+\lambda_{p}+2}^{\infty} \Delta\left(\left\{\frac{a}{p^{\ell}}\right\}\right), v_{p}(Q(a))=\sum_{\ell=1}^{\infty} \Delta\left(\left\{\frac{a}{p^{\ell}}\right\}\right)$ et $v_{p}(g(a))=\sum_{\ell=1}^{\infty} 1_{\left[1 / M_{(\mathbf{e}, \mathbf{f})}, 1[\right.}\left(\left\{\frac{a}{p^{\ell}}\right\}\right)$. Or, si $\ell \geq s+\lambda_{p}+2$, on a $\left\{\frac{a}{p^{\ell}}\right\} \leq \frac{p^{s+1}-1}{p^{\ell}}<\frac{1}{p^{\lambda} p+1} \leq \frac{1}{M_{(\mathbf{e}, \mathbf{f})}}$, $\operatorname{car} M_{(\mathbf{e}, \mathbf{f})} \leq p^{\lambda_{p}+1}$. Donc $\Delta\left(\left\{\frac{a}{p^{\ell}}\right\}\right)=1_{\left[1 / M_{(\mathbf{e}, \mathbf{f})}, 1[\right.}\left(\left\{\frac{a}{p^{\ell}}\right\}\right)=0$. D'où le résultat.

\section{Démonstration des points (ii) Des théorèmes 1 et 2}

On se place sous les hypothèses des thèorèmes 1 et 2, On suppose de plus que $\Delta$ s'annule sur $\left[1 / M_{(\mathbf{e}, \mathbf{f})}, 1[\right.$. Le but de cette partie est de montrer qu'il n'existe qu'un nombre fini de premiers $p$ tels que $q(z) \in z \mathbb{Z}_{p}[[z]]$ et que, pour tout $L \in\left\{1, \ldots, M_{(\mathbf{e}, \mathbf{f})}\right\}$, il n'existe qu'un nombre fini de premiers $p$ tels que $q_{L}(z) \in \mathbb{Z}_{p}[[z]]$. On fixe $L \in\left\{1, \ldots, M_{(\mathbf{e}, \mathbf{f})}\right\}$ dans cette partie.

D'après la partie 3, il suffit de montrer que, pour tout nombre premier $p$ assez grand, il existe $a \in\{0, \ldots, p-1\}$ et $K \in \mathbb{N}$ tels que $\Phi_{p}(a+K p) \notin p \mathbb{Z}_{p}$ et $\Phi_{L, p}(a+K p) \notin p \mathbb{Z}_{p}$. En fait, nous allons montrer que pour tout nombre premier $p$ assez grand, il existe $a \in\{0, \ldots, p-1\}$ tel que $\Phi_{p}(a) \notin p \mathbb{Z}_{p}$ et tel que $\Phi_{L, p}(a) \notin p \mathbb{Z}_{p}$ ou $\Phi_{L, p}(a+p) \notin p \mathbb{Z}_{p}$.

5.1. Démonstration du point $(i i)$ du théorème 1. Nous allons montrer que, pour tout nombre premier $p$ assez grand, il existe $a \in\{0, \ldots, p-1\}$ tel que $\Phi_{p}(a) \notin p \mathbb{Z}_{p}$. Dans ce cas, on a $\Phi_{p}(a)=-p \mathcal{Q}(a)\left(\sum_{i=1}^{q_{1}} e_{i} H_{e_{i} a}-\sum_{i=1}^{q_{2}} f_{i} H_{f_{i} a}\right)$.

Pour tout $d \in \mathbb{N}$, on a $H_{d a}=\sum_{i=1}^{d a} \frac{1}{i} \equiv \sum_{j=1}^{\lfloor d a / p\rfloor} \frac{1}{j p} \bmod \mathbb{Z}_{p}$. Pour tout $x \in[0,1]$, on pose $\Psi(x):=$ $\sum_{i=1}^{q_{1}} \sum_{j=1}^{\left\lfloor e_{i} x\right\rfloor} \frac{e_{i}}{j}-\sum_{i=1}^{q_{2}} \sum_{j=1}^{\left\lfloor f_{i} x\right\rfloor} \frac{f_{i}}{j}$. Ainsi, pour tout $a \in\{0, \ldots, p-1\}$, on a $\Phi_{p}(a) \equiv-\mathcal{Q}(a) \Psi(a / p)$ $\bmod p \mathbb{Z}_{p}$. Il suffit donc de montrer que, pour tout premier $p$ assez grand, il existe $a \in\{0, \ldots, p-1\}$ tel que $v_{p}(\mathcal{Q}(a))=v_{p}(\Psi(a / p))=0$.

Pour tout $d \in \mathbb{N}, d \geq 1$, les sauts de l'application $x \mapsto\lfloor d x\rfloor$ sur $[0,1]$ se font aux abscisses $\frac{1}{d}, \frac{2}{d}, \ldots, \frac{d-1}{d}, 1$. Soit $\mathcal{D}:=\left\{e_{1}, \ldots, e_{q_{1}}, f_{1}, \ldots, f_{q_{2}}\right\}$ et $\gamma_{1}<\cdots<\gamma_{t}=1$ les rationnels qui vérifient $\left\{\gamma_{1}, \ldots, \gamma_{t}\right\}=\bigcup_{d \in \mathcal{D}}\left\{\frac{1}{d}, \frac{2}{d}, \ldots, \frac{d-1}{d}, 1\right\}$. Pour tout $i \in\{1, \ldots, t\}$, on note $m_{i} \in \mathbb{Z}$ l'amplitude du saut de $\Delta$ en $\gamma_{i}$. Pour tout $x \in\left[1 / M_{(\mathbf{e}, \mathbf{f})}, 1\left[\right.\right.$, il existe un $i \in\{1, \ldots, t-1\}$ tel que $x \in\left[\gamma_{i}, \gamma_{i+1}[\right.$ et on a alors $\Psi(x)=\sum_{n=1}^{q_{1}} \sum_{j=1}^{\left\lfloor e_{n} x\right\rfloor} \frac{1}{j / e_{n}}-\sum_{n=1}^{q_{2}} \sum_{j=1}^{\left\lfloor f_{n} x\right\rfloor} \frac{1}{j / f_{n}}=\sum_{k=1}^{i} \frac{m_{k}}{\gamma_{k}}$.

La fonction $\Delta$ prend la valeur 0 sur $\left[1 / M_{(\mathbf{e}, \mathbf{f})}, 1\left[\right.\right.$ donc il existe un $i_{0} \in\{1, \ldots, t-1\}$ tel que $\Delta$ soit nulle sur $\left[\gamma_{i_{0}}, \gamma_{i_{0}+1}\right.$ [. Il existe une constante $\mathcal{P}_{1}$ telle que, pour tout premier $p \geq \mathcal{P}_{1}$, il existe un 
$a_{p} \in\{0, \ldots, p-1\}$ tel que $a_{p} / p \in\left[\gamma_{i_{0}}, \gamma_{i_{0}+1}\right.$ [. Ainsi, pour tout premier $p \geq \mathcal{P}_{1}$, on a $\Delta\left(a_{p} / p\right)=0$ et $\Psi\left(a_{p} / p\right)=\sum_{k=1}^{i_{0}} \frac{m_{k}}{\gamma_{k}}$. Il existe une constante $\mathcal{P}_{2} \geq \mathcal{P}_{1}$ telle que, pour tout premier $p \geq \mathcal{P}_{2}$, on ait $\sum_{k=1}^{i_{0}} \frac{m_{k}}{\gamma_{k}} \in \mathbb{Z}_{p}^{\times} \cup\{0\}$. Le lemme suivant nous permet de conclure que l'on a pas $\sum_{k=1}^{i_{0}} \frac{m_{k}}{\gamma_{k}}=0$.

Lemme 16. Soit $\boldsymbol{e}:=\left(e_{1}, \ldots, e_{q_{1}}\right)$ et $\boldsymbol{f}:=\left(f_{1}, \ldots, f_{q_{2}}\right)$ deux suites d'entiers strictement positifs disjointes. On se place dans les conditions ci-dessus. S'il existe $i_{0} \in\{1, \ldots, t\}$ tel que $\Delta_{(\boldsymbol{e}, \boldsymbol{f})}$ soit positive sur $\left[\gamma_{1}, \gamma_{i_{0}}\left[\right.\right.$ et nulle sur $\left[\gamma_{i_{0}}, \gamma_{i_{0}+1}[\right.$, alors on a

$$
\sum_{k=1}^{i_{0}} \frac{m_{k}}{\gamma_{k}}>0 \text { et } \prod_{k=1}^{i_{0}}\left(1+\frac{1}{\gamma_{k}}\right)^{m_{k}}>1
$$

Remarque. On utilisera l'inégalité $\prod_{k=1}^{i_{0}}\left(1+\frac{1}{\gamma_{k}}\right)^{m_{k}}>1$ dans la démonstration du point $(i i)$ du théorème 2.

D'après ce lemme, pour tout premier $p \geq \max \left(\mathcal{P}_{2}, M_{(\mathbf{e}, \mathbf{f})}\right)$, il existe un $a_{p} \in\{0, \ldots, p-1\}$ tel que $\Delta\left(a_{p} / p\right)=0$ et $\Psi\left(a_{p} / p\right) \in \mathbb{Z}_{p}^{\times}$, car $\Psi\left(a_{p} / p\right)=\sum_{k=1}^{i_{0}} \frac{m_{k}}{\gamma_{k}} \neq 0$. Pour tout $\ell \in \mathbb{N}, \ell \geq 2$, on a $a_{p} / p^{\ell}<$ $1 / p \leq 1 / M_{(\mathbf{e}, \mathbf{f})}$ et donc $\Delta\left(\left\{a_{p} / p^{\ell}\right\}\right)=0$. Ainsi, on obtient bien $v_{p}\left(\mathcal{Q}\left(a_{p}\right)\right)=\sum_{\ell=1}^{\infty} \Delta\left(\left\{a_{p} / p^{\ell}\right\}\right)=0$ et $\Psi\left(a_{p} / p\right) \in \mathbb{Z}_{p}^{\times}$, ce qui termine la démonstration de l'assertion (ii) du théorème 1 .

Démonstration du lemme 16. Les suites e et $\mathbf{f}$ sont disjointes donc $\gamma_{1}=1 / M_{(\mathbf{e}, \mathbf{f})}$ est un saut effectif de $\Delta$. Comme, pour tout $x \in[0,1]$, on a $\Delta(x) \geq 0$, on obtient $\Delta\left(\gamma_{1}\right) \geq 1$, i.e. $m_{1} \geq 1$. Comme $\Delta$ est nulle $\operatorname{sur}\left[\gamma_{i_{0}}, \gamma_{i_{0}+1}\right.$ [, on a $\sum_{k=1}^{i_{0}} m_{k}=0$. Or $m_{1} \geq 1$ donc $i_{0} \geq 2$. On va maintenant montrer par récurrence sur $\ell$ que, pour tout $\ell \in\left\{2, \ldots, i_{0}\right\}$, on a

$$
\sum_{k=1}^{\ell} \frac{m_{k}}{\gamma_{k}}>\frac{1}{\gamma_{\ell}} \sum_{k=1}^{\ell} m_{k} \text { et } \prod_{k=1}^{\ell}\left(1+\frac{1}{\gamma_{k}}\right)^{m_{k}}>\left(1+\frac{1}{\gamma_{\ell}}\right)^{\sum_{k=1}^{\ell} m_{k}} .
$$

On a $\frac{1}{\gamma_{1}}>\cdots>\frac{1}{\gamma_{t}}$ et $m_{1} \geq 1$ donc

$$
\frac{m_{1}}{\gamma_{1}}+\frac{m_{2}}{\gamma_{2}}>\frac{m_{1}}{\gamma_{2}}+\frac{m_{2}}{\gamma_{2}} \text { et }\left(1+\frac{1}{\gamma_{1}}\right)^{m_{1}}\left(1+\frac{1}{\gamma_{2}}\right)^{m_{2}}>\left(1+\frac{1}{\gamma_{2}}\right)^{m_{1}+m_{2}} \text {. }
$$

Ainsi, (5.1) est vraie pour $\ell=2$. Si $i_{0} \geq 3$, soit $\ell \in\left\{2, \ldots, i_{0}-1\right\}$ tel que (5.1) soit vraie. Par hypothèse de récurrence, on a

$$
\sum_{k=1}^{\ell+1} \frac{m_{k}}{\gamma_{k}}>\frac{1}{\gamma_{\ell}} \sum_{k=1}^{\ell} m_{k}+\frac{m_{\ell+1}}{\gamma_{\ell+1}} \text { et } \prod_{k=1}^{\ell+1}\left(1+\frac{1}{\gamma_{k}}\right)^{m_{k}}>\left(1+\frac{1}{\gamma_{\ell}}\right)^{\sum_{k=1}^{\ell} m_{k}}\left(1+\frac{1}{\gamma_{\ell+1}}\right)^{m_{\ell+1}} .
$$

Comme $\Delta$ est positive $\operatorname{sur}\left[\gamma_{1}, \gamma_{i_{0}}\right.$, on obtient $\sum_{k=1}^{\ell} m_{k} \geq 0$ et donc $\frac{1}{\gamma_{\ell}} \sum_{k=1}^{\ell} m_{k} \geq \frac{1}{\gamma_{\ell+1}} \sum_{k=1}^{\ell} m_{k}$ et $\left(1+\frac{1}{\gamma_{\ell}}\right)^{\sum_{k=1}^{\ell} m_{k}} \geq\left(1+\frac{1}{\gamma_{\ell+1}}\right)^{\sum_{k=1}^{\ell} m_{k}}$. Ainsi, on a bien

$$
\sum_{k=1}^{\ell+1} \frac{m_{k}}{\gamma_{k}}>\frac{1}{\gamma_{\ell+1}} \sum_{k=1}^{\ell+1} m_{k} \text { et } \prod_{k=1}^{\ell+1}\left(1+\frac{1}{\gamma_{k}}\right)^{m_{k}}>\left(1+\frac{1}{\gamma_{\ell+1}}\right)^{\sum_{k=1}^{\ell+1} m_{k}},
$$

ce qui achève la récurrence.

En utilisant (5.1) avec $\ell=i_{0}$, on obtient $\sum_{k=1}^{i_{0}} \frac{m_{k}}{\gamma_{k}}>\frac{1}{\gamma_{i_{0}}} \sum_{k=1}^{i_{0}} m_{k}=0$ et $\prod_{k=1}^{i_{0}}\left(1+\frac{1}{\gamma_{k}}\right)^{m_{k}}>$ $\left(1+\frac{1}{\gamma_{i_{0}}}\right)^{\sum_{k=1}^{i_{0}} m_{k}}=1$, ce qui achève la preuve du lemme. 
5.2. Démonstration du point $(i i)$ du théorème 2. On fixe $L \in\left\{1, \ldots, M_{(\mathbf{e}, \mathbf{f})}\right\}$ dans cette partie. Nous allons montrer que, pour tout nombre premier $p$ assez grand, il existe $a \in\{0, \ldots, p-1\}$ tel que $\Phi_{L, p}(a) \notin p \mathbb{Z}_{p}$ ou $\Phi_{L, p}(a+p) \notin p \mathbb{Z}_{p}$. On rappelle qu'on a $\Phi_{L, p}(a)=-p \mathcal{Q}(a) H_{L a}$.

Comme on l'a dit dans la partie 5.1, on a $H_{L a} \equiv \sum_{j=1}^{\lfloor L a / p\rfloor} \frac{1}{j p} \bmod p \mathbb{Z}_{p}$, ce qui donne $\Phi_{L, p}(a) \equiv$ $-\mathcal{Q}(a) H_{\lfloor L a / p\rfloor} \bmod p \mathbb{Z}_{p}$. Pour tout premier $p$ et tout $a \in\{0, \ldots, p-1\}$, on a $H_{\lfloor L a / p\rfloor} \in\left\{0, H_{1}, \ldots, H_{L}\right\}$. Il existe une constante $\mathcal{P}_{1}$ telle que, pour tout premier $p \geq \mathcal{P}_{1}$, on ait $\left\{H_{1}, \ldots, H_{L}\right\} \subset \mathbb{Z}_{p}^{\times}$. D'après la partie 5.1, il existe une constante $\mathcal{P}_{2}>M_{(\mathbf{e}, \mathbf{f})}$ telle que, pour tout premier $p \geq \mathcal{P}_{2}$, il existe $a \in\{0, \ldots, p-1\}$ tel que $v_{p}(\mathcal{Q}(a))=0$. Pour tout premier $p \geq \mathcal{P}_{0}:=\max \left(\mathcal{P}_{1}, \mathcal{P}_{2}\right)$, alors il existe $a_{p} \in\{0, \ldots, p-1\}$ tel que $v_{p}\left(\mathcal{Q}\left(a_{p}\right)\right)=0$ et $H_{\left\lfloor L a_{p} / p\right\rfloor} \in \mathbb{Z}_{p}^{\times} \cup\{0\}$. Ainsi, si $\left\lfloor L a_{p} / p\right\rfloor \geq 1$, alors on a $\Phi_{L, p}\left(a_{p}\right) \notin p \mathbb{Z}_{p}$. On remarque cependant que si $L=1$, alors on a toujours $\left\lfloor 1 \cdot a_{p} / p\right\rfloor=0$ et donc $\Phi_{1, p}\left(a_{p}\right) \in p \mathbb{Z}_{p}$. On fixe un premier $p \geq \mathcal{P}_{0}$ dans la suite.

Nous allons maintenant montrer que si $\left\lfloor L a_{p} / p\right\rfloor=0$, alors on a $\Phi_{L, p}\left(a_{p}+p\right) \notin p \mathbb{Z}_{p}$, ce qui achèvera la preuve du point $(i i)$ du théorème 2 , Si $\left\lfloor L a_{p} / p\right\rfloor=0$, alors on a $-p \mathcal{Q}\left(a_{p}\right) H_{L a_{p}} \in p \mathbb{Z}_{p}$. De plus, on a $H_{L\left(a_{p}+p\right)}=\sum_{j=1}^{L\left(a_{p}+p\right)} \frac{1}{j} \equiv \frac{1}{p} \sum_{i=1}^{\left\lfloor\left(a_{p}+p\right) / p\right\rfloor} \frac{1}{i} \bmod \mathbb{Z}_{p}$ et $\left\lfloor L\left(a_{p}+p\right) / p\right\rfloor=L$ donc $p H_{L\left(a_{p}+p\right)} \equiv H_{L} \bmod p \mathbb{Z}_{p}$. On obtient

$$
\begin{aligned}
\Phi_{L, p}\left(a_{p}+p\right) & =\sum_{j=0}^{1} \mathcal{Q}(1-j) \mathcal{Q}\left(a_{p}+j p\right)\left(H_{L(1-j)}-p H_{L\left(a_{p}+j p\right)}\right) \\
& =\mathcal{Q}(1) \mathcal{Q}\left(a_{p}\right)\left(H_{L}-p H_{L a_{p}}\right)-\mathcal{Q}\left(a_{p}+p\right) p H_{L\left(a_{p}+p\right)} \\
& \equiv \mathcal{Q}(1) \mathcal{Q}\left(a_{p}\right) H_{L}-\mathcal{Q}\left(a_{p}+p\right) H_{L} \quad \bmod p \mathbb{Z}_{p} \\
& \equiv H_{L} \mathcal{Q}\left(a_{p}\right) \mathcal{Q}(1)\left(1-\frac{\mathcal{Q}\left(a_{p}+p\right)}{\mathcal{Q}\left(a_{p}\right) \mathcal{Q}(1)}\right) \quad \bmod p \mathbb{Z}_{p}
\end{aligned}
$$

avec $H_{L}$ et $\mathcal{Q}\left(a_{p}\right)$ dans $\mathbb{Z}_{p}^{\times}$. De plus, on a $p>M_{(\mathbf{e}, \mathbf{f})}$ donc $v_{p}(\mathcal{Q}(1))=\sum_{\ell=1}^{\infty} \Delta\left(\left\{1 / p^{\ell}\right\}\right)=0$ car $\Delta$ est nulle sur $\left[0,1 / M_{(\mathbf{e}, \mathbf{f})}\left[\right.\right.$. Il nous suffit donc de montrer que $1-\frac{\mathcal{Q}\left(a_{p}+p\right)}{\mathcal{Q}\left(a_{p}\right) \mathcal{Q}(1)} \in \mathbb{Z}_{p}^{\times}$. On a

$$
\frac{\mathcal{Q}\left(a_{p}+p\right)}{\mathcal{Q}\left(a_{p}\right)}=\frac{\prod_{i=1}^{q_{1}} \prod_{j=1}^{e_{i} p}\left(e_{i} a_{p}+j\right)}{\prod_{i=1}^{q_{2}} \prod_{j=1}^{f_{i} p}\left(f_{i} a_{p}+j\right)}
$$

Pour tout $d \in\left\{1, \ldots, M_{(\mathbf{e}, \mathbf{f})}\right\}$, on a $\prod_{j=1}^{d p}\left(d a_{p}+j\right)=\prod_{k=0}^{d-1} \prod_{j=1}^{p}\left(d a_{p}+j+k p\right)$. Comme $p>M_{(\mathbf{e}, \mathbf{f})}$, on a $\left\lfloor d a_{p} / p^{2}\right\rfloor=0$ donc il existe $n_{d} \in\{0, \ldots, p-1\}$ tel que $d a_{p}=n_{d}+\left\lfloor d a_{p} / p\right\rfloor p$. Pour tout $k \in\{0, \ldots, d-1\}$, on a alors

$$
\prod_{\substack{j=1 \\ j \neq p-n_{d}}}^{p}\left(d a_{p}+j+k p\right)=\prod_{m=1}^{p-1}(m+O(p))=-1+O(p) \quad \text { et } \quad d a_{p}+p-n_{d}+k p=p\left(\left\lfloor d a_{p} / p\right\rfloor+1+k\right)
$$


ce qui donne $\prod_{j=1}^{d p}\left(d a_{p}+j\right)=(-1+O(p))^{d} p^{d} \prod_{k=0}^{d-1}\left(\left\lfloor d a_{p} / p\right\rfloor+1+k\right)=\left((-1)^{d}+O(p)\right) p^{d} \prod_{k=0}^{d-1}\left(\left\lfloor d a_{p} / p\right\rfloor+\right.$ $1+k)$. Ainsi, on obtient

$$
\begin{aligned}
\frac{\mathcal{Q}\left(a_{p}+p\right)}{\mathcal{Q}\left(a_{p}\right)} & =\frac{\prod_{i=1}^{q_{1}}\left(\left((-1)^{e_{i}}+O(p)\right) p^{e_{i}} \prod_{k=1}^{e_{i}}\left(\left\lfloor e_{i} a_{p} / p\right\rfloor+k\right)\right)}{\prod_{i=1}^{q_{2}}\left(\left((-1)^{f_{i}}+O(p)\right) p^{f_{i}} \prod_{k=1}^{f_{i}}\left(\left\lfloor f_{i} a_{p} / p\right\rfloor+k\right)\right)} \\
& =\left((-1)^{|\mathbf{e}|}+O(p)\right)\left((-1)^{|\mathbf{f}|}+O(p)\right) p^{|\mathbf{e}|-|\mathbf{f}|} \frac{\prod_{i=1}^{q_{1}}\left(\left\lfloor e_{i} a_{p} / p\right\rfloor+e_{i}\right) !}{\prod_{i=1}^{q_{2}}\left(\left\lfloor f_{i} a_{p} / p\right\rfloor+f_{i}\right) !} \cdot \frac{\prod_{i=1}^{q_{2}}\left\lfloor f_{i} a_{p} / p\right\rfloor !}{\prod_{i=1}^{q_{1}}\left\lfloor e_{i} a_{p} / p\right\rfloor !} \\
& =\frac{\prod_{i=1}^{q_{2}}\left\lfloor f_{i} a_{p} / p\right\rfloor !}{\prod_{i=1}^{q_{1}}\left\lfloor e_{i} a_{p} / p\right\rfloor !} \cdot \frac{\prod_{i=1}^{q_{1}} e_{i} !}{\prod_{i=1}^{q_{2}} f_{i} !} \cdot \frac{\prod_{i=1}^{q_{1}} \prod_{k=1}^{\left\lfloor e_{i} a_{p} / p\right\rfloor}\left(e_{i}+k\right)}{\prod_{i=1}^{q_{2}} \prod_{k=1}^{\left\lfloor f_{i} a_{p} / p\right\rfloor}\left(f_{i}+k\right)}(1+O(p)),
\end{aligned}
$$

ce qui donne

$$
\frac{\mathcal{Q}\left(a_{p}+p\right)}{\mathcal{Q}\left(a_{p}\right) \mathcal{Q}(1)}=\frac{\prod_{i=1}^{q_{1}} \prod_{k=1}^{\left.e_{i} a_{p} / p\right\rfloor}\left(\frac{e_{i}}{k}+1\right)}{\prod_{i=1}^{q_{2}} \prod_{k=1}^{\left\lfloor f_{i} a_{p} / p\right\rfloor}\left(\frac{f_{i}}{k}+1\right)}(1+O(p))=\prod_{k=1}^{i_{0}}\left(1+\frac{1}{\gamma_{k}}\right)^{m_{k}}(1+O(p)),
$$

où la dernière égalité a lieu car $a_{p} / p \in\left[\gamma_{i_{0}}, \gamma_{i_{0}+1}\left[\right.\right.$. Le lemme 16 nous dit que $\prod_{k=1}^{i_{0}}\left(1+\frac{1}{\gamma_{k}}\right)^{m_{k}}>1$, on ne peut donc avoir $\prod_{k=1}^{i_{0}}\left(1+\frac{1}{\gamma_{k}}\right)^{m_{k}}=1+O(p)$ que pour un nombre fini de premier $p$, car rappelons que si $x \in \mathbb{Q}$ est dans $p \mathbb{Z}_{p}$ pour une infinité de nombres premiers $p$, alors $x=0$. Ainsi, pour tout premier $p$ assez grand, on a bien $\left\lfloor L a_{p} / p\right\rfloor=0 \Rightarrow \Phi_{L, p}\left(a_{p}+p\right) \notin p \mathbb{Z}_{p}$, ce qui termine la preuve du point $(i i)$ du théorème 2 ,

\section{RÉSUltats HYPERGÉOMÉTRIQUES}

Le but de cette partie est de caractériser les séries hypergéométriques généralisées dont les coefficients peuvent se mettre sous forme factorielle. Cela nous permettra de décrire les sauts de l'application de Landau sur $[0,1]$ et d'en conclure que $\Delta_{(\mathbf{e}, \mathbf{f})}$ est croissante sur $[0,1$ [ si et seulement si l'équation différentielle fuchsienne associée à $F_{(\mathbf{e}, \mathbf{f})}$ a tous ses exposants égaux à 0 à l'origine.

6.1. Séries hypergéométriques définies par des quotients de factorielles. Soit e et $\mathbf{f}$ deux suites d'entiers positifs. La série formelle $F_{(\mathbf{e}, \mathbf{f})}(z)=\sum_{n=0}^{\infty} \mathcal{Q}_{(\mathbf{e}, \mathbf{f})}(n) z^{n}$ est une série hypergéométrique (voir [2, Lemma 4.1, p. 431]). Nous allons caractériser les suites $\left(\alpha_{1}, \ldots, \alpha_{r}\right) \in \mathbb{C}^{r}$ et $\left(\beta_{1}, \ldots, \beta_{s}\right) \in\left(\mathbb{C} \backslash \mathbb{Z}_{\leq 0}\right)^{s}$ telles qu'il existe deux suites d'entiers positifs e et f vérifiant

$$
{ }_{r} F_{s}\left(\begin{array}{c}
\alpha_{1}, \ldots, \alpha_{r} \\
\beta_{1}, \ldots, \beta_{s}
\end{array} ; C z\right):=\sum_{n=0}^{\infty} C^{n} \frac{\left(\alpha_{1}\right)_{n} \cdots\left(\alpha_{r}\right)_{n}}{\left(\beta_{1}\right)_{n} \cdots\left(\beta_{s}\right)_{n}} \frac{z^{n}}{n !}=\sum_{n=0}^{\infty} \frac{\left(e_{1} n\right) ! \cdots\left(e_{q_{1}} n\right) !}{\left(f_{1} n\right) ! \cdots\left(f_{q_{2}} n\right) !} z^{n}=: F_{(\mathbf{e}, \mathbf{f})}(z),
$$

où $(x)_{n}:=x(x+1) \cdots(x+n-1)$ pour $n \geq 1$ et $(x)_{0}=1$ (symbole de Pochhammer). Nous allons avoir besoin du résultat d'unicité suivant.

Proposition 1. Soit $C$ et $C^{\prime}$ deux constantes strictement positives, $\alpha_{1}, \ldots, \alpha_{r}, \alpha_{1}^{\prime}, \ldots, \alpha_{r^{\prime}}^{\prime}, \beta_{1}, \ldots, \beta_{s}$ et $\beta_{1}^{\prime}, \ldots, \beta_{s^{\prime}}^{\prime}$ des complexes où aucun des $\beta_{j}$ ni aucun des $\beta_{j}^{\prime}$ n'est un entier négatif tels que, pour tout $(i, j) \in\{1, \ldots, r\} \times\{1, \ldots, s\}$, on ait $\alpha_{i} \neq \beta_{j}$, et, pour tout $(i, j) \in\left\{1, \ldots, r^{\prime}\right\} \times\left\{1, \ldots, s^{\prime}\right\}$, on ait $\alpha_{i}^{\prime} \neq \beta_{j}^{\prime}$. Si, pour tout $n \in \mathbb{N}$, on a

$$
C^{n} \frac{\left(\alpha_{1}\right)_{n} \cdots\left(\alpha_{r}\right)_{n}}{n !\left(\beta_{1}\right)_{n} \cdots\left(\beta_{s}\right)_{n}}=C^{\prime n} \frac{\left(\alpha_{1}^{\prime}\right)_{n} \cdots\left(\alpha_{r^{\prime}}^{\prime}\right)_{n}}{n !\left(\beta_{1}^{\prime}\right)_{n} \cdots\left(\beta_{s^{\prime}}^{\prime}\right)_{n}}
$$

alors on a $C=C^{\prime}, r=r^{\prime}, s=s^{\prime}$ et il existe une permutation $\sigma \in S_{r}$ et une permutation $\tau \in S_{s}$ telles que, pour tout $i \in\{1, \ldots, r\}$, on ait $\alpha_{i}=\alpha_{\sigma(i)}^{\prime}$ et, pour tout $j \in\{1, \ldots, s\}$, on ait $\beta_{j}=\beta_{\tau(j)}^{\prime}$. 
Démonstration. Soit $i$ dans $\mathbb{N}$, en divisant l'identité (6.2) avec $n=i+1$ par celle avec $n=i$ on obtient, pour tout $i$ dans $\mathbb{N}, i \geq 1, C \frac{\left(\alpha_{1}+i\right) \cdots\left(\alpha_{r}+i\right)}{\left(\beta_{1}+i\right) \cdots\left(\beta_{s}+i\right)}=C^{\prime} \frac{\left(\alpha_{1}^{\prime}+i\right) \cdots\left(\alpha_{r^{\prime}}^{\prime}+i\right)}{\left(\beta_{1}^{\prime}+i\right) \cdots\left(\beta_{s^{\prime}}^{\prime}+i\right)}$. Ainsi, les deux fractions rationnelles $P(X):=C \frac{\left(\alpha_{1}+X\right) \cdots\left(\alpha_{r}+X\right)}{\left(\beta_{1}+X\right) \cdots\left(\beta_{s}+X\right)}$ et $Q(X):=C^{\prime} \frac{\left(\alpha_{1}^{\prime}+X\right) \cdots\left(\alpha_{r^{\prime}}^{\prime}+X\right)}{\left(\beta_{1}^{\prime}+X\right) \cdots\left(\beta_{s^{\prime}}^{\prime}+X\right)}$ sont égales. Elles ont donc les mêmes racines avec mêmes multiplicités et les mêmes pôles avec mêmes ordres. D'où le résultat.

Afin de caractériser la forme des suites $\left(\alpha_{1}, \ldots, \alpha_{r}\right)$ et $\left(\beta_{1}, \ldots, \beta_{s}\right)$ vérifiant (6.1) , on définit un certain type de partition de multi-ensemble.

Définition. Pour $N \in \mathbb{N}, N \geq 1$, on note $R_{N}:=\{w / N: 1 \leq w \leq N,(w, N)=1\}$. On dira qu'un multi-ensemble $\left\{\alpha_{1}, \ldots, \alpha_{r}\right\}$ de réels est $R$-partitionné selon le multi-ensemble $\left\{N_{1}, \ldots, N_{k}\right\}$ s'il existe des entiers strictement positifs $N_{1}, \ldots, N_{k}$ et une partition $E_{1}, \ldots, E_{k}$ de $\{1, \ldots, r\}$ tels que, pour tout $j \in\{1, \ldots, k\}, \operatorname{Card}\left(E_{j}\right)=\varphi\left(N_{j}\right)$ et $\left\{\alpha_{i}: i \in E_{j}\right\}=R_{N_{j}}$.

Par exemple, le multi-ensemble $\{1 / 3,1 / 2,1 / 2,2 / 3\}$ est $R$-partitionné selon le multi-ensemble $\{2,2,3\}$.

Si $N$ est un entier non nul, on note $C_{N}:=N^{\varphi(N)} \prod_{p \mid N} p^{\frac{\varphi(N)}{p-1}} \in \mathbb{N}, C_{N} \geq 1$. Si $\alpha$ est une suite $R$-partitionnée selon $\left(N_{1}, \ldots, N_{k}\right)$, on note $C_{\alpha}:=C_{N_{1}} \cdots C_{N_{k}}$. Si $\alpha$ et $\beta$ sont deux suites $R$ partitionnées, on note $C_{(\alpha, \beta)}:=C_{\alpha} / C_{\beta}$. On note $Q$ l'ensemble des suites de la forme $\mathcal{Q}_{(\mathbf{e}, \mathbf{f})}(n):=$ $\frac{\left(e_{1} n\right) ! \cdots\left(e_{q_{1}} n\right) !}{\left(f_{1} n\right) ! \cdots\left(f_{q_{2}} n\right) !}$, où $q_{1}, q_{2} \in \mathbb{N}, q_{1} q_{2} \neq 0, e_{1}, \ldots, e_{q_{1}}, f_{1}, \ldots, f_{q_{2}} \in \mathbb{N}$. On note $P$ l'ensemble des suites de la forme $\mathcal{P}_{(\alpha, \beta)}(n):=C_{(\alpha, \beta)}^{n} \frac{\left(\alpha_{1}\right)_{n} \cdots\left(\alpha_{r}\right)_{n}}{n !\left(\beta_{1}\right)_{n} \cdots\left(\beta_{s}\right)_{n}}$, où $r, s \in \mathbb{N}$, rs $\neq 0,\left(\alpha_{1}, \ldots, \alpha_{r}\right)$ et $\left(\beta_{1}, \ldots, \beta_{s}\right)$ sont deux suites $R$-partitionnées.

Proposition 2. On a $P=Q$ et, si $C_{(\alpha, \beta)}^{n} \frac{\left(\alpha_{1}\right)_{n} \cdots\left(\alpha_{r}\right)_{n}}{n !\left(\beta_{1}\right)_{n} \cdots\left(\beta_{s}\right)_{n}}=\frac{\left(e_{1} n\right) ! \cdots\left(e_{q_{1}} n\right) !}{\left(f_{1} n\right) ! \cdots\left(f_{q_{2}} n\right) !}$ pour tout $n \geq 0$, alors $C_{(\alpha, \beta)}=\frac{e_{1}^{e_{1} \ldots e_{q_{1}}^{e_{q_{1}}}}}{f_{1}^{f_{1} \ldots f_{q_{2}}^{f}}}$ et $r-s-1=|\boldsymbol{e}|-|\boldsymbol{f}|$.

Cette proposition caractérise complètement les fonctions hypergéométriques dont les coefficients peuvent se mettre sous forme de quotients de factorielles. Pour démontrer la proposition 2, on va utiliser un lemme dû à Zudilin ([13, Lemma 4, p. 609]).

Lemme 17 (Zudilin). Soit $N \geq 2$ un entier. On écrit $N=p_{1}^{a_{1}} p_{2}^{a_{2}} \cdots p_{\ell}^{a_{\ell}}$ sa décomposition en produit de facteurs premiers. Pour tout $n \in \mathbb{N}$, on a alors $C_{N}^{n} \frac{\prod_{\alpha \in R_{N}}(\alpha)_{n}}{(n !) \varphi(N)}=\frac{\left(e_{1} n\right) ! \cdots\left(e_{q_{1}} n\right) !}{\left(f_{1} n\right) ! \cdots\left(f_{q_{2}} n\right) !}$, où

$$
\begin{gathered}
\left\{e_{i}\right\}_{i=1, \ldots, q_{1}}=\left\{N, \frac{N}{p_{j_{1}} p_{j_{2}}}, \frac{N}{p_{j_{1}} p_{j_{2}} p_{j_{3}} p_{j_{4}}}, \ldots\right\}_{1 \leq j_{1}<j_{2}<\ldots \leq \ell}, \\
\left\{f_{j}\right\}_{j=1, \ldots, q_{2}}=\left\{1, \ldots, 1, \frac{N}{p_{j_{1}}}, \frac{N}{p_{j_{1}} p_{j_{2}} p_{j_{3}}}, \ldots\right\}_{1 \leq j_{1}<j_{2}<\ldots \leq \ell}
\end{gathered}
$$

et de plus, $|\boldsymbol{e}|=|\boldsymbol{f}|$.

Démonstration de la proposition [2. Montrons que $P \subset Q$.

Soit $\alpha:=\left(\alpha_{1}, \ldots, \alpha_{r}\right)$ et $\beta:=\left(\beta_{1}, \ldots, \beta_{s}\right)$ deux suites $R$-partitionnées respectivement selon $\left(N_{1}, \ldots, N_{k}\right)$ et $\left(N_{1}^{\prime}, \ldots, N_{k^{\prime}}^{\prime}\right)$. Quitte à réordonner les suites $\left(N_{1}, \ldots, N_{k}\right)$ et $\left(N_{1}^{\prime}, \ldots, N_{k^{\prime}}^{\prime}\right)$, on peut supposer qu'il existe $k_{0} \in\{0, \ldots, k\}$ et $k_{0}^{\prime} \in\left\{0, \ldots, k^{\prime}\right\}$ tels que, pour tout $i \in\left\{1, \ldots, k_{0}\right\}$ et tout $j \in\left\{1, \ldots, k_{0}^{\prime}\right\}$, on ait $N_{i} \geq 2$ et $N_{j}^{\prime} \geq 2$, et, pour tout $i \in\left\{k_{0}+1, \ldots, k\right\}$ et tout $j \in\left\{k_{0}^{\prime}+1, \ldots, k^{\prime}\right\}$, on ait $N_{i}=1$ et $N_{j}^{\prime}=1$. Pour tout $n \in \mathbb{N}$, on peut alors écrire

$$
\frac{\left(\alpha_{1}\right)_{n} \ldots\left(\alpha_{r}\right)_{n}}{n !\left(\beta_{1}\right)_{n} \ldots\left(\beta_{s}\right)_{n}}=\frac{\prod_{i=1}^{k_{0}} \prod_{\alpha \in R_{N_{i}}}(\alpha)_{n}}{\prod_{j=1}^{k_{0}^{\prime}} \prod_{\beta \in R_{N_{j}^{\prime}}}(\beta)_{n}}(1)_{n}^{k-k_{0}-\left(k^{\prime}-k_{0}^{\prime}\right)-1} .
$$


On a $C_{(\alpha, \beta)}=\frac{\prod_{i=1}^{k} C_{N_{i}}}{\prod_{j=1}^{k^{\prime}} C_{N_{j}^{\prime}}}=\frac{\prod_{i=1}^{k_{0}} C_{N_{i}}}{\prod_{j=1}^{k_{0}^{\prime}} C_{N_{j}^{\prime}}}$, car $C_{1}=1$. Ainsi, en multipliant (6.3) par $C_{(\alpha, \beta)}^{n}$, pour tout $n \in \mathbb{N}$, on obtient

$$
\begin{aligned}
C_{(\alpha, \beta)}^{n} & \frac{\left(\alpha_{1}\right)_{n} \cdots\left(\alpha_{r}\right)_{n}}{n !\left(\beta_{1}\right)_{n} \cdots\left(\beta_{s}\right)_{n}}= \\
& \left(\prod_{i=1}^{k_{0}} C_{N_{i}}^{n} \frac{\prod_{\alpha \in R_{N_{i}}}(\alpha)_{n}}{(n !)^{\varphi\left(N_{i}\right)}}\right)\left(\prod_{j=1}^{k_{0}^{\prime}} \frac{1}{C_{N_{j}^{\prime}}^{n}} \frac{(n !)^{\varphi\left(N_{j}^{\prime}\right)}}{\prod_{\beta \in R_{N_{j}^{\prime}}}(\beta)_{n}}\right)(n !)^{\sum_{i=1}^{k_{0}} \varphi\left(N_{i}\right)-\sum_{j=1}^{k_{0}^{\prime}} \varphi\left(N_{j}^{\prime}\right)}(1)_{n}^{k-k_{0}-\left(k^{\prime}-k_{0}^{\prime}\right)-1} .
\end{aligned}
$$

On a $(1)_{n}^{k-k_{0}-\left(k^{\prime}-k_{0}^{\prime}\right)-1}=n !^{k-k_{0}-\left(k^{\prime}-k_{0}^{\prime}\right)-1}=n !^{\sum_{i=k_{0}+1}^{k} \varphi\left(N_{i}\right)-\sum_{j=k_{0}^{\prime}+1}^{k^{\prime}} \varphi\left(N_{j}^{\prime}\right)-1}$, car, pour tout $i \in\left\{k_{0}+\right.$ $1, \ldots, k\}$ et tout $j \in\left\{k_{0}^{\prime}+1, \ldots, k^{\prime}\right\}$, on a $\varphi\left(N_{i}\right)=\varphi\left(N_{j}^{\prime}\right)=\varphi(1)=1$. Ainsi, d'après le lemme [17, il existe des entiers strictement positifs $e_{1}, \ldots, e_{q_{1}}, f_{1}, \ldots, f_{q_{2}}$ tels que

$$
C_{(\alpha, \beta)}^{n} \frac{\left(\alpha_{1}\right)_{n} \cdots\left(\alpha_{r}\right)_{n}}{n !\left(\beta_{1}\right)_{n} \cdots\left(\beta_{s}\right)_{n}}=\frac{\left(e_{1} n\right) ! \cdots\left(e_{q_{1}} n\right) !}{\left(f_{1} n\right) ! \cdots\left(f_{q_{2}} n\right) !}(n !)^{\sum_{i=1}^{k} \varphi\left(N_{i}\right)-\sum_{j=1}^{k^{\prime}} \varphi\left(N_{j}^{\prime}\right)-1} .
$$

D'où le fait que $P \subset Q$.

Montrons l'inclusion inverse $Q \subset P$.

Pour tout $\ell \in\left\{1, \ldots, e_{1}\right\}$, on a

$$
\left(\frac{\ell}{e_{1}}\right)_{n}=\frac{\ell}{e_{1}}\left(\frac{\ell}{e_{1}}+1\right) \cdots\left(\frac{\ell}{e_{1}}+n-1\right)=\frac{\ell}{e_{1}} \frac{\ell+e_{1}}{e_{1}} \cdots \frac{\ell+(n-1) e_{1}}{e_{1}} .
$$

Les numérateurs de (6.4) correspondent aux nombres dans $\left\{1, \ldots, n e_{1}\right\}$ qui sont congrus à $\ell$ modulo $e_{1}$. Ainsi, si $e_{1} \geq 2$, alors on a

$$
\left(e_{1} n\right) !=\left(\frac{1}{e_{1}}\right)_{n}\left(\frac{2}{e_{1}}\right)_{n} \ldots\left(\frac{e_{1}}{e_{1}}\right)_{n} e_{1}^{e_{1} n}=\left(\frac{1}{e_{1}}\right)_{n} \ldots\left(\frac{e_{1}-1}{e_{1}}\right)_{n}(1)_{n}\left(e_{1}^{e_{1}}\right)^{n}
$$

et si $e_{1}=1$, alors on a simplement $n !=(1)_{n}$. On peut donc écrire

$$
\frac{\left(e_{1} n\right) ! \cdots\left(e_{q_{1}} n\right) !}{\left(f_{1} n\right) ! \cdots\left(f_{q_{2}} n\right) !}=C^{n} \frac{\prod_{\substack{1 \leq i \leq q_{1} \\ e_{i} \geq 2}}\left(\left(\frac{1}{e_{i}}\right)_{n} \cdots\left(\frac{e_{i}-1}{e_{i}}\right)_{n}\right)}{n ! \prod_{\substack{1 \leq j \leq q_{2} \\ f_{j} \geq 2}}\left(\left(\frac{1}{f_{j}}\right)_{n} \cdots\left(\frac{f_{j}-1}{f_{j}}\right)_{n}\right)}(1)_{n}^{q_{1}-q_{2}+1}
$$

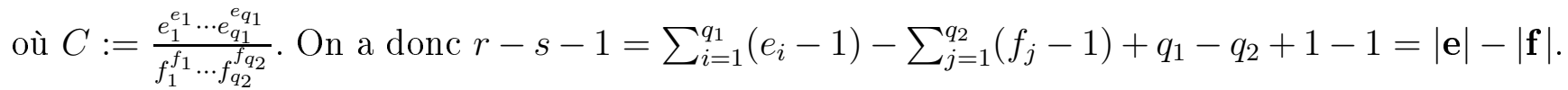

Si $e_{1} \geq 2$, alors on a

$$
\left\{\frac{1}{e_{1}}, \ldots, \frac{e_{1}-1}{e_{1}}\right\}=\bigcup_{d \mid e_{1}, d \geq 2} R_{d}
$$

En effet, notons $e_{1}=p_{1}^{a_{1}} \cdots p_{s}^{a_{s}}$ la décomposition en facteurs premiers de $e_{1}$. On a

$$
\left\{1, \ldots, e_{1}-1\right\}=\bigcup_{\substack{0 \leq b_{1} \leq a_{1}, \ldots, 0 \leq b_{s} \leq a_{s} \\\left(b_{1}, \ldots, b_{s}\right) \neq\left(a_{1}, \ldots, a_{s}\right)}}\left\{p_{1}^{b_{1}} \cdots p_{s}^{b_{s}} r:\left(r, p_{1} \cdots p_{s}\right)=1,1 \leq r<p_{1}^{a_{1}-b_{1}} \cdots p_{s}^{a_{s}-b_{s}}\right\} .
$$


Ainsi, on obtient

$$
\begin{aligned}
\left\{\frac{1}{e_{1}}, \ldots, \frac{e_{1}-1}{e_{1}}\right\} & =\bigcup_{\substack{0 \leq b_{1} \leq a_{1}, \ldots, 0 \leq b_{s} \leq a_{s} \\
\left(b_{1}, \ldots, b_{s}\right) \neq\left(a_{1}, \ldots, a_{s}\right)}}\left\{\frac{r}{p_{1}^{a_{1}-b_{1}} \cdots p_{s}^{a_{s}-b_{s}}}:\left(r, p_{1} \cdots p_{s}\right)=1, r<p_{1}^{a_{1}-b_{1}} \cdots p_{s}^{a_{s}-b_{s}}\right\} \\
& =\bigcup_{\substack{d \mid e_{1}, d \geq 2 \\
\bigcup_{d} .}} R_{d} .
\end{aligned}
$$

En utilisant l'identité (6.6) dans (6.5), on obtient

$$
\frac{\left(e_{1} n\right) ! \cdots\left(e_{q_{1}} n\right) !}{\left(f_{1} n\right) ! \cdots\left(f_{q_{2}} n\right) !}=C^{n} \frac{\prod_{i=1}^{q_{1}} \prod_{\substack{d \mid e_{i} \\ d \geq 2}} \prod_{\alpha \in R_{d}}(\alpha)_{n}}{n ! \prod_{j=1}^{q_{2}} \prod_{\substack{d \mid f_{j} \\ d \geq 2}} \prod_{\beta \in R_{d}}(\beta)_{n}}\left(\prod_{\gamma \in R_{1}}(\gamma)_{n}\right)^{q_{1}-q_{2}+1}
$$

où ici $\prod_{\gamma \in R_{1}}(\gamma)_{n}=(1)_{n}$. Si $q_{1}-q_{2}+1$ est positif alors on regroupe le produit des $\gamma$ avec celui des $\alpha$ au numérateur, et si $q_{1}-q_{2}+1$ est négatif alors on regroupe le produit des $\gamma$ avec celui des $\beta$ au dénominateur. On indexe les $\alpha$ et $\beta$ afin d'obtenir l'écriture

$$
\frac{\left(e_{1} n\right) ! \cdots\left(e_{q_{1}} n\right) !}{\left(f_{1} n\right) ! \cdots\left(f_{q_{2}} n\right) !}=C^{n} \frac{\left(\alpha_{1}\right)_{n} \cdots\left(\alpha_{r}\right)_{n}}{n !\left(\beta_{1}\right)_{n} \cdots\left(\beta_{s}\right)_{n}},
$$

où les suites $\alpha:=\left(\alpha_{i}\right)_{i=1, \ldots, r}$ et $\beta:=\left(\beta_{j}\right)_{j=1, \ldots, s}$ sont $R$-partitionnées.

Il ne reste plus qu'à montrer que $C=C_{(\alpha, \beta)}$. Comme $P \subset Q$, il existe des entiers strictement positifs $e_{1}^{\prime}, \ldots, e_{q_{1}^{\prime}}^{\prime}, f_{1}^{\prime}, \ldots, f_{q_{2}^{\prime}}^{\prime}$ tels que, pour tout $n \in \mathbb{N}$, on ait

$$
C_{(\alpha, \beta)}^{n} \frac{\left(\alpha_{1}\right)_{n} \cdots\left(\alpha_{r}\right)_{n}}{n !\left(\beta_{1}\right)_{n} \cdots\left(\beta_{s}\right)_{n}}=\frac{\left(e_{1}^{\prime} n\right) ! \cdots\left(e_{q_{1}^{\prime}}^{\prime} n\right) !}{\left(f_{1}^{\prime} n\right) ! \cdots\left(f_{q_{2}^{\prime}}^{\prime} n\right) !} .
$$

En divisant le terme de gauche de l'égalité (6.7) par le terme de droite de l'égalité (6.8), on obtient, pour tout $n \in \mathbb{N}$,

$$
\frac{\left(e_{1} n\right) ! \cdots\left(e_{q_{1}} n\right) !\left(f_{1}^{\prime} n\right) ! \cdots\left(f_{q_{2}^{\prime}}^{\prime} n\right) !}{\left(f_{1} n\right) ! \cdots\left(f_{q_{2}} n\right) !\left(e_{1}^{\prime} n\right) ! \cdots\left(e_{q_{1}^{\prime}}^{\prime} n\right) !}=\left(\frac{C}{C_{(\alpha, \beta)}}\right)^{n} .
$$

Raisonnons par l'absurde et supposons que $C$ soit différent de $C_{(\alpha, \beta)}$. Il existe deux suites d'entiers strictement positifs disjointes $\left(c_{1}, \ldots, c_{k}\right)$ et $\left(d_{1}, \ldots, d_{\ell}\right)$ telles que, pour tout $n \in \mathbb{N}$, on ait

$$
\frac{\left(e_{1} n\right) ! \cdots\left(e_{q_{1}} n\right) !\left(f_{1}^{\prime} n\right) ! \cdots\left(f_{q_{2}^{\prime}}^{\prime} n\right) !}{\left(f_{1} n\right) ! \cdots\left(f_{q_{2}} n\right) !\left(e_{1}^{\prime} n\right) ! \cdots\left(e_{q_{1}^{\prime}}^{\prime} n\right) !}=\frac{\left(c_{1} n\right) ! \cdots\left(c_{k} n\right) !}{\left(d_{1} n\right) ! \cdots\left(d_{\ell} n\right) !} .
$$

Comme $C / C_{(\alpha, \beta)} \neq 1$ et d'après l'identité (6.9),$\left(c_{1}, \ldots, c_{k}\right)$ et $\left(d_{1}, \ldots, d_{\ell}\right)$ ne peuvent être simultanément vides. Soit $M:=\max \left(c_{1}, \ldots, c_{k}, d_{1}, \ldots, d_{\ell}\right)$. D'après (6.5) et par unicité de l'écriture sous forme de coefficients hypergéométriques i.e. proposition 1, le symbole de Pochhammer $(1 / M)_{n}$ devrait apparaître dans le terme de droite de l'égalité (6.9), mais ce n'est pas le cas. D'où la contradiction.

6.2. Description des sauts de l'application $\Delta$ de Landau. Dans cette partie, nous allons montrer que les abscisses et les amplitudes des sauts effectués par $\Delta_{(\mathbf{e}, \mathbf{f})}$ sur $[0,1]$ apparaissent naturellement dans la réécriture de $\mathcal{Q}_{(\mathbf{e}, \mathbf{f})}$ sous la forme $\mathcal{P}_{(\alpha, \beta)}$.

Proposition 3. Soit $\boldsymbol{e}$ et $\boldsymbol{f}$ deux suites finies d'entiers strictement positifs. On peut écrire de manière unique la suite $\mathcal{Q}_{(\boldsymbol{e}, \boldsymbol{f})}$ sous la forme

$$
\mathcal{Q}_{(\boldsymbol{e}, \boldsymbol{f})}(n)=C^{n}\left(\gamma_{1}\right)_{n}^{m_{1}} \ldots\left(\gamma_{t}\right)_{n}^{m_{t}}, n \geq 0
$$


où $C$ est une constante strictement positive, $0<\gamma_{1}<\cdots<\gamma_{t} \leq 1$ sont des rationnels et les $m_{1}, \ldots, m_{t}$ sont dans $\mathbb{Z} \backslash\{0\}$. Les sauts de $\Delta_{(\boldsymbol{e}, \boldsymbol{f})}$ sur $[0,1]$ se font aux abscisses $\gamma_{1}, \ldots, \gamma_{t}$ avec $m_{i}$ pour amplitude (positive ou négative) en $\gamma_{i}$.

Démonstration. On écrit $\mathbf{e}:=\left(e_{1}, \ldots, e_{q_{1}}\right)$ et $\mathbf{f}:=\left(f_{1}, \ldots, f_{q_{2}}\right)$. L'existence et l'unicité de l'écriture (6.10) découlent respectivement des propositions 2 et 1 de la partie 6.1, Étudions les sauts de la fonction $\Delta_{(\mathbf{e}, \mathbf{f})}$.

Si $c \in \mathbb{N}, c \geq 1$, alors la fonction $[0,1] \longrightarrow \mathbb{Z}, x \longmapsto\lfloor c x\rfloor$ effectue un saut d'amplitude 1 en $\frac{1}{c}, \frac{2}{c}, \ldots, \frac{c-1}{c}$ et 1 . D'après (6.5), pour tout $n \geq 0$, on a

$$
\mathcal{Q}_{(\mathbf{e}, \mathbf{f})}(n)=C^{n} \frac{\left(\frac{1}{e_{1}}\right)_{n} \ldots\left(\frac{e_{1}-1}{e_{1}}\right)_{n} \ldots\left(\frac{1}{e_{q_{1}}}\right)_{n} \ldots\left(\frac{e_{q_{1}}-1}{e_{q_{1}}}\right)_{n}}{\left(\frac{1}{f_{1}}\right)_{n} \ldots\left(\frac{f_{1}-1}{f_{1}}\right)_{n} \ldots\left(\frac{1}{f_{q_{2}}}\right)_{n} \ldots\left(\frac{f_{q_{2}}-1}{f_{q_{2}}}\right)_{n}}(1)_{n}^{q_{1}-q_{2}} .
$$

Ainsi, en simplifiant le quotient et en regroupant les symboles de Pochhammer identiques, on obtient l'écriture $\mathcal{Q}_{(\mathbf{e}, \mathbf{f})}(n)=C^{n}\left(\gamma_{1}\right)_{n}^{m_{1}} \ldots\left(\gamma_{t}\right)_{n}^{m_{t}}, n \geq 0$, où les $\gamma_{1}<\cdots<\gamma_{t}$ correspondent effectivement aux abscisses des sauts de $\Delta_{(\mathbf{e}, \mathbf{f})}$ et les $m_{i}, 1 \leq i \leq t$, à leur amplitude.

Remarque. Des résultats analogues à ceux de la proposition [3 sont mentionnés dans [1] par Rodriguez-Villegas puis précisés dans [2] par Bober.

Supposons que, pour tout $x \in[0,1]$, on ait $\Delta_{(\mathbf{e}, \mathbf{f})}(x) \geq 0$. Alors, comme $\Delta_{(\mathbf{e}, \mathbf{f})}(0)=\Delta_{(\mathbf{e}, \mathbf{f})}\left(\gamma_{t}\right)=$ $0, \gamma_{1}$ correspond à un saut d'amplitude positive et $\gamma_{t}$ correspond à un saut d'amplitude négative. On remarque grâce à l'identité (6.5) que $\gamma_{1}=1 / M_{(\mathbf{e}, \mathbf{f})}$ et $\gamma_{t}=\left(M_{(\mathbf{e}, \mathbf{f})}-1\right) / M_{(\mathbf{e}, \mathbf{f})}$ ou 1 , où $M_{(\mathbf{e}, \mathbf{f})}=\max \left(e_{1}, \ldots, e_{q_{1}}, f_{1}, \ldots, f_{q_{2}}\right)$. Or $\gamma_{1}$ correspond à un saut d'amplitude positive donc $M_{(\mathbf{e}, \mathbf{f})}=\max \left(e_{1}, \ldots, e_{q_{1}}\right):=M_{\mathbf{e}}$ et $\gamma_{t}=1$ car $\left(M_{\mathbf{e}}-1\right) / M_{\mathbf{e}}$ correspond aussi à l'abscisse d'un saut d'amplitude positive. En résumé, on a $\gamma_{1}=1 / M_{\mathbf{e}}, \gamma_{t}=1$ et, pour tout $x \in\left[\left(M_{\mathbf{e}}-1\right) / M_{\mathbf{e}}, 1[\right.$, on a $\Delta_{(\mathbf{e}, \mathbf{f})}(x) \geq 1$.

Supposons de plus que, pour tout $x \in\left[1 / M_{\mathbf{e}}, 1\left[\right.\right.$, on ait $\Delta_{(\mathbf{e}, \mathbf{f})}(x) \geq 1$. On sait alors que $\left(M_{\mathbf{e}}-\right.$ 1) $/ M_{\mathbf{e}}$ correspond à l'abscisse d'un saut d'amplitude positive.

Si $M_{\mathbf{e}} \geq 3$, alors on a $\left(M_{\mathbf{e}}-1\right) / M_{\mathbf{e}}>1 / M_{\mathbf{e}}$ et, pour tout $x \in\left[1 / M_{\mathbf{e}} ;\left(M_{\mathbf{e}}-1\right) / M_{\mathbf{e}}[\right.$ on a $\Delta_{(\mathbf{e}, \mathbf{f})}(x) \geq 1$, donc pour tout $x \in\left[\left(M_{\mathbf{e}}-1\right) / M_{\mathbf{e}}, 1\left[\right.\right.$ on a $\Delta_{(\mathbf{e}, \mathbf{f})}(x) \geq 2$. Ainsi $m_{t} \leq-2$, ce qui signifie que dans l'écriture des coefficients hypergéométriques sous la forme $C_{(\alpha, \beta)}^{n} \frac{\left(\alpha_{1}\right)_{n} \ldots\left(\alpha_{r}\right)_{n}}{(1)_{n}\left(\beta_{1}\right)_{n} \ldots\left(\beta_{r-1}\right)_{n}}$, au moins un des $\beta_{i}$ est égal à 1. D'après [3, p. 310], ceci implique que l'équation différentielle fuchsienne associée à $F_{(\mathbf{e}, \mathbf{f})}$ admet une solution de type logarithmique à l'origine $G_{(\mathbf{e}, \mathbf{f})}(z)+\log (z) F_{(\mathbf{e}, \mathbf{f})}(z)$, où $G_{(\mathbf{e}, \mathbf{f})}$ est définie par l'identité (1.1) donnée dans la partie 1.1 .

Si $M_{\mathbf{e}}=2$, alors $\mathcal{Q}_{(\mathbf{e}, \mathbf{f})}(n)$ est de la forme $\mathcal{Q}_{(\mathbf{e}, \mathbf{f})}(n)=\frac{(2 n) ! j}{n !^{j j}}=\left(2^{2 j}\right)^{n} \frac{(1 / 2)_{n}^{j}}{(1)_{n}^{j}}$, où $j \in \mathbb{N}, j \geq 1$. Ainsi le seul couple de suites $(\mathbf{e}, \mathbf{f})$ dont l'équation différentielle hypergéométrique associée n'admet pas de solution de type logarithmique à l'origine correspond au cas $j=1$.

Nous allons maintenant montrer que si e et $\mathbf{f}$ sont deux suites d'entiers strictement positifs disjointes vérifiant $|\mathbf{e}|=|\mathbf{f}|$, alors les assertions suivantes sont équivalentes.

(i) $\Delta_{(\mathbf{e}, \mathbf{f})}$ est croissante sur $[0,1[$.

(ii) Il existe des entiers strictement positifs $N_{1}, \ldots, N_{k}$ tels que e est constituée des éléments du multi-ensemble $\bigcup_{i=1}^{k} A_{N_{i}}$ et $\mathbf{f}$ est constituée des éléments du multi-ensemble $\bigcup_{i=1}^{k} B_{N_{i}}$, où les multi-ensembles $A_{N_{i}}$ et $B_{N_{i}}$ sont définis comme dans la partie 1.2.

(iii) L'équation différentielle fuchsienne associée à $F_{(\mathbf{e}, \mathbf{f})}$ a tous ses exposants égaux à 0 à l'origine.

Soit $\left(\alpha_{1}, \ldots, \alpha_{r}\right)$ et $\left(\beta_{1}, \ldots, \beta_{s}\right)$ les suites telles que $\mathcal{Q}_{(\mathbf{e}, \mathbf{f})}=\mathcal{P}_{(\alpha, \beta)}$. 
Si $\Delta_{(\mathbf{e}, \mathbf{f})}$ est croissante sur $\left[0,1\left[\right.\right.$ alors $\beta_{1}=\cdots=\beta_{s}=1$ et l'équation différentielle fuchsienne associée à $F_{(\mathbf{e}, \mathbf{f})}$ a donc tous ses exposants égaux à 0 à l'origine (voir [12]). Ainsi, on a $(i) \Rightarrow(i i i)$. De plus, comme $\alpha$ est $R$-partitionnée, il existe des entiers strictement positifs $N_{1}, \ldots, N_{k}$ tels que $\left\{\alpha_{1}, \ldots, \alpha_{r}\right\}=\bigcup_{i=1}^{k} R_{N_{i}}$. Ainsi, d'après le lemme 17, on obtient bien $(i) \Rightarrow(i i)$.

Réciproquement, s'il existe des entiers strictement positifs $N_{1}, \ldots, N_{k}$ tels que e est constituée des éléments du multi-ensemble $\bigcup_{i=1}^{k} A_{N_{i}}$ et $\mathbf{f}$ est constituée des éléments du multi-ensemble $\bigcup_{i=1}^{k} B_{N_{i}}$ alors le lemme 17 montre que $\beta_{1}=\cdots=\beta_{s}=1$. On a donc bien $(i) \Leftrightarrow(i i)$.

Si l'équation différentielle fuchsienne associée à $F_{(\mathbf{e}, \mathbf{f})}$ a tous ses exposants égaux à 0 à l'origine, alors $\beta_{1}=\cdots=\beta_{s}=1$ et $\Delta_{(\mathbf{e}, \mathbf{f})}$ est croissante sur $[0,1[$. On obtient bien $(i) \Leftrightarrow(i i i)$.

\section{RÉFÉRENCES}

[1] V. V. Batyrev and D. van Straten, Generalized hypergeometric functions and rational curves on Calabi-Yau complete intersections in toric varieties, Comm. Math. Phys. 168 (1995), 493-533.

[2] J. W. Bober, Factorial ratios, hypergeometric series, and a family of step functions, to appear in the journal of the London Mathematical Society. arXiv :math.NT/0709.1977v1.

[3] B. Dwork, On p-adic differential equations IV generalized hypergeometric functions as p-adic functions in one variable, Annales scientifiques de l'E.N.S. $4^{e}$ série, tome 6, numero 3 (1973), p. 295-316.

[4] N. Kobliz, p-Adic Numbers, p-Adic Analysis, and Zeta-functions, Springer-Verlag, Heidelberg, 1977.

[5] C. Krattenthaler and T. Rivoal, On the integrality of the Taylor coefficients of mirror maps, 36 pages, à paraitre à Duke Math. J.

[6] E. Landau, Sur les conditions de divisibilité d'un produit de factorielles par un autre, collected works, I, page 116. Thales-Verlag, 1985.

[7] S. Lang, Cyclotomic Fields, I, II, Combined 2nd edition, vol. 121, Graduate Texts in Math., Springer-Verlag, New York, 1990.

[8] B. H. Lian, S. T. Yau, Mirror Maps, Modular Relations and Hypergeometric Series I. arXiv :hep-th/9507151v1. Paru sous le titre : Integrality of certain exponential series. Algebra and geometry (Taipei, 1995), 215-227, Lect. Algebra Geom., 2, Int. Press, Cambridge, MA, 1998. (Reviewer : Nobuo Tsuzuki).

[9] B. H. Lian, S. T. Yau, Arithmetic properties of mirror map and quantum coupling, Comm. Math. Phys. 176, no. 1 (1996), 163-191.

[10] K. Ota, On special values of generalized p-adic hypergeometric functions. Acta Arith. 67 (1994), no. 2, 141-163.

[11] F. Rodriguez-Villegas, Integral ratios of factorials and algebraic hypergeometric functions, in : Oberwolfach Reports, vol. 2, issue 3, European Math. Soc., Publ. House, Zürich, 2005; disponible sur http://www.math.utexas.edu/villegas/publications/oberwolfach-05.pdf.

[12] M. Yoshida, Fuchsian differential equations, Aspects of Mathematics 11, Vieweg, 1987.

[13] V. V. Zudilin, Integrality of power expansions related to hypergeometric series, Mathematical Notes, vol. 71, no. 5, 2002, p. 604-616.

E. Delaygue, Institut Fourier, CNRS et Université Grenoble 1, 100 rue des Maths, BP 74, 38402 Saint-Martin-d'Hères cedex, France. Email : Eric.Delaygue@ujf-grenoble.fr 